\title{
Detection of juxta-pleural lung nodules in computed tomography images
}

\author{
M.Sc. Dissertation
}

Guilherme Moreira Aresta

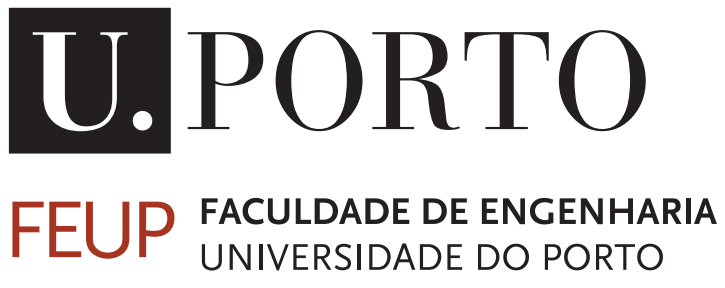

Mestrado Integrado em Bioengenharia

Supervisor: Aurélio Campilho, Full Professor, FEUP

Co-supervisor: António Cunha, Assistant Professor, UTAD 

Detection of juxta-pleural lung nodules in computed tomography images

\section{M.Sc. Dissertation}

Guilherme Moreira Aresta

Mestrado Integrado em Bioengenharia

June 23, 2016 



\section{Resumo}

O cancro é uma patologia caracterizada por uma divisão anormal das células e posterior metástase para outros tecidos. Esta doença é uma das principais causas de morte a nível mundial. Dos diferentes tipos de cancro, o do pulmão é o mais letal. Contudo, o diagnóstico precoce aumenta a taxa de sobrevivência dos pacientes. O diagnóstico pode ser feito através da detecção de nódulos em imagens de tomografia axial computorizada. No entanto, o cansaço, distração e limitações do sistema visual humano reduzem o sucesso da detecção e consequentemente do diagnóstico da patologia pelos especialistas. Por isso, o sucesso do diagnóstico pode ser melhorado através de sistemas de detecção assistida por computador. Sabe-se que sistemas dedicados a um determinado tipo de nódulo têm melhores resultados do que abordagens mais genéricas. Dos diferentes tipos de nódulos, os justapleurais são aqueles com piores resultados de detecção.

Nesta dissertação propõe-se um método dedicado à deteção de nódulos justapleurais. O nosso foco são nódulos com raio $\leq 5 \mathrm{~mm}$ uma vez que estes são os mais difíceis de detectar. A abordagem proposta explora a diferença de intensidade entre os nódulos e o parênquima do pulmão, bem como a sua estrutura naturalmente arredondada. Os potenciais candidatos são pesquisados dentro de uma segmentação do volume do pulmão obtida através de uma técnica de crescimento de regiões com base em intensidade. De forma a incluir os nódulos justapleurais, os contornos são refinados usando operações morfológicas e contornos activos. Os nódulos sólidos são detectados através de uma saturação local da intensidade e da selecção de um limiar de segmetação apropriado. Os nódulos não-sólidos e sub-sólidos, por sua vez, são realçados através de uma filtragem muli-escala com Laplacianos-do-Gaussiano. Finalmente, os resultados das duas abordagens são combinados para produzir uma lista de candidatos. Os falsos positivos mais óbvios desta lista são eliminados através de regras fixas relacionadas com o diâmetro dos candidatos. De seguida, características associadas com a intensidade, redondeza, geometria e localização dos candidatos são usadas para treinar uma máquina de vectores de suporte e consequentemente reduzir o número de falsos positivos. O nosso método foi desenvolvido e avaliado na "Lung Image Database Consortium image collection". Como esta base de dados não caracteriza os nódulos de acordo com a sua posição, é também proposto um sub-conjunto apenas com nódulos justapleurais.

O sistema apresentado para detecção de nódulos justapleurais tem resultados equiparáveis ou melhores do que os métodos do estado da arte. A sensibilidade do nosso método é de $57 \%$ com 4 falsos positivos por exame, aumentando para $62 \%$ se apenas forem considerados nódulos sólidos. 


\section{Abstract}

Cancer is characterized by an abnormal cell division and spread of cells to other tissues. This condition is a leading cause of death worldwide. From the different cancer types, lung cancer is the most lethal. Survival rate is known to increase if the diagnosis is performed early, which can be achieved by analyzing computed tomography scans. However, fatigue, distraction and limitations of the human visual system reduce the overall detection and diagnosis success. Computer-aided detection systems can improve nodule detection rate by providing a second opinion to the physicians. Computer-aided detection systems dedicated to specific kinds of nodules tend to outperform general approaches. Nodules can be classified according to their location as isolated, peri-fissural, juxta-vascular and juxta-pleural. From these types of nodules, computer-aided detection systems operate worse for juxta-pleural nodules.

A dedicated juxta-pleural lung nodule detection method is proposed. We focus on nodules with radius $\leq 5 \mathrm{~mm}$ because they are more challenging to detect. The proposed approach exploits the intensity difference between the majority of the nodules and the parenchymal tissue, as well as the partial roundness of these structures. Lung nodule candidates are searched inside a regiongrowing based lung volume segmentation that is refined with morphological operations and active contours to include juxta-pleural nodules. First, solid nodules are detected with a local contrast saturation followed by the selection of an appropriate threshold over a sliding window. Sub-solid and non-solid nodules are enhanced using multi-scale Laplacian-of-Gaussian filtering. The results from the two approaches are then combined to produce a single candidate list. The number of false positives is reduced using fixed-rules related to the radius of the candidates. Then, intensity, blobness, geometric and location features are extracted and used to train a Support Vector Machine classifier with radial basis function to further reduce the number of false positive detections. The method is developed and tested using the Lung Image Database Consortium image collection. This dataset does not characterize nodules location-wise and thus a sub-dataset with the juxta-pleural lung nodules is also proposed.

The proposed framework achieves similar or better juxta-pleural lung nodule detection performance in comparison to state-of-the-art methods. The achieved sensitivity is $57 \%$ with 4 false positives per scan, increasing to $62 \%$ if only solid nodules are considered. 


\section{Acknowledgements}

This work is financed by the ERDF - European Regional Development Fund through the Operational Programme for Competitiveness and Internationalisation - COMPETE 2020 Programme, and by National Funds through the FCT - Fundação para a Ciência e a Tecnologia within project POCI-01-0145-FEDER-016673

\section{C\$MPETE 2020

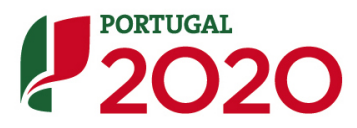

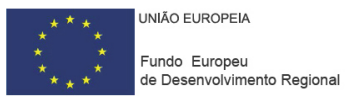

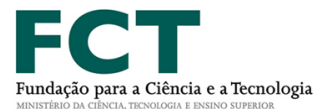




\section{Contents}

Preamble 1

Motivation, objectives and contributions .............. 1

M.Sc. dissertation overview . . . . . . . . . . . . . . . . 2

1 Introduction 3

1.1 Anatomical context ...................... 3

1.2 Lung cancer and computed tomography $\ldots \ldots \ldots \ldots$

1.3 Lung nodule computer-aided detection systems $\ldots \ldots \ldots \ldots$ 5 . . . . 5

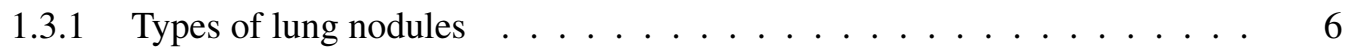

1.3.2 The LIDC/IDRI dataset . . . . . . . . . . . . . . . . . . . . 7

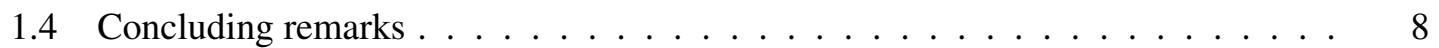

2 Methods for lung nodule detection in CT images 9

2.1 Lung nodule detection steps . . . . . . . . . . . . . . . . . . 9 9

2.1.1 Candidate detection . . . . . . . . . . . . . . . . . 10

2.1 .2 Candidate refinement . . . . . . . . . . . . . . . 12

2.1.3 False positive reduction $\ldots \ldots \ldots \ldots \ldots$

2.2 Representative lung nodule detection algorithms . . . . . . . . . . . . . 15

2.2.1 Juxta-pleural nodules specific approaches . . . . . . . . . . . . . . 16

2.3 Results review . . . . . . . . . . . . . . . . . . . 17

2.3.1 Detection per nodule type . . . . . . . . . . . . . . 19

2.4 Concluding remarks . . . . . . . . . . . . . . . . . . . . . . 21

3 Juxta-pleural lung nodule detection 23

3.1 Method overview . . . . . . . . . . . . . . . . . . 23

3.2 Lung volume segmentation . . . . . . . . . . . . . . . . . . 24

3.2.1 Initial lung volume segmentation . . . . . . . . . . . . . . 24

3.2 .2 Active contour refinement . . . . . . . . . . . . . 25

3.3 Dataset of juxta-pleural lung nodules . . . . . . . . . . . . . . 26

3.4 Candidate detection . . . . . . . . . . . . . . . . . 27

3.4 .1 Direct threshold . . . . . . . . . . . . . . . . . . . . 27

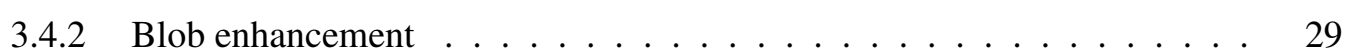

3.5 False positive reduction . . . . . . . . . . . . . . . 30

3.5.1 Fixed-rules based FP reduction . . . . . . . . . . . . . . . . . . 30

3.5.2 Supervised learning FP reduction . . . . . . . . . . . . . . 30

3.6 Performance evaluation . . . . . . . . . . . . . . . . . . 33

3.7 Concluding remarks . . . . . . . . . . . . . . . . . . 34 
4 Results and discussion $\quad 35$

4.1 Dataset of juxta-pleural nodules . . . . . . . . . . . . . 35

4.2 Lung volume segmentation . . . . . . . . . . . . . . . . 38

4.3 Candidate detection . . . . . . . . . . . . . . 40

4.4 False positive reduction . . . . . . . . . . . . . . 43

4.4.1 Fixed-rules based FP reduction . . . . . . . . . . . . . . . . 43

4.4 .2 Supervised learning FP reduction . . . . . . . . . . . . . . . 44

4.5 Comparison with existing methods . . . . . . . . . . . 45

4.6 Concluding remarks . . . . . . . . . . . . . . . . . . . . . 48

5 Conclusion and Future work 49

A Juxta-pleural lung nodules

References 


\section{List of Figures}

1.1 Schematic representation of the anatomy of the lungs. Adapted from OpenStaxCollege-

Anatomy\&Physiology (2013). . . . . . . . . . . . . .

1.2 Hounsfield unit values for different types of tissue and materials commonly present in a CT scan. -1000 corresponds to black and +1000 to white. Image from Holmes

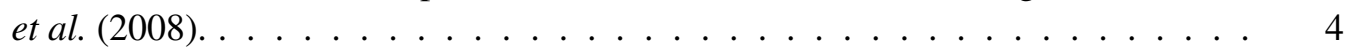

1.3 Example of a slice from a CT scan. Relevant structures (blood vessels, bone, fissures and lung parenchyma) are depicted. CT scan image from TheCancerImagingArchiveTeam . . . . . . . . . . . . . . 5

1.4 Nodule types according to opacity. Images from TheCancerImagingArchiveTeam 6

1.5 Nodule types according to relative location to lung structures. Images from TheCancerImagingArchiveTeam . . . . . . . . . . . . . .

2.1 Overall structure of a lung nodule detection algorithm.

2.2 Steps for juxta-pleural lung nodule detection using $\alpha$-hulls. i) juxta-pleural lung nodule; ii) pleural surface; iii) $\alpha$-hull partially overlaying the pleural surface; iv) segmented nodule, resulting from the subtraction of the $\alpha$-hull to the pleural surface. 12

2.3 Steps for juxta-pleural lung nodule detection using directional-gradient concentration. i) juxta-pleural lung nodule; ii) pleural surface; iii) normals to the surface; iv) detection of the nodule as the region with higher surface normals overlap.

2.4 Commonly used classifiers for false positive reduction of nodule candidates. . . .

2.5 Sensitivity (\%) and respective number of false positives/scan for different methods. The minimum nodule diameter $(\mathrm{mm})$, range of slice thickness $(\mathrm{mm})$ and number of scans studied are also shown. Values retrieved from: 1) Alilou et al. (2014); 2) Brown et al. (2014); 3) Camarlinghi et al. (2012); 4) Cascio et al. (2012); 5) Choi and Choi (2013); 6) Choi and Choi (2014); 7) van Ginneken et al. (2015); 8) Golosio et al. (2009); 9) Guo and Li (2012); 10) Han et al. (2015); 11) Messay et al. (2010); 12) Opfer and Wiemker (2007); 13) Setio et al. (2015); 14) Han et al. (2015); 15) Setio et al. (2015); 16) Tan et al. (2011); Setio et al. (2015); 17) Tan et al. (2013); 18) Teramoto and Fujita(2013). . . . . . . . . . . . . . . .

2.6 Average sensitivity values of several rates of false positives/scan $\left(2^{-3}, 2^{-2}, \ldots, 2^{3}\right)$ for different type of nodules and algorithms. Data retrieved from van Ginneken et al. (2010) . . . . . . . . . . . . . . . . . . . . . . . 19

3.1 Schematic representation of the proposed method. . . . . . . . . . . . .

3.2 Relevant steps of the region growing-based algorithm for lung volume segmentation. The initial segmentation (prior to refinement) does not include nodules. Nodules are included after the segmentation is refined with a morphological closing. Blue scale bar corresponds to $5 \mathrm{~mm} . \ldots \ldots \ldots \ldots$. . . . . . . . 
3.3 Details of the correction of the lung volume segmentation via the Chan-Vese active contour method. The inclusion of juxta-pleural nodules is improved (c) and d). Blue scale bar corresponds to $5 \mathrm{~mm} . \ldots \ldots \ldots \ldots$

3.4 Examples of nodules considered as juxta-pleural. Each example is retrieved from a scan and slice (LIDC-IDRI case\#, slice\#). Blue scale bar corresponds to $5 \mathrm{~mm}$. .

3.5 Steps for juxta-pleural nodule detection. (a)-(d): solid nodule; (f) -(i): sub-solid nodule; ( $(\mathrm{k})$-(n): non-solid nodule. For the blob enhancement step, Laplacian-ofGaussian filters with $\sigma=\{1,1.5,2\}$ are used. Each example is retrieved from a scan and slice (LIDC-IDRI case\#, slice\#). Blue scale bar corresponds to $5 \mathrm{~mm}$. . .

3.6 Examples of Laplacian of Gaussian blob enhancement filters for different $\sigma$ values. 29

4.1 Examples of nodules removed from the juxta-pleural dataset. Each example is retrieved from a scan and slice (LIDC-IDRI case\#, slice\#). Blue scale bar corresponds to $5 \mathrm{~mm} \ldots \ldots \ldots \ldots \ldots \ldots \ldots \ldots \ldots$

4.2 Statistics retrieved from the LIDC-IDRI, with special focus on juxta-pleural nodules. Small juxta-pleural nodules have radius $\leq 5 \mathrm{~mm} \ldots \ldots \ldots \ldots \ldots$. . . . .

4.3 Examples of juxta-pleural lung nodules with radius $\leq 5 \mathrm{~mm}$ (first row) and nonnodule structures (second row). Each example is retrieved from a scan and slice (LIDC-IDRI case\#, slice\#). Blue scale bar corresponds to $5 \mathrm{~mm}$. . . . . . . . . . . .

4.4 Examples of nodules not included in the lung volume segmentation due to their location near the limits of the lungs. Each example is retrieved from a scan and slice (LIDC-IDRI case\#, slice\#). Blue scale bar corresponds to $5 \mathrm{~mm}$. . . . . . . . .

4.5 3D reconstruction of a juxta-pleural lung nodule candidate (orange) along with the ground-truth (yellow). The correspond initial, middle and final slices are shown. Each example is retrieved from a different slice (LIDC-IDRI case\#, slice\#). Blue scale bar corresponds to $5 \mathrm{~mm} . \ldots \ldots \ldots \ldots \ldots$

4.6 Juxta-pleural lung nodules with radius $\leq 5 \mathrm{~mm}$ (first row) and respective detection (second row). Each example is retrieved from a scan and slice (LIDC-IDRI case\#, slice\#). Blue scale bar corresponds to $5 \mathrm{~mm} . \ldots \ldots \ldots$. . . . . . . . . . .

4.7 Juxta-pleural lung nodule candidates marked with orange contour. The yellow contour corresponds to the ground-truth. Each example is retrieved from a scan and slice (LIDC-IDRI case\#, slice\#). Blue scale bar corresponds to $5 \mathrm{~mm}$. . . . .

4.8 ROC curves of the proposed classifier for the detection of juxta-pleural nodules with radius $\leq 5 \mathrm{~mm}$ after the false positive reduction. The standard deviation is

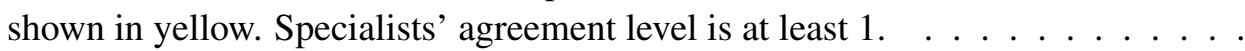

4.9 FROC curves of the proposed system for the detection of juxta-pleural nodules with radius $\leq 5 \mathrm{~mm}$ after the false positive reduction. Specialists' agreement level is at least 1 . 


\section{List of Tables}

$3.1 \quad$ Features used for supervised learning. . . . . . . . . . . . . . . . 31

4.1 Number of scans and juxta-pleural nodules in the studied 729 scans from the LIDC-IDRI dataset. Small juxta-pleural nodules are those with radius $\leq 5 \mathrm{~mm}$. . .

4.2 Inclusion of juxta-pleural nodules and juxta-pleural nodules with radius $\leq 5 \mathrm{~mm}$ by the segmented lung volume. . . . . . . . . . . . . . . .

4.3 Maximum achieved sensitivity values (\%) for the proposed method, prior to the false positive reduction step, per nodule texture and minimum agreement level between specialists. . . . . . . . . . . . . . . . . .

4.4 Maximum sensitivity values (\%) for juxta-pleural nodules reported by Retico et al. (2008); De Nunzio et al. (2011); Han et al. (2015) . . . . . . . . . . . . . . . . .

4.5 Maximum achieved sensitivity values (\%) for the proposed method, after the false positive reduction step, per nodule texture and minimum agreement level between specialists. . . . . . . . . . . . . . . . . . . .

4.6 Area under the ROC curve of the classifiers used to reduce the number of juxtapleural nodules reported by Retico et al. (2008); De Nunzio et al. (2011); Han et al. (2015). . . . . . . . . . . . . . . . . . 45

4.7 Performance of the proposed juxta-pleural lung nodule detection method for different nodule textures. . . . . . . . . . . . . . . . . . .

4.8 Juxta-pleural lung nodule detection performance of different systems. $r$ is the nodule radius. A - Retico et al. (2008), B - De Nunzio et al. (2011), C - Han et al. (2015), D - Fujitalab, E - Region growing volume plateau, F - Channeler Ant model, G - Voxel-based neural approach, H - ISI-CAD, I - Philips Lung Nodule CAD (van Ginneken et al. (2010)). . . . . . . . . . . . . . . . . . . 46

A.1 Juxta-pleural lung nodules available in the LIDC-IDRI dataset (1 out of 6) . . . 52 52

A.2 Juxta-pleural lung nodules available in the LIDC-IDRI dataset (2 out of 6) . . . . 53

A.3 Juxta-pleural lung nodules available in the LIDC-IDRI dataset (3 out of 6) . . . 5 54

A.4 Juxta-pleural lung nodules available in the LIDC-IDRI dataset (4 out of 6) . . . . 55

A.5 Juxta-pleural lung nodules available in the LIDC-IDRI dataset (5 out of 6) . . . 56

A.6 Juxta-pleural lung nodules available in the LIDC-IDRI dataset (6 out of 6) . . . 5 57 


\title{
Abbreviations and Symbols
}

\author{
2D Two-dimensional \\ 3D Three-dimensional \\ CADe Computer-aided detection \\ CADx Computer-aided diagnosis \\ CT Computed tomography \\ FF Feed-forward neural networks \\ FP False positive \\ FROC Free-response receiver operating characteristc \\ HU Hounsfield unit \\ LIDC/IDRI The Lung Image Database Consortium image collection \\ LoG Laplacian-of-Gaussian \\ SVM Support Vector Machine \\ ROC Receiver operating characteristc \\ TP True positive
}





\section{Preamble}

\section{Motivation, objectives and contributions}

Lung cancer is the most lethal type of cancer. CT is commonly used for detecting lung cancer. An early detection greatly increases the survival rate of the patients. However, fatigue and distraction errors, together with lack of experience and limitations of the visual system, cause physicians to fail during the nodule detection process. CADe systems are thus of interest, providing to the specialists a second opinion during their exam observation. The combination of CADe systems dedicated to a specific type of lung nodule is known to outperform more general approaches. The LIDC/IDRI is a lung CT public dataset used for training, testing and comparing the performance of CADe systems. Lung nodule CADe systems are usually composed by 3 steps: initial candidate detection, candidate refinement and FP reduction. Initial candidate detection is performed by using HU-based approaches, blob detectors or shape analysis. Candidates can be refined with region growing or active contours. The number of FPs can be reduced by using fixed rules followed by a binary classification with a trained classifier such as a SVM or a neural network.

Lung nodules can be classified according to their relative location to other lung anatomical structures as isolated, peri-fissural, juxta-vascular or juxta-pleural. Juxta-pleural nodules are lung lesions that occur near the pleura. These nodules have similar intensity to the pleural wall and their shape is more variable than other nodules. Considering these characteristics, the currently used approaches for automatic lung nodule detection in CT scans do not usually perform well for juxta-pleural nodule detection. In fact, these nodules tend to be the most difficult type of nodule to detect. Furthermore, there are few works addressing specifically the detection of juxta-pleural nodules in CT images. The following objective for the M.Sc. dissertation is thus proposed:

- development of a dedicated method for the detection of juxta-pleural nodules in lung CT images. The dedicated method overcomes several detection issues related to the characteristics of juxta-pleural nodules. The LIDC/IDRI dataset is used for the development of the algorithm. The proposed solution may be used for improving the performance of a multiapproach CADe system currently in development by the C-BER group.

The LIDC/IDRI dataset does not characterize lung nodules according to their location. Consequently, to validate our method, a sub-dataset with the juxta-pleural lung nodules existent in the LIDC/IDRI is also presented. 
Several contributions to the state-of-the-art result from this research:

- creation of a juxta-pleural lung nodule sub-dataset from the LIDC/IDRI dataset; to our knowledge this sub-dataset is the first to consider the majority of the scans of the LIDC/IDRI dataset and to be manually revised;

- improvement of the lung volume segmentation algorithm of the C-BER group in terms of juxta-pleural lung nodule inclusion;

- proposal of a juxta-pleural lung nodule detection method, with focus on nodules with radius $\leq 5 \mathrm{~mm}$; the method shows up-to state-of-the-art results and unlike other works considers all non-solid, sub-solid and solid nodules.

\section{M.Sc. dissertation overview}

The M.Sc. dissertation is organized as follows. Chapter 1 presents the anatomical context of the problem in study, along with the fundamentals of CT imaging and lung cancer detection. The characterization of lung nodules in terms of texture and location is also referred. Chapter 2 describes the state-of-the-art methods for the detection of lung nodules in CT scans, with focus on approaches dedicated to juxta-pleural lung nodules. Then, the overall and location-specific lung nodule detection performance of different systems is presented and discussed.

Chapter 3 presents our juxta-pleural lung nodule detection method. The method exploits the intensity difference between parenchymal tissue and lung nodules as well as the roundness of these abnormalities. Then, in Chapter 4, the performance of our system is discussed and compared to the state-of-the-art. Chapter 5 summarizes the main conclusions of the performed research and suggests future work to further improve the method. 


\section{Chapter 1}

\section{Introduction}

\subsection{Anatomical context}

The lungs (Fig. 1.1) are a set organs located within the thorax and are the most important organs of respiration. Each lung has an apex and a base (superior and inferior portion, respectively), three borders and two surfaces (costal and mediastinal). The right lung has 3 lobes and the left 2 lobes. The lungs are invested by a serous membrane called pleura, composed by an internal (visceral) layer and an external (parietal) layer separated by the pleural cavity. The heart (and other mediastinum contents) are in the central portion of the thoracic cavity and separate the two lungs. Structures like the rib cage help to protect these organs from impacts. The intercostal muscles participate in the breathing process. The air reaches the lungs via the trachea, which branches into the main left and right bronchi. Each of these bronchi subdivide in progressively smaller structures, thus allowing gas exchange with the pulmonary capillaries (Gray et al. (1918)).

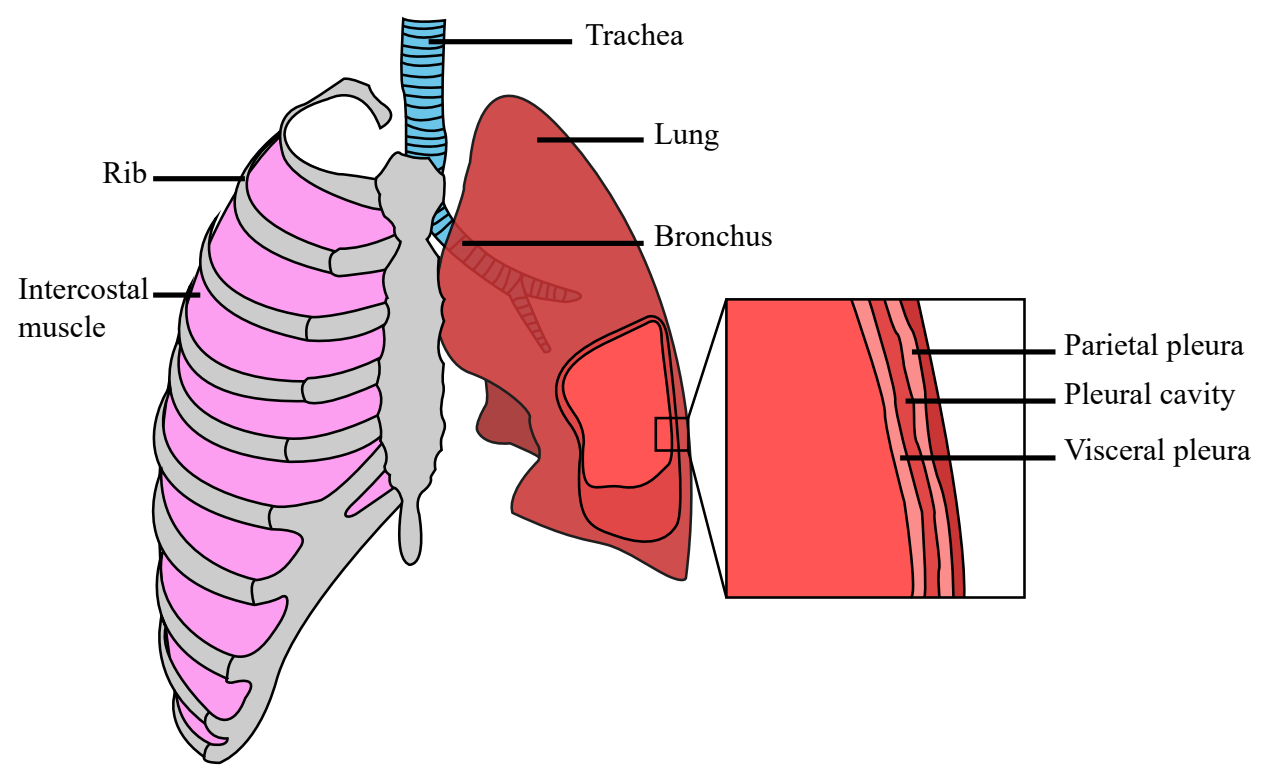

Figure 1.1: Schematic representation of the anatomy of the lungs. Adapted from OpenStaxCollege-Anatomy\&Physiology (2013). 


\subsection{Lung cancer and computed tomography}

Cancer is a group of diseases characterized by an abnormal cell division and spread of these cells to other tissues (NIH (2015)). According to WorldHealthOrganization (2015), "cancer is a leading cause of death worldwide, accounting for 8.2 million deaths in 2012". Among all cancers, lung cancer is the most lethal, being responsible for 1.59 million deaths, i.e, approximately $20 \%$ of cancer related deaths. The 5-year survival rate of patients with lung cancer in developed countries is approximately $20 \%$. This is partially due to the late diagnosis of the pathology. When diagnosed in early stages, the 5-year survival rate increases to over 50\% (De Angelis et al. (2014); Siegel et al. (2015)). Lung cancer is usually a result of unhealthy lifestyles, which include active and passive smoking and exposure to dangerous chemicals (WorldHealthOrganization (2015)).

Lung cancer screening and preliminary diagnosis is performed using non-invasive imaging techniques, including chest X-ray, magnetic resonance and computed tomography (CT). From these, $\mathrm{CT}$ is the most widely used due to the simplicity of the procedure and good imaging results (Swensen et al. (2003); Bach et al. (2012)). A CT image is produced by radiating selected portions of the body with X-photons and measuring the resulting intensity after the traverse. The attenuation value, or tissue density, of a given portion of the body is then assigned to a voxel (Holmes et al. (2008)). It is possible to standardize the attenuation value attributed to each voxel by using Hounsfield units (HU). HU correspond to the comparison of the attenuation value of the tissue with the attenuation value of water. The resulting scale, centered in the HU value of water (zero) (Holmes et al. (2008)), is shown in Fig. 1.2.

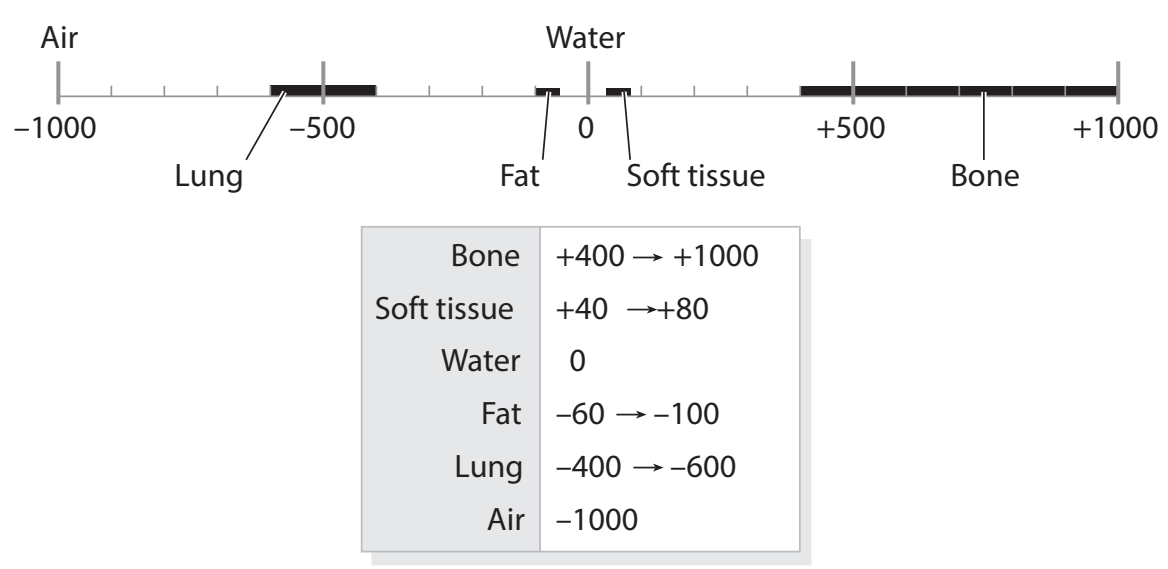

Figure 1.2: Hounsfield unit values for different types of tissue and materials commonly present in a CT scan. -1000 corresponds to black and +1000 to white. Image from Holmes et al. (2008).

In a standardized $\mathrm{CT}$ procedure the equipments are calibrated so that the Hounsfield scale remains true. By maintaining constant the $\mathrm{HU}$, it is possible to easily segment different types of tissues. Note that HU levels may sometimes vary between equipments as result of different radiation energies and other properties. $\mathrm{HU}$ are of interest for diagnosis: by selecting an adequate range of $\mathrm{HU}$ values (i.e., a visualization window), the specialist can analyze different structures and details of interest for the detection of the pathology. 
Multi-slice CT is the most common type of CT imaging. The popularity results from the fact that this technique allows, in relation to its predecessors, to scan large anatomic portions (such as lungs) with low slice thickness. Fig. 1.3 shows an example of a CT scan, as well as relevant anatomical structures. In these slices it is possible to distinguish blood vessels, a fissure (result of the interface between the lung nodules), the pleura and the lung parenchyma.

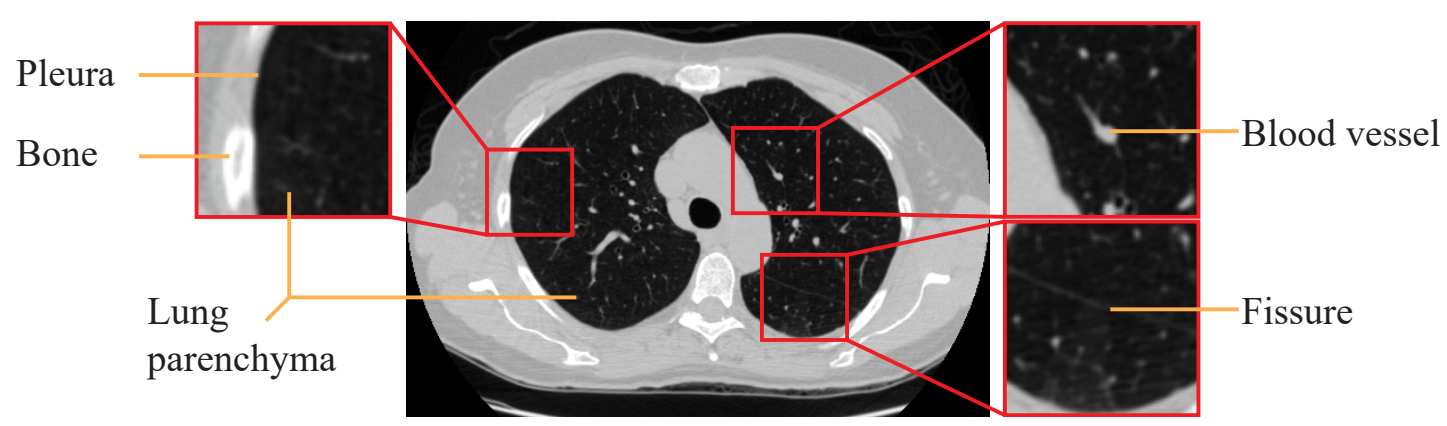

Figure 1.3: Example of a slice from a CT scan. Relevant structures (blood vessels, bone, fissures and lung parenchyma) are depicted. CT scan image from TheCancerImagingArchiveTeam.

Thin slices are important because they allow high-quality 3D reconstructions and reduce the partial-volume effect (Goldman (2008)). The partial-volume effect is the reduction of sharpness of the image and alteration of the image intensity due to the imaging process (Soret et al. (2007)). In a 3D CT image, each voxel intensity is the result of the average attenuation of the respective portion of tissue. When slice thickness increases, the portion of tissue used for assessing the intensity of a given voxe also increases. Due to the averaging, structures become blurred and their overall intensity changes, which is most of the times undesirable for medical imaging analysis.

\subsection{Lung nodule computer-aided detection systems}

The detection of lung cancer cases in CT images is usually performed by trained physicians. In a routine examination, the specialists visually detect the nodules. Nodules are rounded abnormal structures that may indicate primary lung cancers, metastatic diseases or non-cancerous processes (Armato III et al. (2004)). During the procedure, the physicians annotate the nodule positioning and dimension for further follow-up. However, fatigue and distraction errors, together with lack of experience and limitations of the visual system, reduce the detection rate and correct classification of nodules (Valente et al. (2016)). As so, the development of automatic/semi-automatic tools that aid the physicians in nodule detection and diagnosis is of interest. These tools can be generically named as computer-aided detection (CADe) systems and computer-aided diagnosis (CADx) systems. The former has as objective to detect information of interest to the physician (such as the location of possible nodules in a CT scan); the latter is aimed to give a second opinion to the specialist regarding the final diagnosis (Castellino (2005)). This work will contribute to the development of a CADe system for lung cancer screening in CT images. CADe systems can be general or dedicated to a specific nodule type. The combination of dedicated CADe systems 
has shown to improve results in comparison to separated systems (van Ginneken et al. (2010); De Nunzio et al. (2011)). For this purpose, it is important to consider these different types of nodules.

\subsubsection{Types of lung nodules}

Lung nodules can be classified in different ways. The most obvious is perhaps the binary classification as benign or malign. This classification is of interest for CADx systems. Nevertheless, nodules can also be classified according to their texture and location. In CADe systems, the detection process should be adjusted according to the intrinsic characteristics of each kind of nodule.

\subsubsection{Nodule texture}

In terms of texture, nodules can be classified as solid, sub-solid and non-solid (or ground-glass opacities) nodules. Solid nodules (Fig. 1.4a) are those that completely obscure the parenchyma. They are relatively easy to identify, even for less experienced observers, and their margins are usually well defined. Non-solid nodules (Fig. 1.4c) are diffused abnormalities in the parenchyma. The shape of non-solid nodules is highly irregular and difficult to characterize, showing in CT scans as cloudy areas. Sub-solid nodules (Fig. 1.4b) combine the features from solid and nonsolid nodules, only partially obscuring the lung parenchyma.

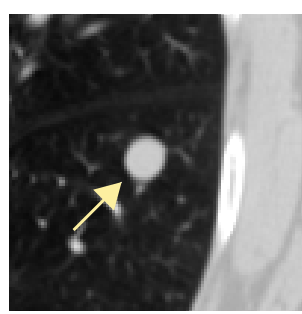

(a) Solid

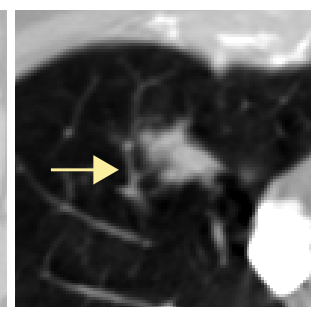

(b) Sub-solid

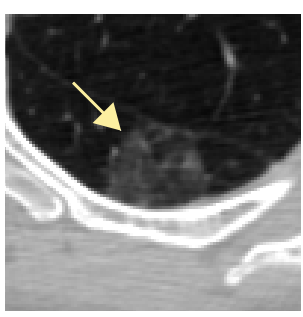

(c) Non-solid

Figure 1.4: Nodule types according to opacity. Images from TheCancerImagingArchiveTeam

\subsubsection{Nodule location}

Nodules can be classified according to their location relatively to other structures in the lungs. According to van Ginneken et al. (2010), lung nodules can be classified as isolated, peri-fissural, juxta-vascular and juxta-pleural. Fig. [1.5 shows an example of each kind of these nodules.

Isolated nodules (Fig. 1.5a) are well-circumscribed rounded structures in the central region of the lung parenchyma. They show no connection to blood vessels, fissures or pleura. These nodules tend to be the simplest to detect using a CADe system, as no special attention to other structures has to be considered. Peri-fissural nodules (Fig. 1.5b) are triangular or oval shapped abnormalities that occur in the periphery of the lung fissures. These nodules are usually non-malignant; nevertheless, their follow-up is still recommended (Ahn et al. (2010)). Juxta-vascular nodules (Fig. 1.5c) are abnormalities that appear attached to vascular structures. In CADe systems, these nodules are challenging to detect as their intensity is similar to the intensity of blood vessels (Sun et al. (2014)). 


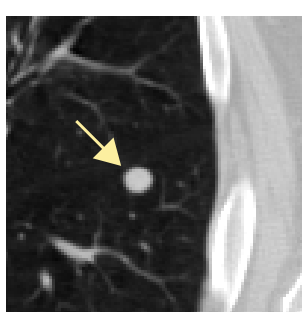

(a) Isolated

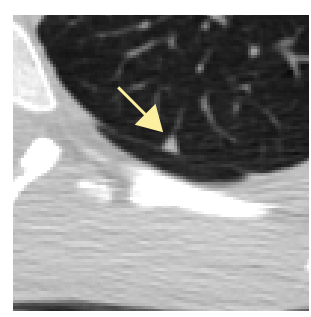

(b) Peri-fissural

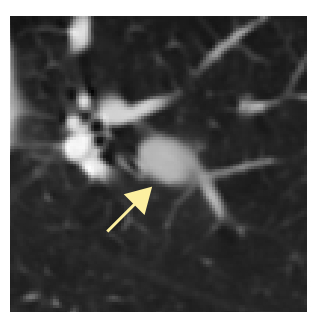

(c) Juxta-vascular

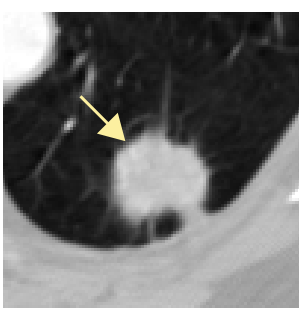

(d) Juxta-pleural

Figure 1.5: Nodule types according to relative location to lung structures. Images from TheCancerImagingArchiveTeam

Juxta-pleural nodules (Fig. 1.5d) are nodules attached to the pleura, the interface membrane between the lung parenchyma and the exterior muscular and fat tissues. Juxta-pleural nodules show different shapes: they can be rounded structures attached by a tail to the pleura (as in Fig. 1.5d or they can be elongated with direct contact to the mentioned membrane. Shape variety and intensity similarity between juxta-pleural nodules and the adjacent tissues difficult the automatic detection of this kind of nodules. In fact, according to van Ginneken et al. (2010), juxta-pleural nodules tend to be the more challenging type of nodule to detect.

\subsubsection{The LIDC/IDRI dataset}

CADe systems must be validated prior to their use in the clinical environment. CADe validation should be performed considering a large range of possible scenarios, as robustness is essential. Large and representative datasets should thus be used for testing CADe performance. Furthermore, the usage of public datasets should be encouraged to ease the comparison between systems.

The Lung Image Database Consortium image collection (Armato et al. (2011); TheCancerImagingArchiveTeam) is one of the largest publicly available dataset aimed at the development of lung nodule detection and classification systems using CT scans. Each scan from the dataset was evaluated by four experienced thoracic radiologists from a universe of twelve specialists. Structures of interest were divided in 3 categories: i) nodule with diameter $<3 \mathrm{~mm}$, ii) nodule with diameter $\geq 3 \mathrm{~mm}$ and iii) non-nodule with diameter $\geq 3 \mathrm{~mm}$. Non-nodules are lung lesions that have no cancerous characteristics (an apical scar, for instance). CADe systems tend to detect these lesions and so their identification is important.

The main focus of the dataset is to promote the study of $\geq 3 \mathrm{~mm}$ diameter nodules. The dataset contains segmentations (ground-truths) of these nodules. Nodules with $\geq 3 \mathrm{~mm}$ diameter also have a subjective evaluation of several characteristics: i) subtlety (difficulty in detection), ii) internal structure/composition (soft tissue, fluid, fat, air), iii) calcification, iv) sphericity (overall roundness of the nodule), v) margin (how well-circumscribed is the nodule), vi) spiculation, vii) texture (solid, non-solid or sub-solid) and viii) malignancy (assuming a 60-year-old male smoker). These characteristics are not usually of interest for CADe systems. However, they can be used for evaluating the performance considering these subjective evaluations. For the smaller nodules 
and non-nodules only the center-of-mass is indicated. Furthermore, regardless nodule size, no location-based classification (isolated, peri-fissural, juxta-vascular or juxta-pleural) is provided.

The ground-truth was established in two phases. In a first phase, each of the four specialists analyzed the scans without access to the work of the others. Segmentations were performed manually or using a semi-automatic software. In the second phase, the ground-truths were revised based on the work of the other radiologists. The radiologists were not forced to segment structures considered as nodules by other radiologists. Consequently, each nodule has assigned an Agreement level. If all radiologists agreed that a given abnormality was a nodule, then the Agreement level is 4. Nodules with higher Agreement level should be easier to detect, as they tend to be more obvious. However, several other factors, namely location, should be taken into account.

The dataset contains 1012 scans, with a total of 7371 nodules. From these, 2669 nodules have a diameter $\geq 3 \mathrm{~mm}$. Approximately $35 \%$ of these nodules have an Agreement level of 4 and 29\% have an Agreement level of 1.

\subsection{Concluding remarks}

Lung cancer is the most lethal type of cancer. The survival rate increases to over $50 \%$ if the diagnosis is performed early. CT scanning is used for lung nodule detection. However, fatigue, distraction and limitations of the human visual system reduce the overall detection and diagnosis performances. CADe systems can help to improve nodule detection rate.

The combination of dedicated CADe systems usually outperforms general approaches. For the different nodule locations (isolated, peri-fissural, juxta-vascular and juxta-pleural), CADe systems tend to perform worse for juxta-pleural nodules. The LIDC/IDRI dataset can be used for the development and performance evaluation of lung nodule CADe systems. Although providing several important characteristics, no nodule location-based classification is given. 


\section{Chapter 2}

\section{Methods for lung nodule detection in CT images}

An automatic lung cancer diagnosis system usually comprises 3 major steps: i) candidate nodule detection, ii) candidate analysis (segmentation and feature extraction) and iii) candidate classification. Candidate detection consists in the determination of the 3D location of the nodules.

The main objective of the proposed work is the development of a lung nodule detection algorithm dedicated to juxta-pleural nodules. The resulting CADe system can either serve as a tool to guide physicians in the location of this type of nodules or integrate a more complex system as the first step indicated above. Ideally, CADe systems should guarantee the correct localization of all the nodules in the scan (true positives, TP) and reduce/eliminate erroneous detections (false positives, FP). A review of works regarding lung nodule detection in CT images is presented in this chapter. Note that in our review it is assumed that the lung volume is already correctly segmented, which may prove to be challenging (Novo et al. (2014)).

The analyzed works were selected by using mainly the following criteria: i) the publishing year should be 2006 or higher (at most 10 years old); ii) validation should preferably be performed in the LIDC/IDRI dataset, simplifying direct comparison between works and iii) the main focus of the work should be nodule detection. The reviewed works were researched using GoogleScholar, Scopus and ScienceDirect search engines. The keywords used for searching general lung nodule detection approaches were lung, nodule detection, CT. For juxta-pleural nodules, the used keywords were pleural, juxtapleura, juxtapleural, juxta-pleura, juxta-pleural and nodule detection.

\subsection{Lung nodule detection steps}

The overall structure of a lung nodule detection algorithm is depicted in Fig. 2.1.

A lung nodule detection algorithm is usually comprised by 3 parts: i) candidate detection (section 2.1.1), ii) candidate refinement (section 2.1.2) and iii) FP reduction (section 2.1.3). An explanation of each of these parts is given in the remaining of this section. Special attention should 


\begin{tabular}{|c|c|c|}
\hline Candidate detection & Candidate refinement & False positive reduction \\
\hline $\begin{array}{l}\text { Hounsfield unit } \\
\text { based threshold }\end{array}$ & $\begin{array}{l}\text { Removal of attached } \\
\text { structures }\end{array}$ & $\begin{array}{l}\text { Rule-based } \\
\text { (volume and diameter) }\end{array}$ \\
\hline Blob detector & $\begin{array}{l}\text { Nodule region } \\
\text { growing }\end{array}$ & Classifier-based \\
\hline Shape analysis & & $\begin{array}{l}\text { networks, support } \\
\text { vector machines, ...) }\end{array}$ \\
\hline
\end{tabular}

Figure 2.1: Overall structure of a lung nodule detection algorithm.

be given to shape analysis and active contour methods, for candidate detection and refinement, respectively, as they can be used for juxta-pleural nodule detection.

\subsubsection{Candidate detection}

The first step is the detection of nodules candidates. This procedure should ideally guarantee a $100 \%$ sensitivity, i.e., all the existing nodules should be detected. Usually, the resulting number of FP is very high as we are still in an initial detection stage. The initial candidate detection can be achieved by HU intensity-based thresholding, by using blob detectors or by using region growing of the parenchyma to detect the regions/structures of interest. The latter is of interest for the detection of juxta-pleural nodules, because juxta-pleural nodules with solid texture cause deformations in the limits of the lung mask.

HU intensity-based methods The standardization of CT imaging techniques allows the fast identification of anatomic structures via HU intensity-based threshold (Fig. 1.2). The simplicity and low computational costs make this method attractive for initial candidate location ( $\mathrm{Pu}$ et al. (2008); Messay et al. (2010); Han et al. (2015); Jacobs et al. (2014); Setio et al. (2015)). These methods allow to obtain both location and a rough segmentation of the candidates. HU thresholding can be performed considering one or multiple threshold levels. For instance, Setio et al. (2015) used an intensity threshold of $-300 \mathrm{HU}$ to identify solid nodules. Alilou et al. (2014), on the other hand, combined the result of multiple threshold levels to identify lung nodules. Note that, by themselves, HU intensity-based threshold methods seem to not be viable for juxta-pleural nodule location due to the intensity similarity between nodules and fat tissue (Taşc1 and Uğur (2015)). They can, however, be of interest for preliminary detections if a proper lung segmentation is available.

Region growing of the parenchyma, based on known HU values, can also be used for the initial detection of candidates. Seed points in the parenchyma are selected via analysis of the HU histogram. The intensity of the neighbor pixels of the seed points is studied. If the pixels respect a given threshold criteria, they are included in the region. The threshold is reestablished based on the new region. The process is performed iteratively until no more pixels are added (Ridler and 
Calvard (1978)). Once the region growing process is complete, the candidates can be obtained by subtracting the resulting mask to a lung mask (Cascio et al. (2012)).

Blob detectors Blob detection-based methods are used for enhancement or detection of rounded/ spherical structures. The rounded shape of the nodules makes blob detectors a viable method for initial candidate location. Furthermore, these methods, unlike simple thresholding, are more robust to the presence of adjacent structures, such as vessels. A common blob detector has the following stages: for all voxels, a $3 \times 3$ Hessian matrix is calculated by using the response of the second order derivative of a 3D-equivalent Gaussian curve. Then, the eigenvalues $\left|\lambda_{3}\right| \leq\left|\lambda_{2}\right| \leq\left|\lambda_{1}\right|$ are retrieved. The eigenvalues allow to compute blobness metrics. Retico et al. (2008); Guo and Li (2012); Choi and Choi (2014) used the metric described in Li et al. (2003):

$$
z_{\text {dot }}\left(\lambda_{1}, \lambda_{2}, \lambda_{3}\right)= \begin{cases}\left|\lambda_{3}\right|^{2} /\left|\lambda_{1}\right| & \text { if } \lambda_{1}<0, \lambda_{2}<0, \lambda_{3}<0 \\ 0 & \text { if otherwise }\end{cases}
$$

Murphy et al. (2009); Novo et al. (2015) consider the shape index (SI) and curvedness (CV):

$$
S I=\frac{2}{\pi} \arctan \left(\frac{\lambda_{1}+\lambda_{2}}{\lambda_{1}-\lambda_{2}}\right), C V=\sqrt{\lambda_{1}^{2}+\lambda_{2}^{2}}
$$

The obtained values are used for the detection of the candidates. For this purpose, a fixed threshold, average of local maximum or other thresholding techniques can be used. Blob detectors can consider one or multiple scales. For the latter, the maximum response for each scale is combined for the final output. Multiple scale approaches usually show better results as the filter responses for several nodule diameters can be analyzed.

Shape analysis methods Juxta-pleural nodules are attached to the boundaries of the lungs. Also, these nodules have a similar intensity to non-parenchymal tissue. As a consequence, lung segmentation masks usually include deformations/concavities caused by the presence of these nodules ( $\overline{\mathrm{De}}$ Nunzio et al. (2011)). Detection of juxta-pleural lung nodules can be performed by detecting concavities in the lung mask. Concavities can be found by using $\alpha$-hulls (De Nunzio et al. (2011); Taşc1 and Uğur (2015)). The $\alpha$-hull is a generalization of the convex-hull concept. The shape of the $\alpha$-hull depends on the parameter $\alpha$ : given a set of points $\mathrm{S}$, "the $\alpha$-hull of $\mathrm{S}$ is the intersection of the closed components of all the circles of radius $r=1 / \alpha$, such that the intersection of these circles with $\mathrm{S}$ is empty" (De Nunzio et al. (2011)). A low $\alpha$ value means higher curvature radius of the hull; in the limit, if $\alpha=0$, a convex-hull is obtained. By using different values of $\alpha$ and by subtracting the resulting hulls to the initial mask, it is possible to find the concavities. This method also allows to obtain a segmentation of the candidate. Fig. 2.2 illustrates the discussed process.

Juxta-pleura nodules can be detected by evaluating the directional-gradient concentration, as described in Retico et al. (2009). The directional-gradient concentration consists in the analysis of the number of intersections of the surfaces normals. The pleural surface can be discretized by a finite number of triangular elements. Each of these triangles has a vector normal to its surface. 

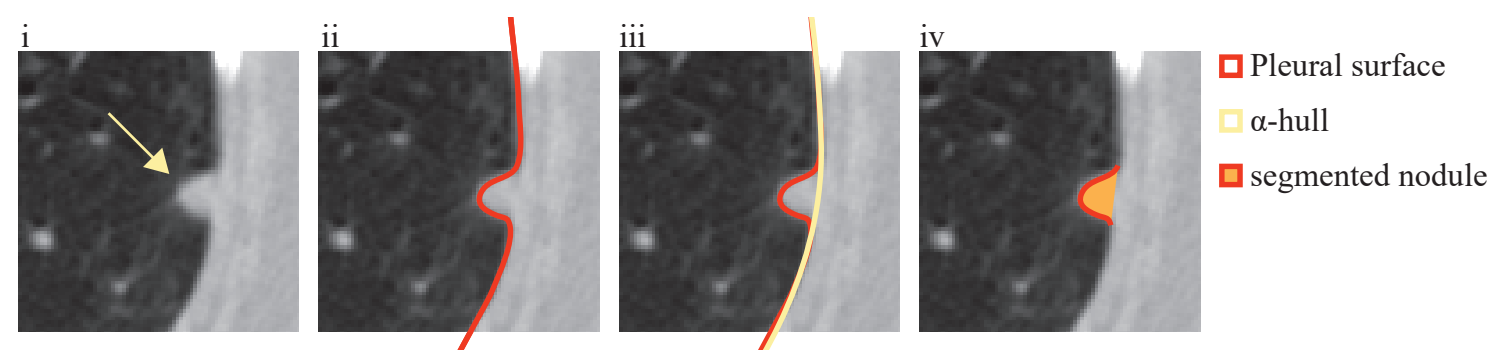

Figure 2.2: Steps for juxta-pleural lung nodule detection using $\alpha$-hulls. i) juxta-pleural lung nodule; ii) pleural surface; iii) $\alpha$-hull partially overlaying the pleural surface; iv) segmented nodule, resulting from the subtraction of the $\alpha$-hull to the pleural surface.

The voxels adjacent to the pleural surface are scored according to the number of intersections of normal directional lines that occur in it. Regions with high concentration of intersections indicate prominences in the pleura, which, by its turn, are indicative of the presence of lung nodules. The detection method using the directional-gradient concentration is illustrated in Fig. 2.3.
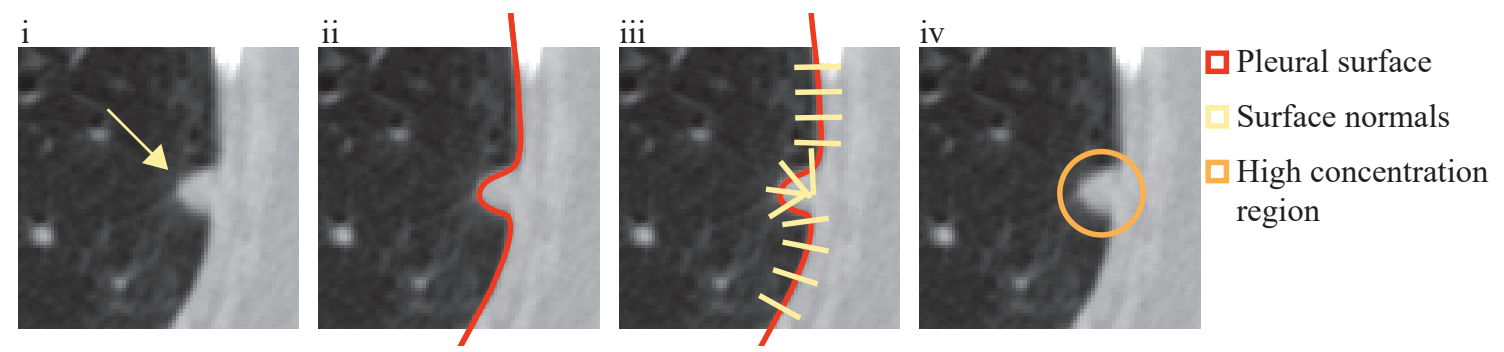

Figure 2.3: Steps for juxta-pleural lung nodule detection using directional-gradient concentration. i) juxta-pleural lung nodule; ii) pleural surface; iii) normals to the surface; iv) detection of the nodule as the region with higher surface normals overlap.

\subsubsection{Candidate refinement}

The candidate detection step usually results either in a rough segmentation or in the approximate coordinates of the centroid of the nodule candidates. Also, the number of FP candidates is high. Prior to reducing the number of FP, it is necessary to remove possible attached structures (such as vessels or the pleural wall) and correct the segmentation of the nodule. The removal of connected structures is usually performed using morphological operations. Segmentation can either be performed by intensity-based region growing or by using active contours techniques.

Removal of attached structures Structures like vessels or the pleural wall have HU values similar to lung nodules. Lung nodules can appear near vessels and the pleural wall (juxta-vascular and juxta-pleural nodules, respectively). As a consequence, after candidate detection, these nodules may be attached to these structures. The removal of the attached structures can be performed using morphological operations (Okada et al. (2005); Messay et al. (2010); Badura and Pietka (2014)). A binary structuring element is shifted over the binary mask of the candidates. If the 
structuring element matches partially or completely the current portion of the mask, the respective pixel of interest is changed to 1 (dilation) or 0 (erosion). Opening, which is an erosion followed by a dilation using the same structuring element, is often used. Both 2D and 3D morphological operations can be used. 2D operations are less computationally complex, which may prove to be advantageous (Messay et al. (2010)).

Intensity-based region growing Nodule segmentation can be performed using intensity-based region growing. The seed points needed for region growing are obtained from the rough segmentation resulting from the initial candidate detection or by considering the candidates' centroid. 3D region growing is a common candidate refinement technique (Murphy et al. (2009); Guo and Li (2012); Brown et al. (2014); Setio et al. (2015)). As stated in section 2.1.1, a region growing algorithm is an iterative process of inclusion of neighbor pixels with similar characteristics. In a 3D algorithm, the neighbors from the same slice and adjacent slices are analyzed. Region growing algorithms are effective for the segmentation of isolated nodules. However, using region growing in nodules near other anatomic structures may result in the inclusion of these structures in the candidate due to the similarity in intensity.

Active contour Active contours are a computer vision technique that allow to segment images by delineation of the structure of interest. Due to their complexity but good results, active contours are often used for nodule classification instead of nodule detection, as shape is an essential characteristic to classify a nodule (Armato et al. (2011)). Nevertheless, active contour techniques can also be used for candidate refinement in nodule detection algorithms (Cascio et al. (2012)). This technique may be of interest for juxta-pleural nodule detection as it is more robust to the inclusion of adjacent structures than region growing. A standard shape is initialized in the candidate region. Then, the position of interrelated points is iteratively updated considering two types of energy, internal and external. The internal energy $\left(\right.$ Energy $\left._{\text {internal }}\right)$ is inherent to the model itself. It regulates how the model contracts and curves to adapt to the shape to segment. External energy, on the other hand, regards the contribution of the image to the final shape. External energy (External energy $)$ is a function of the gradient of the image intensity. For segmentation of lung nodules, higher gradient values and low intensity values imply lower external energy. The objective of the process is to minimize the overall model energy, resulting from the contribution of each model point $p$ (Terzopoulos and Fleischer (1988); Cascio et al. (2012)):

$$
\operatorname{Energy}_{\text {total }}=\min \left(\sum_{p}\left[\operatorname{Energy}_{\text {internal }}(p)+\operatorname{Energy~}_{\text {external }}(p)\right]\right)
$$

\subsubsection{False positive reduction}

The initial candidate detection produces a high number of FPs. The candidate refinement step allows to improve the segmentation of the candidates. The last step is the reduction of the number of FPs based on the characteristics of the candidates. Two procedures are used: first, a ruled-based 
analysis is performed to eliminate candidates that obviously are not nodules; then, features are retrieved from the remaining candidates for classification in nodule or non-nodule.

Rule-based false positive reduction The majority of works addressing lung nodule detection focus on nodules with diameter ranging approximately between $\left[\begin{array}{ll}3 & 30\end{array}\right](\mathrm{mm})$. This happens because nodules with higher radius are easy to detect and small nodules appear to not have much importance for immediate diagnosis (Setio et al. (2015); Valente et al. (2016)). Both diameter and volume (equivalent to a sphere of the same diameter) are commonly used for eliminating candidates that obviously are not lung nodules (Cascio et al. (2012); Alilou et al. (2014); Han et al. (2015); Setio et al. (2015)). It is important that no TP are removed during this process. Consequently, a more relaxed criteria should be used if the candidates tend to attach to other structures.

Classifier-based false positive reduction Rule-based restrictions allow to reduce the number of FPs. However, the number is still too high. A more refined candidate selection is performed using classifiers. Classification consists in the use of a set of features to give a category to a new observation (Smola and Vishwanathan (2014)). Prior to the classification, the classifier must be trained using features from known observations (such as the ground-truth). Classifier training can be supervised, if training labels (categories) are provided or unsupervised if that does not happen. Supervised learning is usually used for FP reduction by considering the labels nodule or nonnodule. The most common features are geometrical (volume, diameter, number of voxels), shaperelated (using the Hessian matrix to assess blobness, for instance) and intensity-based (Murphy et al. (2009); Tan et al. (2013); Taşc1 and Uğur (2015)). Furthermore, Choi and Choi (2014) used the histogram of the surface normals and Setio et al. (2015) considered the location of the nodules as a feature for classification.

Several types of classifiers are used for lung nodule candidate FP reduction. Some of the most used classifiers are support-vector-machines (SVM) (de Carvalho Filho et al. (2014); Valente et al. (2016)), feed-forward neural (FF) networks (Retico et al. (2008); van Ginneken et al. (2010); Lopez Torres et al. (2015)) and the k-nearest neighbor algorithm (kNN) van Ginneken et al. (2010); Valente et al. (2016)). According to Platt (1998), SVM is, in the linear form, a hyperplane that separates two sets of data while maximizing the minimum distance of the two sets from that plane (i.e., maximizing the margin). Generally, this hyperplane is the result of the mapping of the input space to a feature space using a nonlinear map (Hearst et al. (1998)). Fig. 2.4a depicts the principle of the SVM.

Feed forward neural networks are a kind of artificial neural networks where information travels in a single direction, from input to output. According to Davidian (1995), FF networks are usually composed by an input layer, where features are fed, hidden layers, where information is processed, and an output layer that gives the final classification. Each hidden layer is composed by a certain number of neurons placed in parallel. By its turn, each neuron weights a given combination of the inputs of the previous layer processed by an activation function (a sigmoid function, for instance). 


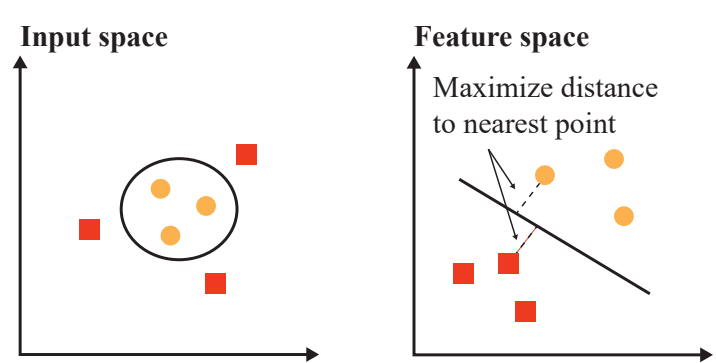

(a) Support vector machines can be considered as a hyperplane that maximizes the minimum distance from that plane. Image adapted from Platt (1998); Hearst et al. (1998).

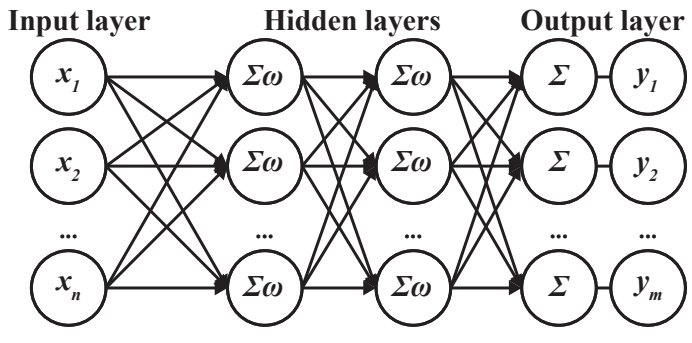

(b) Standard architecture of a feed-forward neural network. $x$ is the input, $y$ the output and $\omega$ is an activation function. Image adapted from WolframMathematica

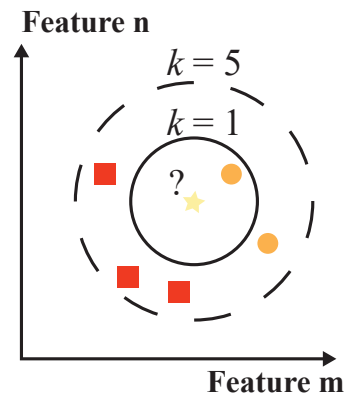

(c) k-nearest neighbors algorithm considering different $k$ values. The unknown observation $(\star)$ is labeled as $\bullet$ if $k=1$ and as $\boldsymbol{\square}$ if $k=5$. Imaged adapted from Ajanki (2007).

Figure 2.4: Commonly used classifiers for false positive reduction of nodule candidates.

The training of the FF network is performed iteratively by feeding new data until the best result is achieved. A standard architecture of a FF network is shown in Fig. 2.4b.

The kNN algorithm (Wu et al. (2008)) classifies a new observation feature vector by evaluating the distance of each feature to the $k$ nearest labeled observations (neighbors). The label of the new observation corresponds to the most common label among the neighbors. The Euclidean distance can be used for finding the nearest neighbors. The classification depends on the parameter $k$, as represented in Fig. $2.4 \mathrm{c}$.

\subsection{Representative lung nodule detection algorithms}

Some of the most representative works for lung nodule detection in CT images, both in terms of performance and detection technique, are now detailed. Please refer to section 2.1 for a comprehensive description of the methods used. The performance of the methods is shown using sensitivity per a given average number of FP/scan. The discussed works used the LIDC-IDRI dataset.

Cascio et al. (2012) used 3D mass-spring models for nodule detection. First, seed points are selected by subtracting a region growing of the parenchyma to a mask of the lung volume. A spherical mesh is initialized for each of the seeds. The position of each of the sphere's vertexes 
is updated in order to minimize the model's energy (similar to an active contour model). Spline curves are then used for reconstructing the surface of the candidate. FPs are reduced by removing all candidates with diameter $\notin\left[\begin{array}{ll}350 & 5\end{array}\right.$ ( $\left.\mathrm{mm}\right)$. Geometrical and intensity distribution features are then retrieved. FP reduction is further performed using a trained three layer feed forward neural network. The algorithm achieved $88 \%$ sensitivity $(2.5 \mathrm{FP} / \mathrm{scan})$ and $97 \%(6.1 \mathrm{FP} / \mathrm{scan})$.

Alilou et al. (2014) used a multiple threshold method to detect nodules candidates. Fixed HU threshold levels were determined by examination of the dataset. For each threshold level, residual structures such as attached vessels are eliminated with a 2D morphological opening. Then, blobs with diameter $\notin[430](\mathrm{mm})$ are removed. The resulting binary masks of all levels are combined using logical "OR". A greedy forward method was used for selecting 3D geometrical, 3D intensity-based, 2D geometrical and 2D intensity-based features. A SVM classifier with a radialbased-function kernel labels the candidates as "nodule" or "non-nodule". The method achieved a $80 \%$ sensitivity with $3.9 \mathrm{FP} / \mathrm{scan}$ and a maximum sensitivity of $90 \%$.

Choi and Choi (2014) developed a detection method based on a angular histogram of surface normals (AHSN) feature. First, candidate nodules are detected by using a multi-scale Gaussian blob detector technique. For each scale, the threshold considers the average of the local maximum dot values (value based on the eigenvalues of the Hessian matrix). Using eigenvalues of the Hessian matrix, the histogram of the surfaceness of the candidate is obtained. Histogram peaks corresponding to walls are removed. This histogram is used in a trained SVM classifier to reduce the number of FPs. This method obtained a sensitivity of $97.5 \%$ with $6.76 \mathrm{PF} / \mathrm{scan}$.

Han et al. (2015) proposed a vector quantization-based approach to detect nodules in CT scans. The histogram of the lungs is represented by 4 Gaussian curves, which correspond to low-frequency parenchyma, high-frequency parenchyma, blood vessels and nodule candidates. Nodule candidates are considered to belong to the class with the highest average intensity. Vessels and other structures are removed using morphological opening. The number of FP was reduced by experimentally determining the volume-equivalent diameter, 3D elongation and compactness. A SVM classifier with a radial-basis-function considering intensity, geometric and Hessian features further reduces the number of FPs. The algorithm showed a sensitivity of $82.7 \%$ (4.0 FP/scan) for all types of nodules and 89.2\% (4.14 FP/scan) for juxta-pleural nodules.

Setio et al. (2015) studied large solid nodule $(>10 \mathrm{~mm}$ ) detection. A fixed HU threshold is used. Then, a multiple stage morphological opening removes attached structures. Nodule candidates with diameter $\notin[840](\mathrm{mm})$ are discarded. Region growing is used for segmenting the remaining candidates. The same size threshold is re-applied. A SVM classifier is used for reducing the number of FPs by considering features as intensity, geometry, blobness (Hessian-based) and location (distance to lung structures). The method has a sensitivity of $98.3 \%$ (4.0 FP/scan).

\subsubsection{Juxta-pleural nodules specific approaches}

The detection of juxta-pleural nodules can be more challenging than other types of nodules. Juxtapleural nodules are present near the boundaries of the lung. As so, a poor lung segmentation can eliminate these structures. Assuming that the lung segmentation is successful, other problems still 
remain. Segmentation techniques, such as region-growing, are affected by the intensity of the surrounding tissue and the presence of other structures such as bone. Portions of the pleura are usually included during the segmentation process, affecting the performance of the system.

Most works study general approaches, attempting to detect several types of nodules using the same approach (Valente et al. (2016)). To the best of our knowledge there are few works addressing specifically juxta-pleural nodule detection. Three relevant works are described.

Retico et al. (2009) used a directional-gradient concentration analysis to determine the location of juxta-pleural nodules. The pleural surface is retrieved from the segmentation of the lungs using a HU-based threshold. A discrete 3D model, composed of triangular elements, is created by using a marching cube algorithm. The authors evaluate the pleural surface normals. Regions with high surface normals intersections, such as prominent nodules, are considered as candidates. The candidates are detached from the pleural wall by using morphological opening. Geometrical and textural features are used in a feed-forward neural network to reduce the number of FP. The method showed to be susceptible to irregularities of the pleural surface, achieving a sensitivity of $72 \%$ with $6 \mathrm{FP} / \mathrm{scan}$. The evaluation was performed in a private dataset.

De Nunzio et al. (2011) proposed an $\alpha$-hull based system. The lungs were segmented using a region-growing based approach followed by a refinement with morphological operations. The $\alpha$-hull is a generalization of the convex-hull method. The authors used $\alpha$-hull to search for concavities in the lung mask, which result from dense juxta-pleural nodules. The approach uses $\alpha$ values equivalent to the nodule radii to detect the candidates. After, geometrical and intensitybased features are extracted. The number of FP is reduced by using a feed-forward neural network. The maximum sensitivity was $92.3 \%$. Taşc1 and Uğur (2015) proposed a similar approach. FP reduction is done using geometrical and texture features with a generalized linear regression model. The obtained sensitivity is not stated.

\subsection{Results review}

A summary of the sensitivity vs $F P /$ scan results for different nodule detection algorithms (not exclusively dedicated to juxta-pleural) is shown in Fig. 2.5. All the analyzed methods were evaluated in the LIDC/IDRI dataset (Armato et al. (2011)). The number of scans used for obtaining the results differs between authors, ranging between 58 and 888. Almost all works studied nodules with a minimum diameter of $3 \mathrm{~mm}$. Slice thickness varies between 0.5 and $3 \mathrm{~mm}$. The average sensitivity of the analyzed state-of-the art methods is $82 \%$ at an average FP rate of 4.1 per scan.

The evaluation of the methods' performance should consider both sensitivity and FP/scan. Higher sensitivity indicates an increase in the overall capacity of detecting TPs. The performance increases with the sensitivity and decreases with the number of FP/scan. Empirically, the approaches proposed by Cascio et al. (2012), Choi and Choi (2013) and Setio et al. (2015) show the highest performance. However, this conclusion would only be truly valid if all the methods were tested in the same test set with the same conditions. Slice thickness, for instance, is known to affect the performance of algorithms due to the partial-volume effect. High thickness, or poor 


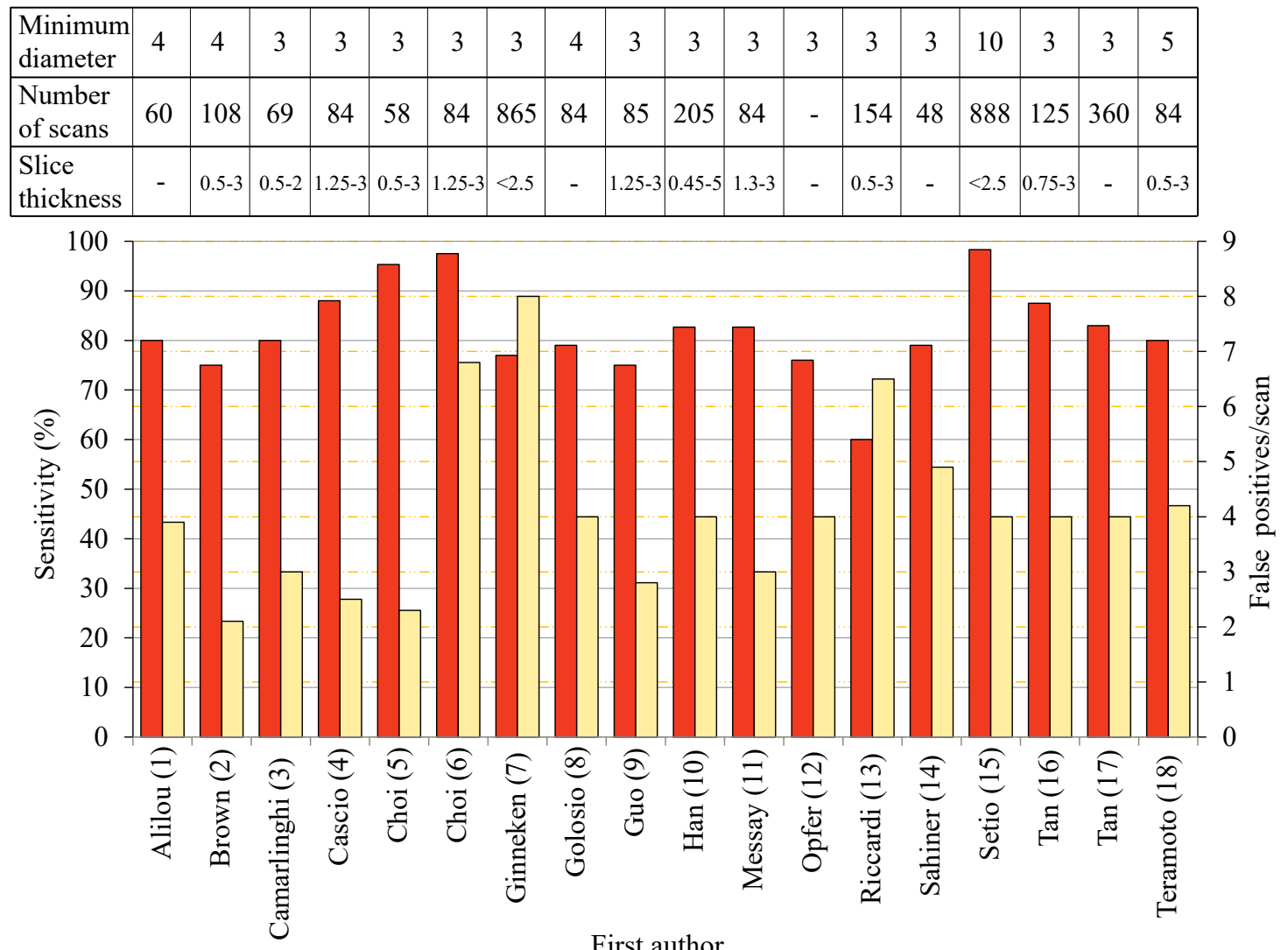

$\square$ Sensitivity (\%) $\quad$ False-positives/scan

Figure 2.5: Sensitivity (\%) and respective number of false positives/scan for different methods. The minimum nodule diameter $(\mathrm{mm})$, range of slice thickness $(\mathrm{mm})$ and number of scans studied are also shown. Values retrieved from: 1) Alilou et al. (2014); 2) Brown et al. (2014); 3) Camarlinghi et al. (2012); 4) Cascio et al. (2012); 5) Choi and Choi (2013); 6) Choi and Choi (2014); 7) van Ginneken et al. (2015); 8) Golosio et al. (2009); 9) Guo and Li (2012); 10) Han et al. (2015); 11) Messay et al. (2010); 12) Opfer and Wiemker (2007); 13) Setio et al. (2015); 14) Han et al. (2015); 15) Setio et al. (2015); 16) Tan et al. (2011); Setio et al. (2015); 17) Tan et al. (2013); 18) Teramoto and Fujita (2013).

resolution in the direction normal to the anatomical axial plane, leads to discretization and nodule blurring, hindering results (van Ginneken et al. (2010); Brown et al. (2014)). The hit criterion should also be taken into consideration, as it defines if a final candidate should be classified as TP or FP. A common method is to evaluate the distance between the candidate and the ground truth. For instance, if the center of candidate lies in a sphere centered in the ground truth and with $1.5 \times$ the radius of the ground truth, then the candidate is a TP. Once hit, the respective ground truth should be removed from the analysis list to avoid false TPs. The hit criterion is commonly not stated by the authors, which affects the validation and evaluation of the results. Setio et al. (2015) have a high sensitivity value with a low FP/scan $(98.4 \%$; $4 \mathrm{FP} / \mathrm{scan})$. However, they only studied large nodules, which are more easy to detect, segment and classify. Furthermore, they did not consider as FP all the candidates that hit non-nodule structures. The method proposed by Choi 
and Choi (2013) seems to be the one with the highest performance (95.3\%;2.3 FP/scan). However, the number of scans studied is low when compared with other works. The small number of cases does not show if algorithm would be capable of maintaining the same performance with a second set of images. Also, the hit criterion presented by the authors is dubious as it does not clearly state if the ground truth of a given nodule is only considered once.

Based on the studied steps of the methods it is difficult to state which combination provides a better overall performance. Several detection methods are used but the most common tend to be HU-based threshold and blob detectors. In terms of candidate refinement, the revision of the works indicates that active contour-based methods show a good performance, followed by region growing. FP using rules seems to greatly enhance results and is commonly used. Both SVM and FF networks greatly reduce the number of FP. To conclude, a high sensitivity initial candidate detection with a low number of FP followed by a good FP reduction classifier step should be the goal of a lung nodule CADe detection system.

\subsubsection{Detection per nodule type}

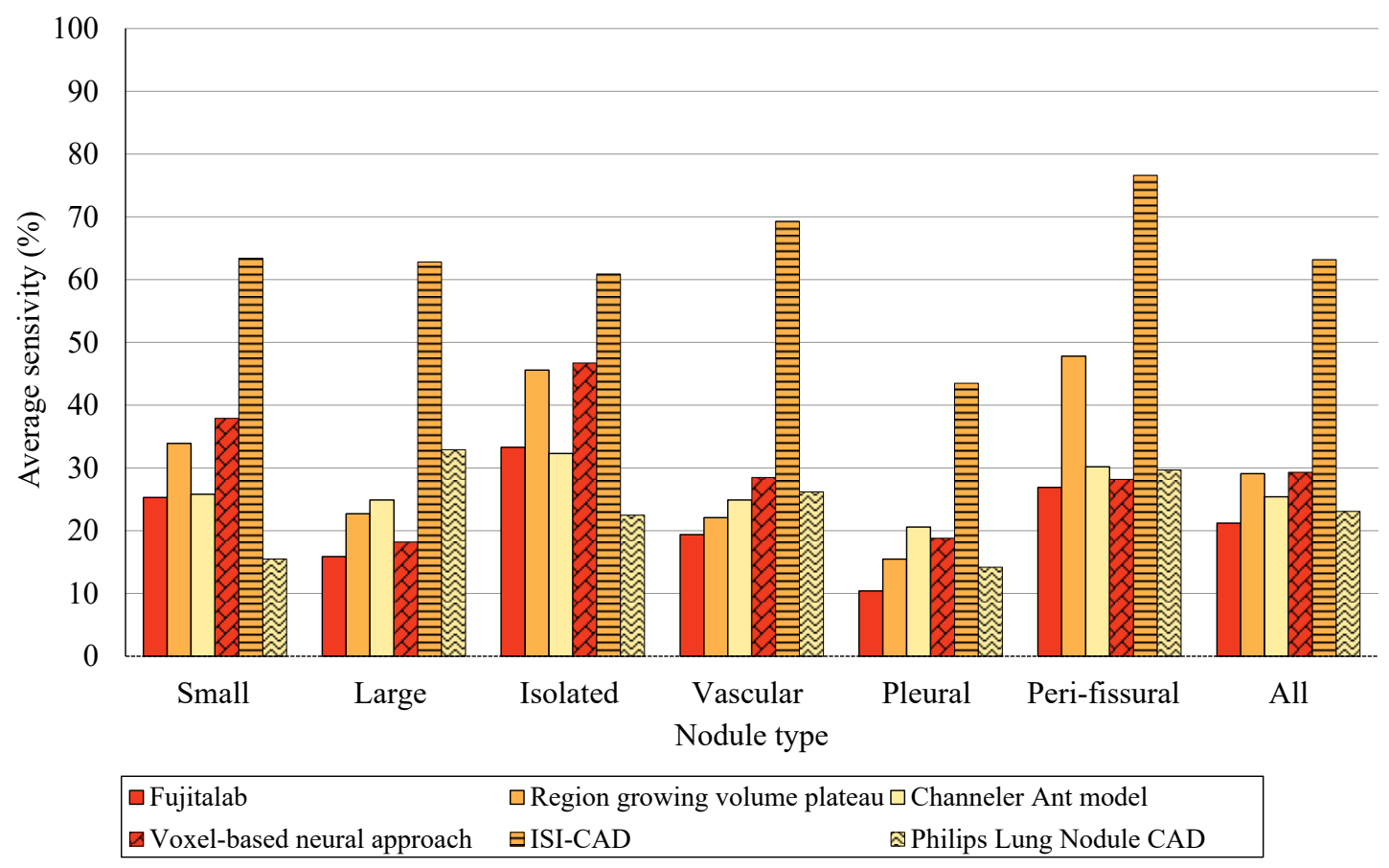

Figure 2.6: Average sensitivity values of several rates of false positives/scan $\left(2^{-3}, 2^{-2}, \ldots, 2^{3}\right)$ for different type of nodules and algorithms. Data retrieved from van Ginneken et al. (2010).

Fig. 2.6, based on van Ginneken et al. (2010), shows the performance in terms of average sensitivity of different detection algorithms for various nodule types: i) small (diameter $<5 \mathrm{~mm}$ ), ii) large (diameter $\geq 5 \mathrm{~mm}$ ), iii) isolated, iv) juxta-vascular, v) juxta-pleural and vi) peri-fissural. The sensitivity was evaluated for different numbers of false positives/scan $\left(2^{-3}, 2^{-2}, \ldots, 2^{3}\right)$ for each of the studied algorithms. The average sensitivity (per algorithm) was used for comparison 
(Fig. 2.6. The performance of the algorithms was analyzed using the dataset from the ANODE09 (2009) challenge. This dataset contains mainly solid nodules, which are characterized per location. As the evaluation was not performed using the LIDC/IDRI dataset, direct comparison with the lung nodule candidate detection methods revised previously does not allow to draw defining conclusions. However, the average of the results of the different methods ( $\eta_{\text {noduletype }}$ ) allows to assess the difficulty in detecting each kind of nodule.

The six nodule detection algorithms are: i) Fujitalab, ii) Region growing volume plateau, iii) Channeler Ant model, iv) Voxel-based neural approach, v) ISI-CAD and vi) Philips Lung Nodule CAD (commercial product). Fujitalab detecs nodules using a gradient concentration filter to enhance rounded structures followed by region growing to detect and segment candidates. FPs are first reduced with rules and after with a multi-regression analysis. Region growing volume plateau uses region growing of the parenchyma to detect seed points, followed by a second region growing for nodule segmentation. After rule-based FP reduction, a FF network is used. The Channeler Ant model uses a dynamic region growing process (homonymous to the work) to detect candidates. Classification is performed using a FF network. The Voxel-based neural approach uses blob detectors to detect nodules in the parenchyma and the directional-gradient concentration to detect juxta-pleural nodules. Features are extracted from the surrounding region and classified using a FF network. ISI-CAD computes the shape index and curvedness to detect candidates. Then, two k-kNN classifiers are used, sequentially with different features, to reduce the number of FPs. Please refer to van Ginneken et al. (2010) for a description of each of the methods studied.

Fig. 2.6 allows to draw several conclusions. First, isolated nodules $\left(\eta_{\text {isolated }}=40.2 \pm 13.6\right)$ show a tendency of higher sensitivity among all type of nodules, i.e., they appear to be the easiest to detect. The good intensity contrast between these nodules and the parenchyma contributes to the obtained sensitivity. Furthermore, the segmentation step is not affected by surrounding structures, such as vessels or the pleural. In fact, nodules that are not attached to large structures, such as isolated and peri-fissural ( $\eta_{\text {perifissural }}=39.9 \pm 19.6$ ) show higher $\eta$ values than the juxta-vascular $\left(\eta_{\text {juxtavascular }}=31.7 \pm 18.7\right)$ and the juxta-pleural $\left(\eta_{\text {juxtapleural }}=20.5 \pm 11.8\right)$ nodules.

Juxta-pleural nodules show tendency to have the lowest average sensitivity from the analyzed types of nodules. This is true even for the Voxel-based neural approach, which used a dedicated approach for this type of nodule. Overall, this can be justified by the lack of contrast between the nodule and the surrounding tissue and the existence of adjacent structures, which hinder both initial detection and segmentation. Considering the works showed in Fig. 2.6, blob detectors seem to outperform other methods in terms of juxta-pleural nodule detection. However, the performance of the blob detector method for juxta-pleural nodule detection is still lower when compared to other nodules types. Based on the discussed $\eta$ values, there is need to develop algorithms specially dedicated to the detection of juxta-pleural nodules. 


\subsection{Concluding remarks}

Nodule detection algorithms are often composed by an initial candidate detection step, followed by a candidate refinement and reduction of FPs. Initial candidate detection can be performed using methods based on the HU values, by analyzing shape-related characteristics or by using blob detectors. For candidate refinement, morphological operations are used for detaching nonnodule structures from the candidates. Then, region growing or active contours can be used for obtaining a better segmentation of the candidates. The number of FPs is reduced by using fixed rules and classifiers. Several works focused on lung nodule detection in CT images were studied. The detection algorithms show similarities between them, with the main differences occurring in the method used to find the initial candidates.

The detection of juxta-pleural nodules shows to be challenging due to their position near the pleural wall. However, to the best of our knowledge there are few works addressing specifically the detection of this kind of nodules. Initial candidate detection can be performed by using $\alpha$ shapes to detect concavities, by analyzing the directional-gradient concentration or by using blob detectors. Direct thresholding techniques using HU values are avoidable due to the presence of the pleural wall, except when a proper lung volume segmentation is available. Candidate refinement should be performed by methods other than region-growing, as there is risk to attach a portion of the pleura and adjacent structures to the candidate. Morphological operations are important to detach candidates. Finally, FP reduction should have in consideration not only intensity-related features, but also the nodule geometry and location. SVM, FF networks and the k-NN algorithm are often used for FP reduction.

Based on the unique characteristics of the juxta-pleural nodules and the performance of the reviewed methods, the algorithms proposed by Retico et al. (2009); De Nunzio et al. (2011); Cascio et al. (2012); Choi and Choi (2013); Han et al. (2015); Taşc1 and Uğur (2015), as well as the features proposed in Setio et al. (2015), are of major interest to the development of a novel CADe algorithm dedicated to the detection of juxta-pleural nodules. 


\section{Chapter 3}

\section{Juxta-pleural lung nodule detection}

\subsection{Method overview}

The proposed method to detect juxta-pleural nodules in CT scans is detailed in this chapter. Previous works have achieved high detection performance for nodules with radius $\geq 5 \mathrm{~mm}$, including juxta-pleural (Setio et al. (2015)). Consequently, our work focuses on the detection of juxta-pleural nodules with radius $\leq 5 \mathrm{~mm}$. Our approach can be divided in:

1. lung volume segmentation with inclusion of juxta-pleural nodules by using a region growingbased approach followed by an active contour refinement. Juxta-pleural lung nodules candidates are only searched inside the segmented region. Please refer to Section 3.2 ,

2. detection of nodule candidates via threshold and blob enhancement techniques. Solid nodules, which have an intensity similar to non-parenchymal tissue, are segmented by selecting an appropriate threshold. Sub-solid and non-solid nodules are first enhanced by using multiscale Laplacian-of Gaussian-filtering. Please refer to Section 3.4.

3. FP reduction via fixed rules and supervised learning. First, a reduction of FPs is performed by discarding candidates outside a given range of volume-equivalent radius. Then, a SVM classifier is trained to further reduce the number of FPs. Please refer to Section 3.5 .

Our method is developed and evaluated using the LIDC-IDRI dataset, the largest publicly available lung nodule dataset. Despite an exhaustive nodule characterization, the ground-truth of the LIDC-IDRI does not classify lung nodules according to their location as juxta-vascular, fissural, isolated and juxta-pleural. Consequently, a method for the creation of a juxta-pleural lung nodule dataset from the LIDC-IDRI is also proposed in Section 3.3 .

A schematic representation of the steps of the proposed method is shown in Fig. 3.1. Each of the steps will be detailed in the remaining of this chapter. 


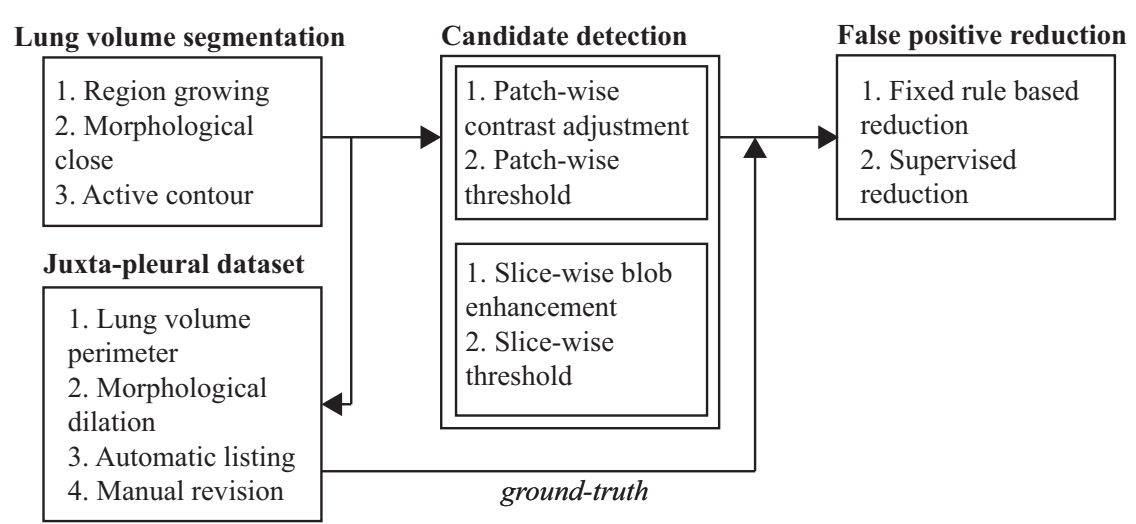

Figure 3.1: Schematic representation of the proposed method.

\subsection{Lung volume segmentation}

The proposed juxta-pleural nodule detection method searches candidates only inside the segmentation of the lung volume. Consequently, it is essential that juxta-pleural nodules are included in the lung mask. The initial lung volume segmentation is performed as proposed in Novo et al. (2014). Then, a refinement of the limits of the lung mask is performed using active contour.

\subsubsection{Initial lung volume segmentation}

Novo et al. (2014) proposed a region growing-based approach to segment the lung volume followed by morphological operations to include juxta-pleural lung nodules. Region growing is an iterative process where neighbour voxels of similar intensity are included until a given stop criteria is met. Being an intensity-based method, the contrast between the lung tissue, which is darker due to the presence of air, and the surrounding structures (Fig. 3.2a) is essential. To improve the contrast between structures, the histogram of the CT thorax is saturated for bright values. Then, a seed point on the fatty area is selected by finding the maximum intensity of the principal diagonal of one of the middle slices of the scan. Starting from this seed point, all 3D neighbour voxels whose intensity is no less than 35\% of the seed intensity are included. The seed points are then updated and the process is repeated iteratively until the segmented area remains constant. An initial lung mask is obtained by negating the computed segmentation. Due to the nature of the algorithm, lung volumes separated only by small structures may be merged. The segmentation is corrected by eroding the lung mask until 2 independent volumes exist. Finally, the segmentation is refined with a region growing of the parenchyma using a more restrict growth criteria of $10 \%$ of the seed's intensity. Note that in some slices the lung mask may be re-merged, which is not critical.

The achieved lung mask (Fig. 3.2b and Fig. 3.2c) still does not include juxta-pleural nodules. Nodule inclusion is performed using a morphological close operation with a circular structuring element of radius $15 \mathrm{~mm}$. The closing operation allows to fill the interior of the mask and include juxta-pleural nodules without altering the overall shape of the lung mask. Fig. $3.2 \mathrm{~d}$ shows the lung mask after the refinement, which now includes the juxta-pleural nodule. 


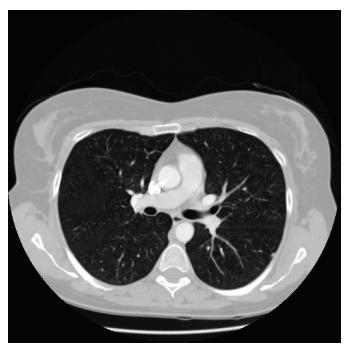

(a) Lung volume slice.

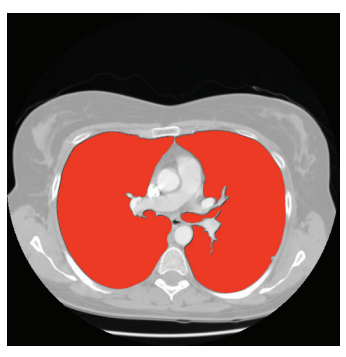

(b) Initial segmentation.

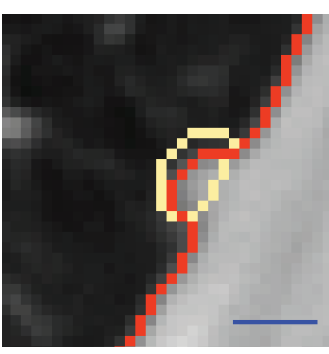

(c) Initial segmentation near nodule.

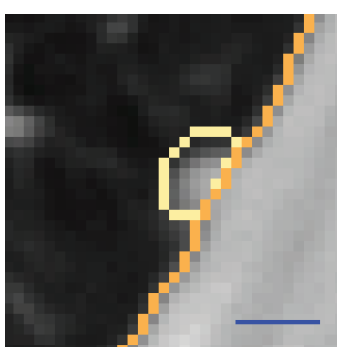

(d) Refined segmentation near nodule.

Figure 3.2: Relevant steps of the region growing-based algorithm for lung volume segmentation. The initial segmentation (prior to refinement) does not include nodules. Nodules are included after the segmentation is refined with a morphological closing. Blue scale bar corresponds to $5 \mathrm{~mm}$.

\subsubsection{Active contour refinement}

The morphological operation close preserves the overall shape of the lung volume segmentation. However, the segmentation may not be correctly adjusted to the pleura (Fig. 3.3a). A poor lung segmentation near the pleura affects the automatic detection of juxta-pleural nodules. For instance, a nodule whose diameter is smaller than the distance between the segmentation and the pleura may not be included on the mask. Consequently, the lung volume mask is corrected using a 2D ChanVese active contour approach.

The Chan-Vese segmentation, first proposed by Chan and Vese (2001), is an iterative active contour algorithm that does not rely on the image gradient to measure the system's total energy. The Chan-Vese algorithm proceeds by minimizing the segmentation function $F\left(c_{1}, c_{2}, C\right)$ :

$$
\begin{array}{r}
F\left(c_{1}, c_{2}, C\right)=\mu \cdot \text { Length }(C)+v \cdot \text { Area }(\text { inside }(C))+ \\
+\lambda_{1} \int_{\text {inside }(C)}\left|u_{0}(x, y)-c_{1}\right|^{2} d x d y+\lambda_{2} \int_{\text {outside }(C)}\left|u_{0}(x, y)-c_{2}\right|^{2} d x d y
\end{array}
$$

where $C$ is the segmentation curve, $c_{1}$ and $c_{2}$ are the average inside and outside intensities, respectively, of image $u_{0}$ and $\mu \geq 0, v \geq 0, \lambda_{1}>0, \lambda_{2}>0$ are fixed parameters that affect the evolution of the curve on each iteration. The algorithm minimizes $F\left(c_{1}, c_{2}, C\right)$ having in account the curve length, as well as the area and difference between the intensity inside and outside of the segmentation. The parameters $\lambda_{1}$ and $\lambda_{2}$ are related to the uniformity of the intensity inside and outside the curve, respectively. As suggested in Chan and Vese (2001), for simple segmentations these values can be unitary. The parameter $\mu$ is a critical parameter that allows to balance the smoothness and accuracy of the contour (Getreuer (2012)). A higher $\mu$ value results in smoother contour. The parameter $v$ directly influences the curve contraction. For the proposed application, contour smoothing rectifies some mask deformations caused by juxta-pleural nodules, increasing the volume ratio of the nodules included in the mask as demonstrated in Fig. $3.3 \mathrm{c}$ and Fig. $3.3 \mathrm{~d}$. 


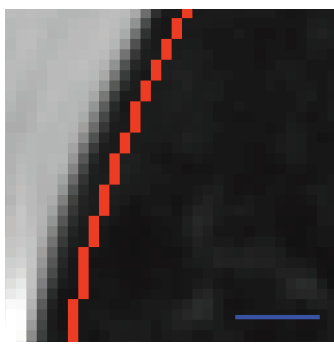

(a) After the morphological closing.

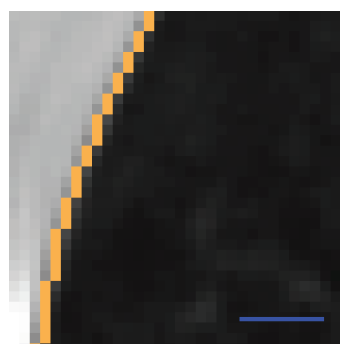

(b) (a) after the ChanVese active contour.

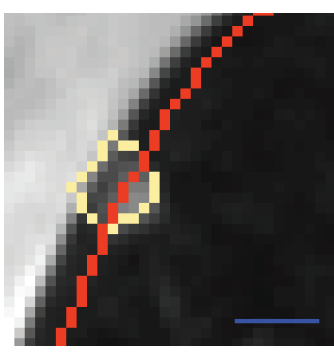

(c) After the morphological closing.

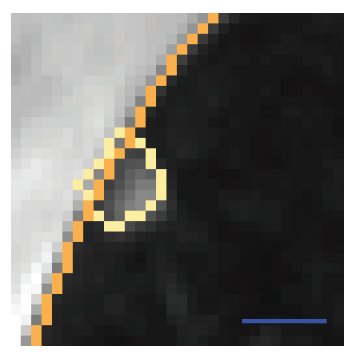

(d) (c) after the ChanVese active contour.

Figure 3.3: Details of the correction of the lung volume segmentation via the Chan-Vese active contour method. The inclusion of juxta-pleural nodules is improved (c) and d). Blue scale bar corresponds to $5 \mathrm{~mm}$.

\subsection{Dataset of juxta-pleural lung nodules}

The LIDC-IDRI dataset is the largest public dataset for the development and evaluation of CAD lung systems. However, the lack of characterization of the ground-truth in terms of nodule position i.e., isolated, juxta-vascular, juxta-pleural and peri-fissural, hinders the detailed evaluation of these systems. Due to the lack of position characterization, few works using the LIDC-IDRI report their performance for juxta-pleural nodules. In fact, most works that report detection performance for juxta-pleural nodules usually use private or small datasets where juxta-pleural nodules are identified by specialists (Retico et al. (2009); van Ginneken et al. (2010); De Nunzio et al. (2011)). The lack of a proper ground-truth on a large public available dataset hinders the comparison between detection methods. The development of a dedicated juxta-pleural lung nodule detection algorithm requires a ground-truth of juxta-pleural nodules. Consequently, a method for the creation of a dataset with the juxta-pleural nodules available in the LIDC-IDRI is created.

The computed lung volume segmentation (see Section 3.2) is used to find the juxta-pleural nodules in the LIDC-IDRI dataset. First, the perimeter of the lung mask is dilated with a disk of radius $1.5 \mathrm{~mm}$, corresponding to the smallest nodule in the dataset. The dilation compensates possible imperfections of the lung volume segmentation. All nodules of the ground-truth with at least 1 voxel overlapping the dilated perimeter are considered juxta-pleural nodules. Fig. 3.4 shows examples of nodules, with different radius, shapes and intensities, considered as juxta-pleural. The resulting dataset is then manually analyzed to remove juxta-pleural nodules erroneously considered by the initial automatic selection. All nodules that do not touch the pleural wall are removed.

Finally, the remaining juxta-pleural nodules are divided in two groups, according to their equivalent radius $r$, as small $(r<5 \mathrm{~mm})$ and large $(r \geq 5 \mathrm{~mm})$. The radius is computed as:

$$
r=\left(\frac{n_{\text {voxels }} \cdot x_{\text {scale }} \cdot y_{\text {scale }} \cdot z_{\text {scale }}}{\left(\frac{4 \pi}{3}\right)}\right)^{1 / 3}
$$

where $n_{\text {voxels }}$ is the number of voxels of the nodule, $x_{\text {scale }}, y_{\text {scale }}$ and $z_{\text {scale }}$ are the dimensions, in $m m$, of the voxels. 


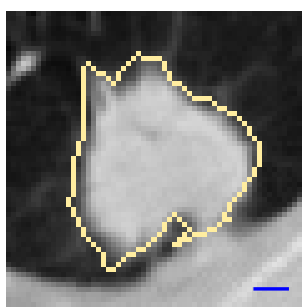

(a) 0001,91

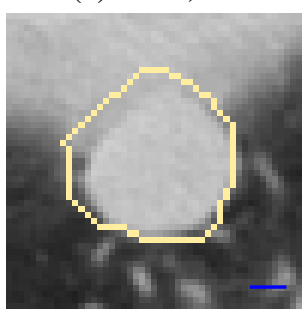

(f) 0061,40

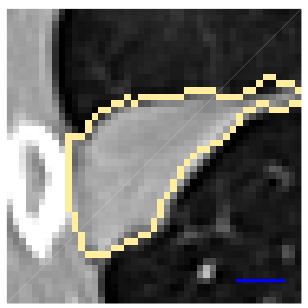

(k) 0195,87

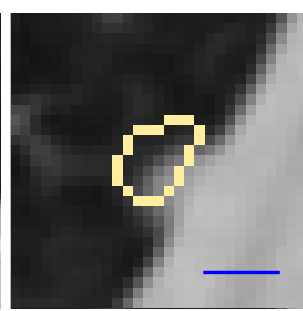

(b) 0005,89

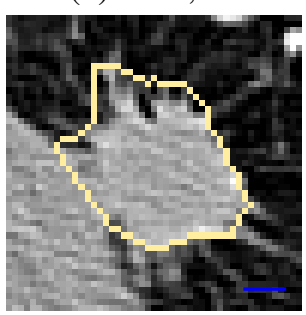

(g) 0082, 174

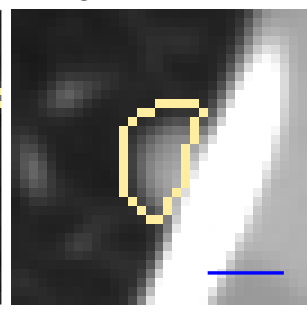

(1) 0442,43

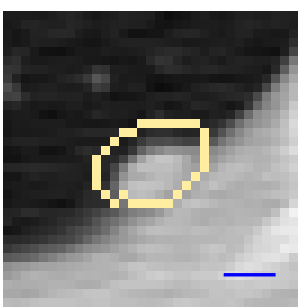

(c) 0027,113

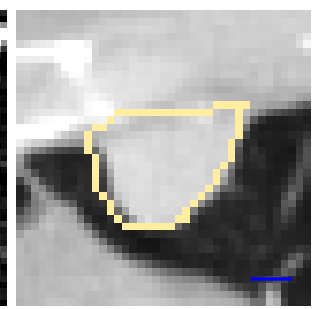

(h) 0121,161

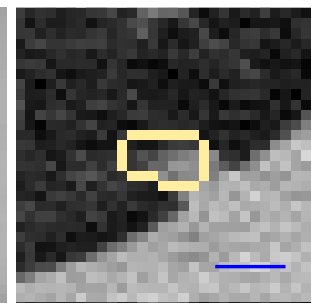

(m) 0502,53

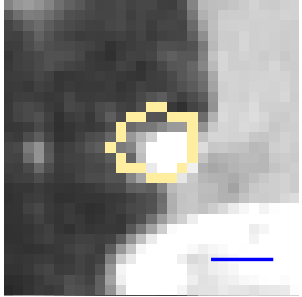

(d) 0039,134

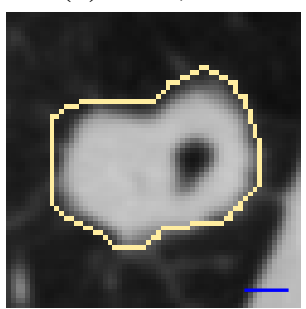

(i) 0132,28

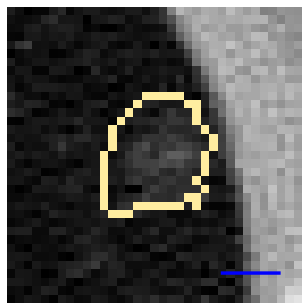

(n) 0686,196

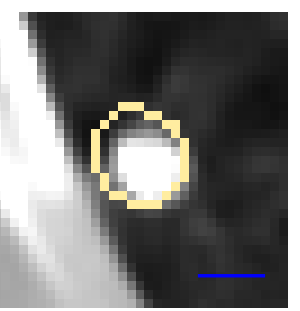

(e) 0045,63

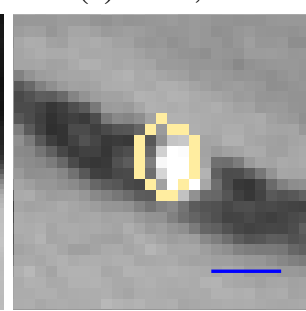

(j) 0137,31

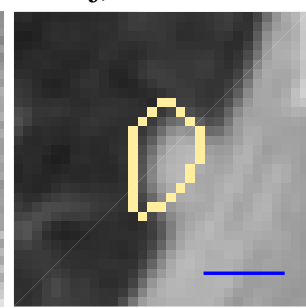

(o) 0838,175

Figure 3.4: Examples of nodules considered as juxta-pleural. Each example is retrieved from a scan and slice (LIDC-IDRI case\#, slice\#). Blue scale bar corresponds to $5 \mathrm{~mm}$.

\subsection{Candidate detection}

The proposed juxta-pleural nodule detection algorithm searches for nodule candidates inside the lung volume segmentation obtained using the method described in Section 3.2. Nodule candidates are portions of tissue that have properties similar to lung nodules. In our system, we independently search for two types of candidates: i) structures with an intensity similar to non-parenchymal tissue and ii) small blobs, which are rounded structures with an intensity higher than the surrounding background. These two characteristics, intensity and blobness, exploit the natural rounded structure and the intensity similarity between the non-parenchymal tissue and the majority of the juxta-pleura nodules. Intensity-related candidates are found by selecting an appropriate threshold value over a given slice scan, as explained in Section 3.4.1. On the other hand, blob candidates are enhanced prior to detection by using a blob enhancement technique, as detailed in Section 3.4.2.

\subsubsection{Direct threshold}

The majority of the juxta-pleural nodules in the LIDC-IDRI has solid texture. Solid nodules have an intensity similar to the non-parenchymal tissue (Retico et al. (2008); De Nunzio et al. (2011)). Consequently, the selection of an appropriate intensity threshold allows to obtain a binary 


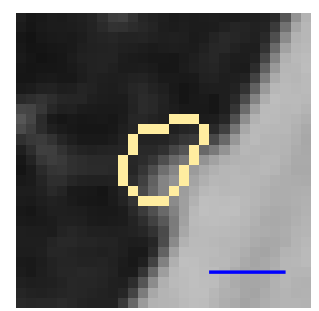

(a) $(0005,89)$

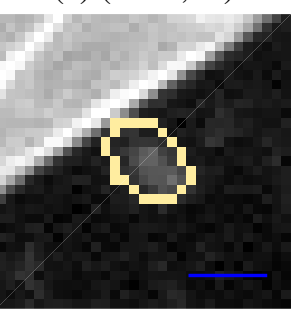

(f) $(0620,91)$

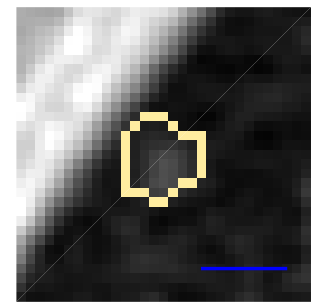

(k) $(0821,372)$

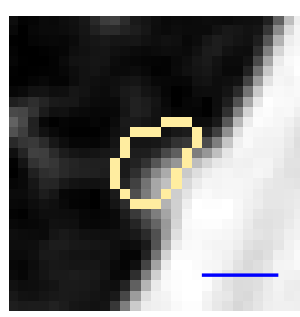

(b) Saturated.

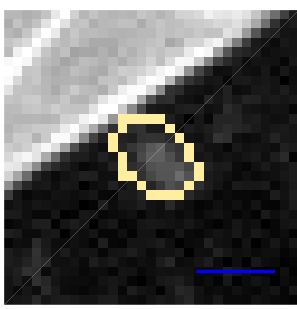

(g) Saturated.

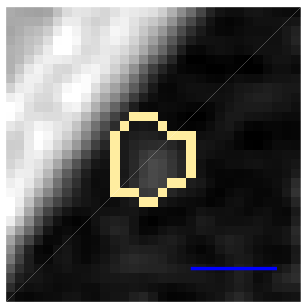

(1) Saturated.

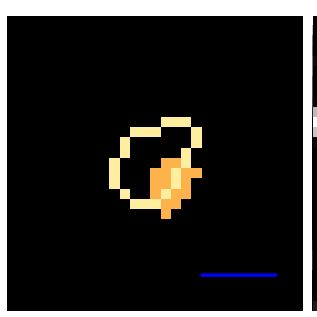

(c) b after threshold.

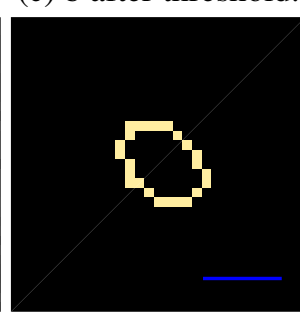

(h) g after threshold.

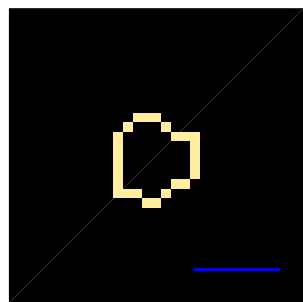

(m) 1 after threshold.

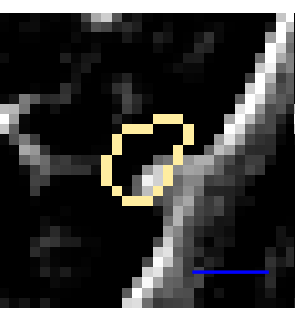

(d) Blob enhanced.

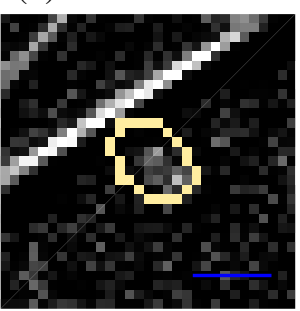

(i) Blob enhanced.

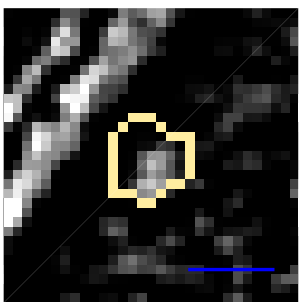

(n) Blob enhanced. (o) n after threshold.

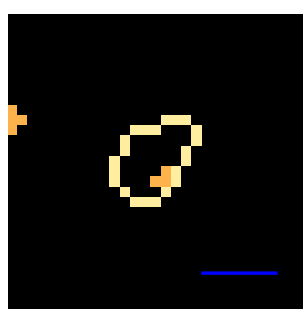

(e) d after threshold.

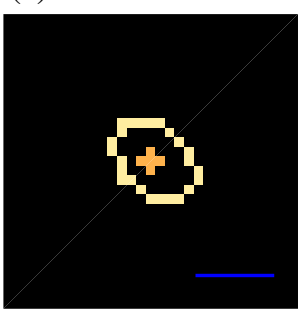

(j) i after threshold.

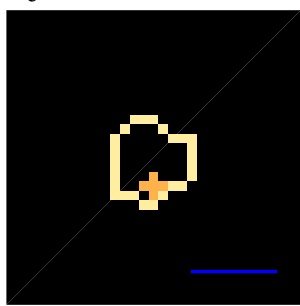

Figure 3.5: Steps for juxta-pleural nodule detection. (a)-(d): solid nodule; (f)-(i): sub-solid nodule; (k)-(n): non-solid nodule. For the blob enhancement step, Laplacian-of-Gaussian filters with $\sigma=\{1,1.5,2\}$ are used. Each example is retrieved from a scan and slice (LIDC-IDRI case\#, slice\#). Blue scale bar corresponds to $5 \mathrm{~mm}$.

mask of the candidate. The threshold selection is performed for each slice of the volume and inside a sliding $30 \times 30 \mathrm{~mm}$ patch. This patch size allows to have a representative occurrence of both parenchymal and non-parenchymal tissue, easing the threshold selection. First, contrast is increased by saturating $1 \%$ of the intensity values at low and high intensities of the current patch. The first and second columns of Fig 3.5 show patches with nodules before and after the contrast adjustment. Note that while the contrast between non-parenchymal and parenchymal tissue is increased, sub-solid and non-solid nodules remain with little contrast. This problem will be partially solved by the blob enhancement process (Section 3.4.2).

Finally, the patch is threshold using the value that maximizes the inter-class distance of the intensity histogram (Otsu (1979)). The result of the threshold is combined with the lung mask using the logical operation AND. Fig. 3.5 shows examples of this process. Note that using the direct threshold method only the solid nodule is detected. 


\subsubsection{Blob enhancement}

Sub-solid and non-solid nodules have low contrast with the parenchymal tissue. As a consequence, the contrast between these nodules and the surrounding tissue must be enhanced prior to their detection. This process is referred as blob enhancement. In our work, a multi-scale Laplacian-ofGaussian (LoG) approach is used. LoG filtering is a blob-enhancement technique vastly used for the detection of rounded structures, such as the majority of the lung nodules. Also, multi-scale approaches are advantageous over single scale because they allow to combine blob-like structures of different radius (Kong et al. (2013); El-Baz et al. (2013); Valente et al. (2016)). The LoG filter corresponds to the second derivative of the Gaussian function $G(x, y, \sigma)$ and it is defined as:

$$
-\nabla^{2} G(x, y, \sigma)=-\frac{x^{2}+y^{2}-2 \sigma^{2}}{2 \pi \sigma^{6}} \cdot e^{-\frac{x^{2}+y^{2}}{2 \sigma^{2}}}
$$

where $\sigma$ controls the radius and amplitude of the LoG filter (Marr and Hildreth (1980)). Fig. 3.6 shows examples of LoG filters for different $\sigma$ values. Note that the filter's amplitude is affected by a factor of $\sigma^{-4}$.

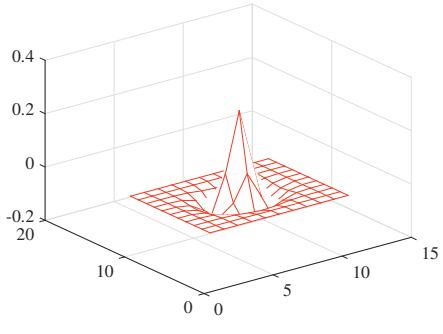

(a) $\sigma=1$

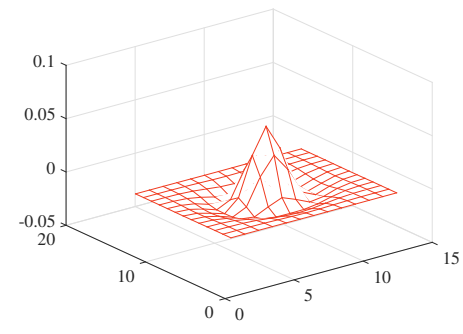

(b) $\sigma=1.5$

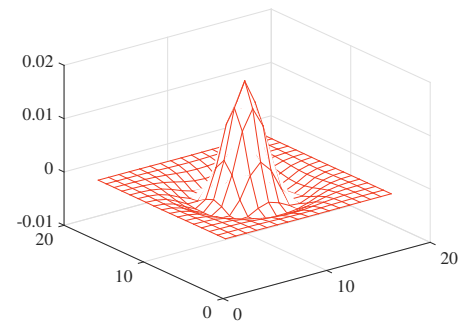

(c) $\sigma=2$

Figure 3.6: Examples of Laplacian of Gaussian blob enhancement filters for different $\sigma$ values.

For each slice of the volume, $I$, the image is enhanced by convolving with LoG filters of different $\sigma$ values. The maximum response for each filter is then combined:

$$
\sigma_{1}<\sigma<\sigma_{i}\left(I *\left[-\nabla^{2} G(x, y, \sigma)\right]\right)
$$

After the enhancement using Eq. 3.4, the optimal threshold is determined inside the lung mask by using the method proposed by Otsu (1979). Please note that, in the proposed approach, the different LoG filters are not normalized before the operation described in Eq. 3.4 is performed. This means that structures with smaller diameters (approximately 5 voxels slice-wise or $7 \mathrm{~mm}$ if $\sigma=1$ ) are heavily prioritized over to larger structures. This approach allows to enhance the most noticeable portion of sub-solid and non-solid nodules. Although this process does not allow a clean segmentation of the entire structure (Fig. 3.5), it was found that it significantly reduces both the number FP and risk of connection between structures. The connection between structures hinders the FP reduction step, greatly affecting the overall sensitivity of the system. Consequently, it 
was considered more relevant to under-segment sub-solid and non-solid nodules without compromising the detection of solid nodules than the opposite.

The final candidate mask is obtained combining the two candidate masks (direct threshold and blob enhancement) with a logic OR operation. Each nodule candidate corresponds to a 3D connected component considering a 26 voxel neighbourhood connectivity. At this stage, a candidate is considered a TP if at least 1 voxel overlaps the ground-truth. Otherwise it is considered a FP.

\subsection{False positive reduction}

The candidate detection step results on a high number of FPs. These FPs correspond mainly to blood vessels of different calibers because of the intensity and blobness similarity with lung nodules. A system with high number of FPs is not of practical use for physicians. Furthermore, FP reduction should be performed in such way that the maximum number possible of TPs are preserved. In our method, FP are reduced in two steps: first, fixed rules are applied to remove all candidates that obviously are not nodules; then, a SVM is trained to perform a refined reduction.

\subsubsection{Fixed-rules based FP reduction}

The proposed algorithm is designed to detect nodules with equivalent radius $\leq 5 \mathrm{~mm}$. Also, the radius of the smallest segmented nodule of the LIDC-IDRI dataset is $1.5 \mathrm{~mm}$. Candidate radii is computed using Eq. 3.2. Assuming a safety margin due to possible connection between different candidates, all candidates with radius $\geq 6 \mathrm{~mm}$ are removed. Juxa-pleural candidates are filtered by eliminating those that do not have at least 1 voxel at a $6 \mathrm{~mm}$ distance from the perimeter of the lung volume segmentation i.e., by eliminating all candidates that have no voxels on a $6 \mathrm{~mm}$ radius disk dilation of the lung mask perimeter. All candidates with radius $<1.5 \mathrm{~mm}$, corresponding to the smallest nodule of the LIDC-IDRI, are also removed.

\subsubsection{Supervised learning FP reduction}

FPs from the candidate set are further reduced by using supervised learning. SVM with radial basis function kernel (SVM-rbf) is used because it as shown good FP-reduction capabilities (de Carvalho Filho et al. (2014); Valente et al. (2016)). SVMs operate by maximizing the margin between two classes on a given feature space, i.e by maximizing the distance on the feature plane between a given set of observations of different classes and the decision hyperplane, while reducing the number of misclassifications. The samples that define the margin are referred as support vectors. A cost parameter $C$ is introduced to enforce a soft-margin on the classifier, ensuring a trade-off between high margins and misclassification. 
Table 3.1: Features used for supervised learning.

\begin{tabular}{llll} 
No & Category & Location & Features \\
\hline $1-5$ & Intensity & Candidate & mean, standard deviation, entropy, skewness, kurtosis \\
$6-10$ & & Bounding box & mean, standard deviation, entropy, skewness, kurtosis \\
$11-15$ & & Outside & mean, standard deviation, entropy, skewness, kurtosis \\
\hline $16-20$ & Blobness & Candidate & mean, standard deviation, entropy, skewness, kurtosis \\
$21-25$ & & Bounding box & mean, standard deviation, entropy, skewness, kurtosis \\
$26-30$ & & Outside & mean, standard deviation, entropy, skewness, kurtosis \\
\hline $31-35$ & Gradient & Candidate & mean, standard deviation, entropy, skewness, kurtosis \\
$36-40$ & & Bounding box & mean, standard deviation, entropy, skewness, kurtosis \\
$41-45$ & & Outside & mean, standard deviation, entropy, skewness, kurtosis \\
\hline 46 & Geometric & Candidate & maximum $2 \mathrm{D}$ axis length slice-wise $(\mathrm{mm})$ \\
47 & & Candidate & volume $\left(\mathrm{mm}^{3}\right)$ \\
48 & & Candidate & sphericity \\
49 & & Candidate & compactness 1 = volume/(bounding box volume) \\
50 & & Candidate & compactness 2 = volume/(bounding cube volume) \\
\hline 51 & Distance & Candidate & mean value of the lung mask distance transform
\end{tabular}

Instead of measuring this distance using a scalar product, the RBF kernel is used since it allows for a higher degree of generalization of the hyperplane:

$$
K\left(x_{1}, x_{2}\right)=e^{-\gamma \cdot d\left(x_{1}-x_{2}\right)}
$$

where $x_{1}$ and $x_{2}$ are two observations, $d$ is the euclidean distance between the samples and $\gamma$ is the parameter that controls the range of influence of each support vectors (Pedregosa et al. (2011); Yu-Jen Chen et al. (2015)).

The classifier is trained with features inspired by the works of Murphy et al. (2009); De Nunzio et al. (2011); Han et al. (2015). The features used in our work are presented in Table 3.1

The features referred in Table 3.1 are collected from the candidate volume, the candidate bounding box volume and the volume $6 \mathrm{~mm}$ outside the candidate. Namely, the volume outside the candidate is computed by performing morphological dilation followed by the logic AND operation with the negation of the candidate. A detailed explanation of some of the features is given next.

Hessian-based blobness features are computed according to Li et al. (2003) considering $\sigma=$ $\{0.5,1.5,3\}$. Entropy is a measure of the randomness of the volume and it is defined as

$$
\text { entropy }=-\sum_{i=1}^{256}\left(p_{i} \cdot \log _{2}\left(p_{i}\right)\right)
$$

where $p$ is the histogram count of the bin $i$. Skewness and kurtosis are the third and fourth moments and indicate how assymetric and how tayled a given distribution is, respectively. These metrics 
are described as

$$
\text { moment }=\frac{\sum_{i=1}^{N}\left(x_{i}-\bar{x}\right)^{n}}{N \cdot \sigma^{n}}
$$

where $N$ is the number of voxels, $x_{i}$ is the intensity for voxel $i$ and $\bar{x}$ is the mean intensity of the candidate; $n=3$ is used for skewness and $n=4$ for kurtosis (Glass and Hopkins (1996)). Sphericity is the degree of overlap of the candidate with a sphere of equivalent radius using the Dice's coefficient (Dice (1945))

$$
\text { sphericity }=\frac{2(\text { candidate } \cap \text { sphere })}{N+S}
$$

where $N$ and $S$ are the number of voxels of the candidate and sphere, respectively.

The SVM-rbf classifier is retrieved from Chang and Lin (2011). The features' values from all the candidates in the dataset of juxta-pleural nodules with radius $\leq 5 \mathrm{~mm}$ are concatenated in a single feature table. Each row of the table corresponds to a candidate $i$, whereas each column corresponds to a given feature $j$. Candidates with at least 1 voxel overlapping the ground-truth are considered as TP. During the learning process, the feature table is randomly divided in training and test sets. This division is performed scan-wise, so that candidates from the same scan are not in the training and test sets simultaneously. The training set is normalized by using the median absolute deviation, due to the presence of outliers:

$$
\begin{gathered}
x_{i j_{\text {normalized }}}=\frac{x_{i j}-\operatorname{median}\left(X_{j}\right)}{M A D} \\
M A D=\operatorname{median}\left(\left|X_{j}-\operatorname{median}\left(X_{j}\right)\right|\right)
\end{gathered}
$$

where $x_{i j}$ is the value of feature $j$ for the training candidate $i$ and $X_{j}$ is a vector with all the values of feature $j$ considering only the training set. The median and MAD values used to normalize the training set are then used to normalize the test set. The training and test processes are repeated a fixed number of times. The mean classifier behaviour is obtained by averaging the classifier performance during the repetitions. The $C$ and $\gamma$ parameters of the classifier are determined via grid-search over the entire normalized candidate set using 5-fold cross validation. The studied $C$ values are $\left\{2^{-5} \ldots 2^{15}\right\}$ and the $\gamma$ values are $\left\{2^{-15} \ldots 2^{3}\right\}$, (Chang and Lin (2011)). 


\subsection{Performance evaluation}

The performance of the proposed CADe system is evaluated in terms of sensitivity and FPs produced. Sensitivity corresponds to the ratio of correct detections:

$$
\text { sensitivity }=\frac{T P}{T P+F N}
$$

where $T P$ is the number of true positives and $F N$ the number of false negatives (missed nodules). In CADe systems, sensitivity varies with the number of FP/scan. Free-Response receiver operating characteristic (FROC) curve plots sensitivity as function of the number of FP/scan. Systems can be evaluated by selecting a point from the FROC curve. However, it is hard to define a proper balance between high sensitivity and low FPs. Consequently, van Ginneken et al. (2010) proposed a score metric for the evaluation of CAD systems. This score averages the sensitivity for different numbers of FPs per scan:

$$
\text { score }=\frac{\sum_{i=-3}^{3} F_{R O C_{\text {system }}}\left(2^{i}\right)}{7}
$$

The FROC curve can be derived from the receiver operating characteristic (ROC) curve and plots the sensitivity as function of the number of FP. The ROC curve plots the TP rate of the system as function of the FP ratio:

$$
F P_{\text {ratio }}=\frac{F P}{F P+T N}
$$

where $T N$ is the number of true negatives.

ROC curves can be obtained by varying the decision threshold for the positive class. For instance, ROC curves are easily computed during the classification process by varying the decision criteria between classes. Furthermore, the area under the ROC curve (AUC) is a good metric of the system performance (Flach et al. (2011)). A higher AUC implies that the system is able to achieve higher sensitivities without a significant increase of the FP ratio. In this work, AUC is used to compare the performance of the supervised FP reduction with other works.

The FROC curve of the system is computed from the ROC curve of the classifier:

$$
F R O C_{\text {system }}\left(F P_{\text {scan }}\right)=\text { sensitivity }_{\text {prior classification }} \cdot R O C_{\text {classifier }}\left(\frac{F P_{\text {scan }}}{\overline{F P_{\text {scan }}}}\right)
$$

where $F P_{\text {scan }}$ is the number of FPs per scan, $\overline{F P_{\text {scan }}}$ and sensitivity prior classification $_{\text {is the average }}$ number of FPs and is the maximum achieved sensitivity prior to classification, respectively. 


\subsection{Concluding remarks}

A method for the detection of small juxta-pleural lung nodules in CT scans is presented. Our method exploits both the intensity similarity between nodules and non-parenchymal tissue and the natural roundness of these abnormalities. The LIDC-IDRI has no ground-truth for juxta-pleural nodules and thus a dataset with this type of nodules is created.

The candidates are searched inside a lung volume segmentation properly rectified with an active contour-based approach to include juxta-pleural nodules. The active contour rectification is expected to behave better than morphological operations over the lung mask since it considers local intensity information and contour shape instead of only shape.

Candidate detection is performed via direct threshold and blob enhancement. Direct threshold is expected to perform a proper segmentation of solid nodules due to their high contrast with the parenchyma. On the other hand, the identification of sub-solid and non-solid is more challenging due to the lack of contrast of these structures and is thus achieved through blob enhancement with multi-scale Laplacian-of-Gaussian filtering.

The number of FPs is reduced via fixed-rules and supervised learning. Fixed-rules are essential prior to the classifier-based reduction because our method produces a high number of FPs. It is, thus, a crucial step that controls the performance of the algorithm. The features and classifier selected for the supervised learning are based on state-of-the-art works and should significantly reduce the number of FPs produced by our system. 


\section{Chapter 4}

\section{Results and discussion}

This chapter presents and discusses the results of each of the steps explained in Chapter 3 . We discuss the results on the juxta-pleural nodule dataset, lung volume segmentation, candidate detection and FP reduction. A comparison with the state-of-the-art is also performed. We analyze methods dedicated to the detection of juxta-pleural lung nodules, namely Retico et al. (2008) and De Nunzio et al. (2011). Our results are also compared with Han et al. (2015) as they state juxtapleural detection performance in the LIDC-IDRI dataset. A final discussion including the research of van Ginneken et al. (2010) is also performed because, even though the LIDC-IDRI dataset is not used, it presents a proper performance assessment for juxta-pleural nodules. Please refer to Section 2.2 and Section 2.3 for a description of their research.

\subsection{Dataset of juxta-pleural nodules}

A dataset with the existing juxta-pleural nodules of the LIDC-IDRI is herein presented. To the best of our knowledge, this juxta-pleural lung nodule dataset is the first to be collected from a large portion of the LIDC-IDRI. We have analyzed 729 out of the 1012 available scans. The remaining scans were not considered due to reading errors of either. $\mathrm{dcm}$ or. $\mathrm{xml}$ files. The same problems had already been reported by Murphy et al. (2009), although these authors managed to use 888 scans. The difference in the number of scan read might be due to the reading protocol used.

Furthermore, our dataset is manually revised to remove mislabelled nodules i.e., nodules that were erroneously considered as juxta-pleural because they were partially contained in a lung perimeter dilation using a disk of radius $1.5 \mathrm{~mm}$. Similarly, in Han et al. (2015) the authors used a subset of the LIDC-IDRI and reported their system performance for juxta-pleural nodules. A nodule was considered juxta-pleural if at least 1 voxel was contained on a 5-layer erosion of the lung volume segmentation. However, a manual revision was not performed and thus nodules that did not contact with the pleura were most likely included. For instance, Table 4.1 shows the number of scans and juxta-pleural nodules considered before and after the manual revision, thus showing the importance of this step. The number of juxta-pleural nodules is reduced by $11 \%$ after the manual revision. Fig. 4.1 shows examples of manually removed nodules. 
Table 4.1: Number of scans and juxta-pleural nodules in the studied 729 scans from the LIDCIDRI dataset. Small juxta-pleural nodules are those with radius $\leq 5 \mathrm{~mm}$.

\begin{tabular}{lcc} 
& No. Scans & No. juxta-pleural nodules \\
\hline Before manual revision & 469 & 837 \\
After manual revision & 432 & 747 \\
\hline Small juxta-pleural nodules & 315 & 510 \\
\hline
\end{tabular}

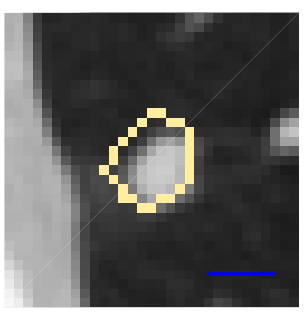

(a) 0042, 43

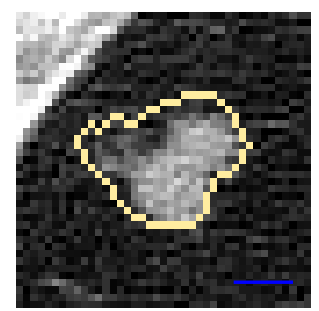

(f) 0415,132

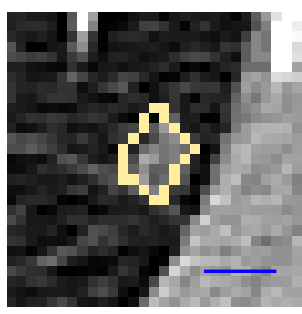

(b) 0131,123

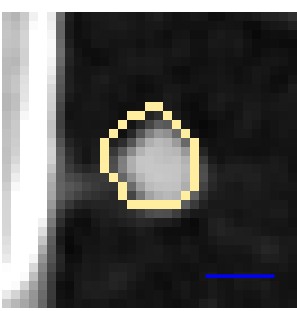

(g) 0655,210

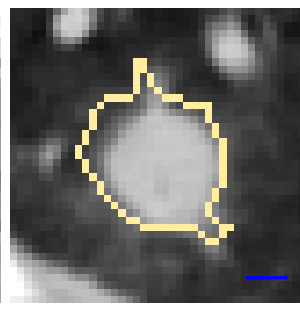

(c) 0171,21

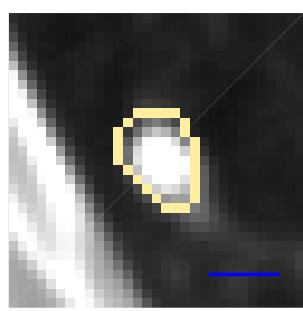

(h) 0713,83

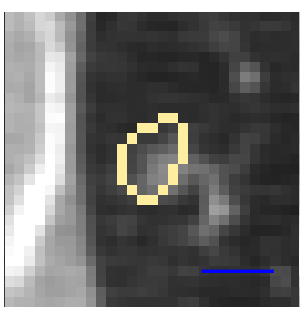

(d) 0176,167

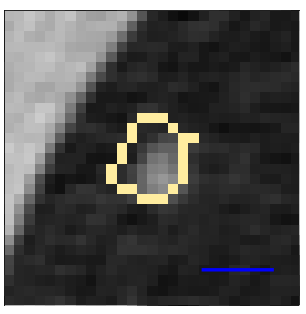

(i) 0858,218

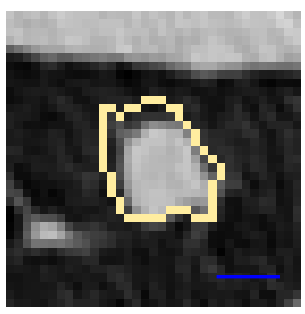

(e) 0300,182

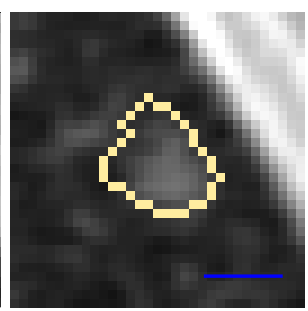

(j) 0896,30

Figure 4.1: Examples of nodules removed from the juxta-pleural dataset. Each example is retrieved from a scan and slice (LIDC-IDRI case\#, slice\#). Blue scale bar corresponds to $5 \mathrm{~mm}$.

Despite not having position-related labels, the LIDC-IDRI characterizes nodules using different metrics. For instance, the agreement level corresponds to the number of specialists that were in accordance if a given abnormality is, in fact, a nodule. Empirically, a lower agreement level is indicative of a higher detection difficulty. Similarly, the subtlety of the nodule is a direct indicator of how difficult a nodule is to be identified by humans. Nodule texture, i.e., nodule classification as non-solid, sub-solid or solid, is also of interest. For instance, whereas solid nodules are highly contrasted in relation to the parenchyma, non-solid nodules lack contrast and thus require more effort to be detected (Armato et al. (2011); Setio et al. (2015)). Some statistics of interest of both juxta-pleural and non-juxta-pleural nodules of the LIDC-IDRI dataset are presented in Fig. 4.2.

According to Fig. $4.2 \mathrm{a}$ and $4.2 \mathrm{~b}$, the nodule radius distribution for the all nodules of the LIDCIDRI dataset and the existing juxta-pleural nodules is similar. Approximately $65 \%$ of the juxtapleural nodules have a radius $\leq 5 \mathrm{~mm}$. The design of strategies for small nodules is of interest because they occur more frequently than larger nodules. Furthermore, the agreement level for small juxta-pleural nodules tends to reduce significantly in comparison to the majority of the nodules, as shown in Fig. 4.2c The lack of agreement indicates that these abnormalities are hard to distinguish from small blood vessels and other aberrations that occur near the pleura. This is, in fact, corroborated by the higher percentage of small juxta-pleural nodules in the second tier 


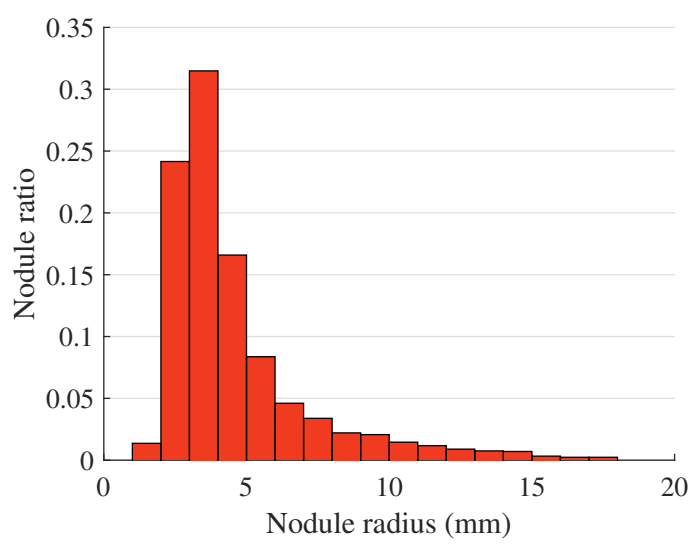

(a) LIDC-IDRI nodule radius distribution.

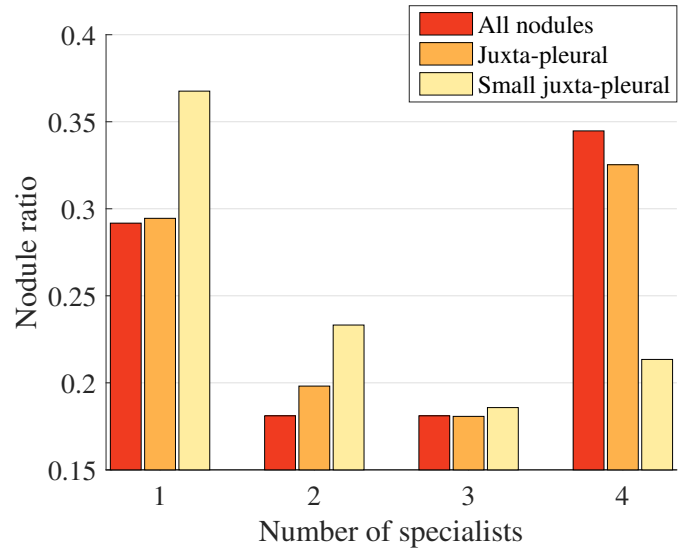

(c) Nodule agreement level.

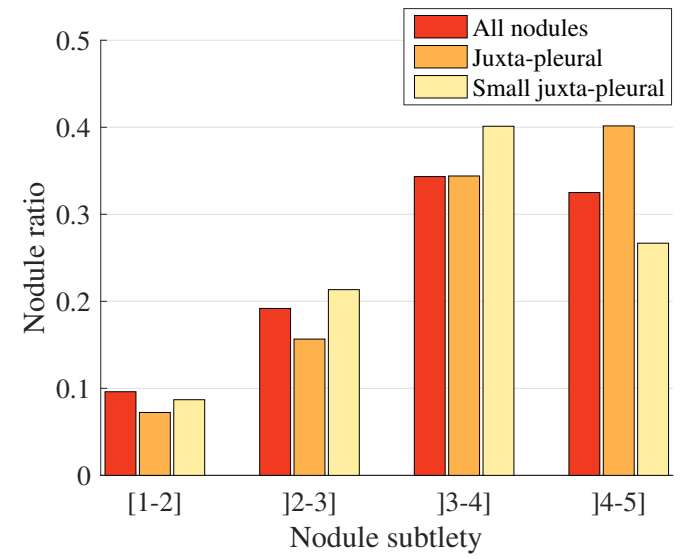

(e) Nodule subtlety distribution.

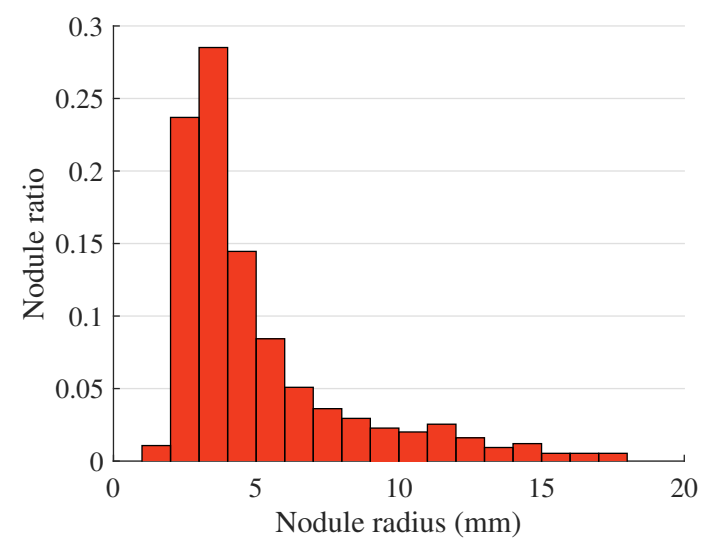

(b) Juxta-pleural radius distribution.

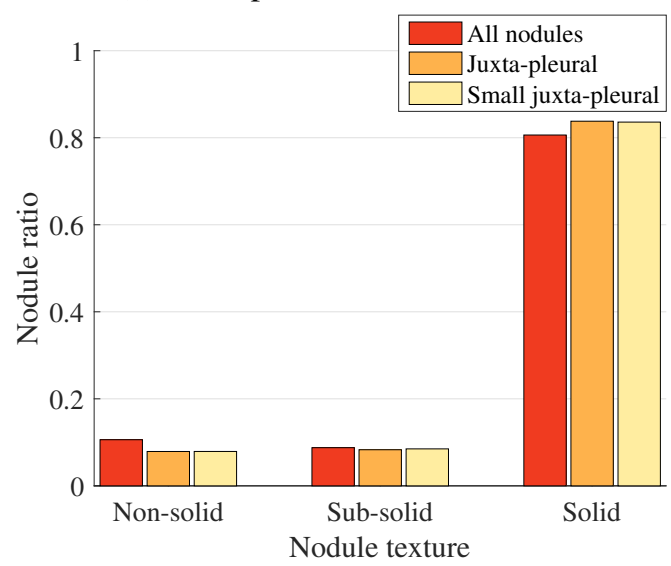

(d) Nodule texture distribution.

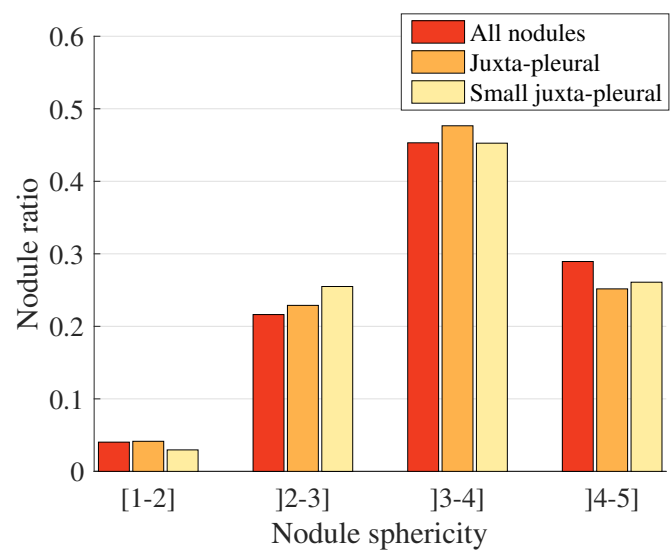

(f) Nodule sphericity distribution.

Figure 4.2: Statistics retrieved from the LIDC-IDRI, with special focus on juxta-pleural nodules. Small juxta-pleural nodules have radius $\leq 5 \mathrm{~mm}$. 


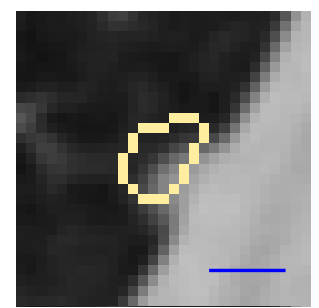

(a) 0005,89

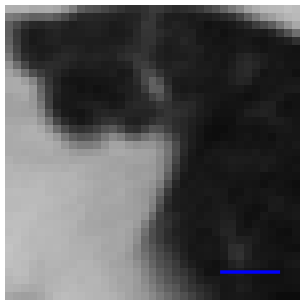

(f) 0005,50

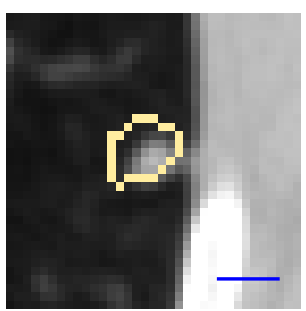

(b) 0034,98

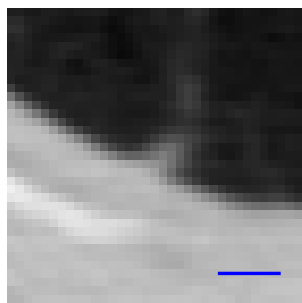

(g) 0034,176

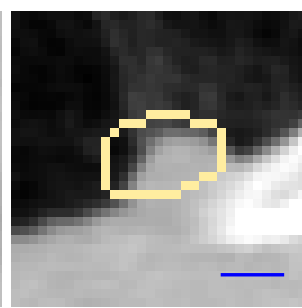

(c) 0529,268

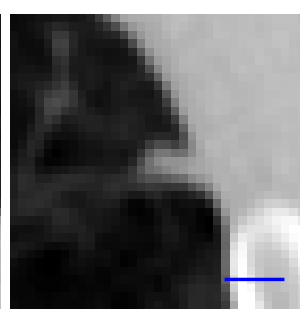

(h) 0529, 391

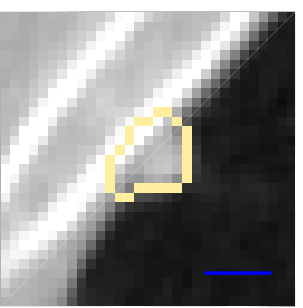

(d) 0854,174

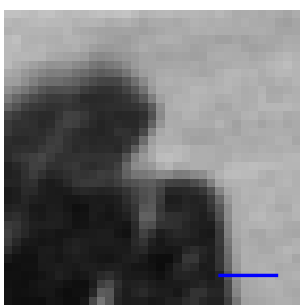

(i) 0854,130

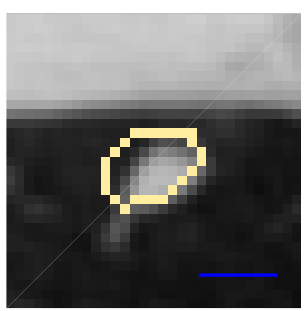

(e) 0936,217

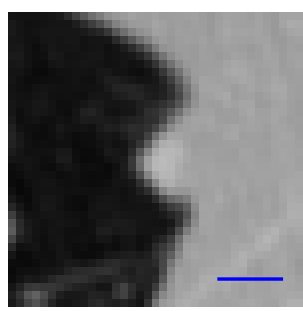

(j) 0936,168

Figure 4.3: Examples of juxta-pleural lung nodules with radius $\leq 5 \mathrm{~mm}$ (first row) and non-nodule structures (second row). Each example is retrieved from a scan and slice (LIDC-IDRI case\#, slice\#). Blue scale bar corresponds to $5 \mathrm{~mm}$.

subtlety group in comparison to the first tier (]4-5]). In this scale the obviousness of the nodule increases with the subtlety value. Finally, the lower sphericity of the nodules in study indicate that blob detectors are not as efficient for the enhancement of these structures, further hindering the detection of sub-solid and specially non-solid nodules. Fig. 4.3 shows examples of juxta-pleural nodules and other structures that despite not being nodules have similar visual features.

Juxta-pleural nodules tend to have characteristics, namely low agreement level, difficult position and high subtlety, that increase their detection difficulty in comparison to other nodules. With this in consideration, it is understandable why these nodules tend to have a lower detection sensitivity. From this point forward, we consider the juxta-pleural nodule dataset as containing only nodules with radius $\leq 5 \mathrm{~mm}$ because they are the most challenging to detect. This dataset contains approximately $68 \%$ of the considered juxta-pleural nodules (i.e., 510 out of 747 nodules).

\subsection{Lung volume segmentation}

A proper lung volume segmentation is essential for our juxta-pleural nodule detection method because the candidates are only searched inside the segmented region. After the initial segmentation using region growing, our active contour model is initialized with $\lambda_{1}=\lambda_{2}=\mu=1$. $v$ is set to -0.4 to incentivize the contour to grow outwards and thus compensate the initial segmentation.

Note that there is no ground-truth available for the lung volume segmentation. Consequently, and having in account the objective of our method, the evaluation is performed in terms of the average volume (or portion) of juxta-pleural nodules included by the segmentation. This nodule inclusion volume is computed by performing a logical AND operation between the lung volume segmentation and the juxta-pleural nodule ground truth. Then, the volume ratio between this 
Table 4.2: Inclusion of juxta-pleural nodules and juxta-pleural nodules with radius $\leq 5 \mathrm{~mm}$ by the segmented lung volume.

\begin{tabular}{ccc} 
& Novo et al. $\mathbf{~ ( 2 0 1 4 )}$ & Proposed method \\
\hline Inclusion volume (\%) & $80.0 \pm 26.1$ & $86.5 \pm 20.7$ \\
Included nodules (\%) & 93.9 & 98.0 \\
\hline Inclusion volume (radius $\leq 5 \mathrm{~mm}, \boldsymbol{\%})$ & $77.4 \pm 27.9$ & $86.2 \pm 21.4$ \\
Included nodules (radius $\leq 5 \mathrm{~mm}, \boldsymbol{\%})$ & 92.2 & 97.8 \\
\hline
\end{tabular}

intersection and the ground-truth from the dataset of juxta-pleural nodules with radius $\leq 5 \mathrm{~mm}$ is computed. This inclusion volume is compared before, i.e. as proposed by Novo et al. (2014), and after the correction of the lung volume segmentation using the Chan-Vese active-contour.

Table 4.2 shows that the proposed lung volume segmentation correction method improves the inclusion volume of small juxta-pleural nodules by approximately 10\%. For these statistics, nodules with less than $25 \%$ inclusion volume are not considered as included by the lung mask because the great majority of the nodule is outside the volume segmentation. Results prove that the active contour approach is advantageous when used to complement the morphological closing. Note that active contours have in consideration the local intensity of the image for the adjustment of the contour and, consequently, are able to perform shape corrections locally. On the other hand, the used morphological operations only consider the binary mask and thus do not have enough information to properly adjust to the lungs' limits.

Despite increasing the inclusion volume, which eases candidate detection, there are still nodules that are not included by the lung mask (Fig. 4.4). These nodules are difficult to include because they appear near vertexes of the lungs. There, the nodules act like a barrier that both region growing and active contours fail to properly identify. In fact, all nodules that are not included in the lung volume segmentation have solid texture. This is to be excepted because unlike subsolid and non-solid nodules, solid nodules significantly deform the pleural region and thus affect the lung volume segmentation. A possible solution would be to use a 3D model of an average lung mask as the starting point for a 3D active contour-based approach. Considering Table 4.2, the maximum sensitivity of our system for juxta-pleural nodules with radius $\leq 5 \mathrm{~mm}$ is $97.8 \%$.

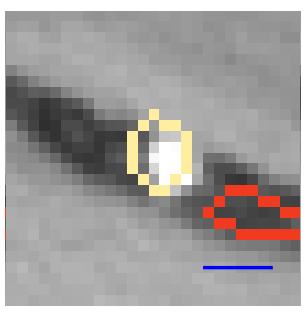

(a) 0137,31

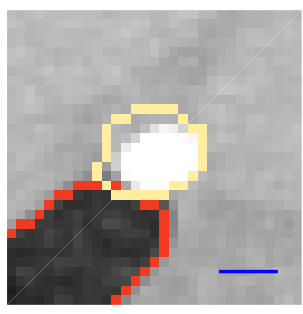

(b) 0158,54

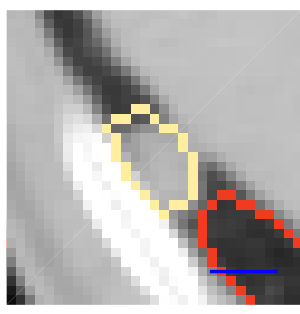

(c) 0198,31

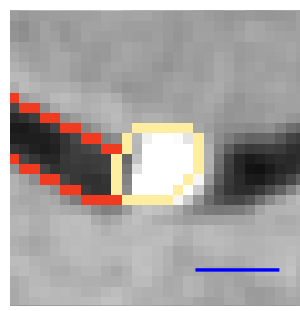

(d) 0634,174

Figure 4.4: Examples of nodules not included in the lung volume segmentation due to their location near the limits of the lungs. Each example is retrieved from a scan and slice (LIDC-IDRI case\#, slice\#). Blue scale bar corresponds to $5 \mathrm{~mm}$. 
Table 4.3: Maximum achieved sensitivity values (\%) for the proposed method, prior to the false positive reduction step, per nodule texture and minimum agreement level between specialists.

\begin{tabular}{ccccc} 
& \multicolumn{4}{c}{ Nodule texture } \\
\cline { 2 - 5 } Agreement level & Non-solid & Sub-solid & Solid & Overall \\
\hline At least 4 & 60.0 & 100 & 99.0 & 97.2 \\
At least 3 & 42.9 & 100 & 97.8 & 96.0 \\
At least 2 & 40.0 & 100 & 96.7 & 94.4 \\
At least 1 & 55.0 & 97.7 & 95.0 & 92.1 \\
\hline Number of nodules & 42 & 45 & 423 & 510 \\
\hline
\end{tabular}

\subsection{Candidate detection}

Our juxta-pleural detection method searches for candidates inside the lung volume segmentation. Because of that, juxta-pleural nodules that are not properly included in the lung mask are not identified by our system. As referred in Section 4.2, this situation implies a lack of inclusion of some juxta-pleural nodules with radius $\leq 5 \mathrm{~mm}$, corresponding to an overall maximum achievable sensitivity of $97.8 \%$. In our method, solid nodules are detected via direct threshold after a contrast adjustment. Sub-solid and non-solid nodules are enhanced as described in Section 3.4.2 considering $\sigma=\{1,1.5,2\}$. This $\sigma$ range corresponds to blobs with a diameter approximately between 5 and 12 voxels, or a candidate radii range between $3.5 \mathrm{~mm}$ and $8.5 \mathrm{~mm}$. Please recall that small structures are heavily prioritized over the larger ones, which is the reason why we allow the larger LoG radius to exceed the maximum nodule radius in the dataset. The slice-wise candidate search and the prioritization of small structures by the LoG filters reduces the segmentation performance of the method. For instance, due to the partial-volume effect, small solid nodules are usually only well circumscribed and contrasted on a single slice, hindering the detection of the same candidate on adjacent slices. Smooth boundaries between the lung and the non-parenchymal tissue further affect the segmentation. Furthermore, the ground-truth sometimes does not start exactly attached to the lung wall, as visible in Fig. $4.5 \mathrm{c}$ and Fig. $4.5 \mathrm{~d}$, and thus our segmentation includes volumes outside the ground-truth. In fact, the candidate $v s$ ground-truth Dice coefficient is $0.22 \pm 0.14$, including missing nodules. Fig. 4.5a shows a representaive example of a reconstructed nodule.

Changing the weights between the different LoG filters improves the segmentation results because it allows to include more subtle and larger strucutres. However, it was found that the connection between different structures increases, affecting the FP reduction even more than the under-segmentation. The effects of this under-segmentation will be discussed in the next section.

Table 4.4: Maximum sensitivity values (\%) for juxta-pleural nodules reported by Retico et al. (2008); De Nunzio et al. (2011); Han et al. (2015)

\begin{tabular}{ccccc} 
& Retico & De Nunzio & Han & Our method \\
\hline Sensitivity & 94.4 & 92.3 & 100 & 92.1 \\
FPs/scan & 546 & - & - & 3450 \\
Number of nodules & 102 & 78 & 323 & 510 \\
\hline
\end{tabular}




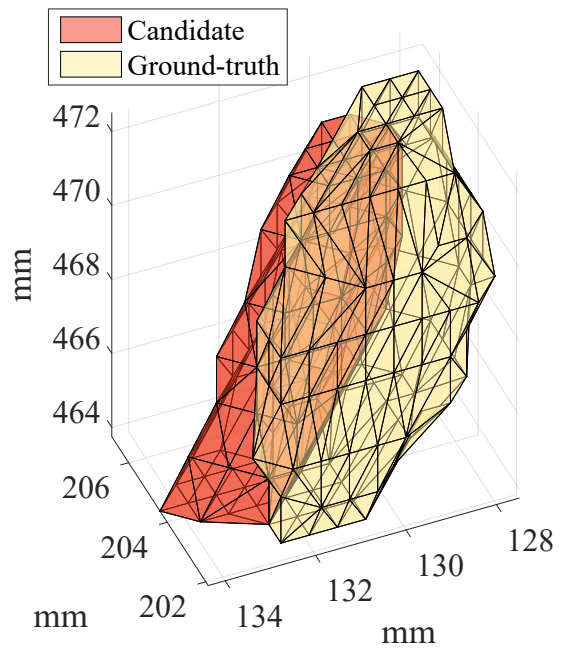

(a) 3D reconstruction.

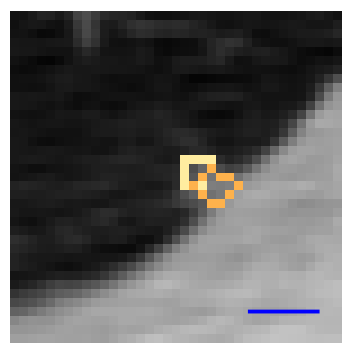

(b) 0520,371

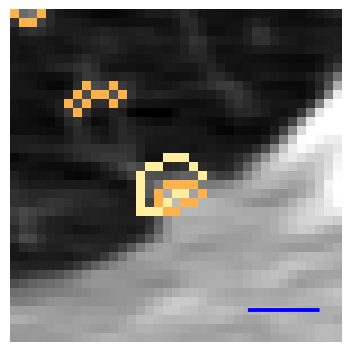

(d) 0520,376

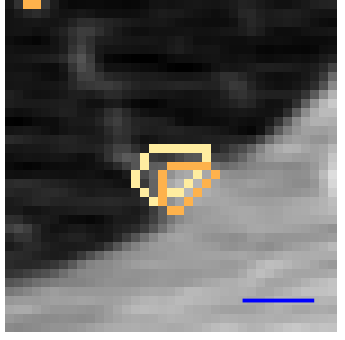

(c) 0520,374

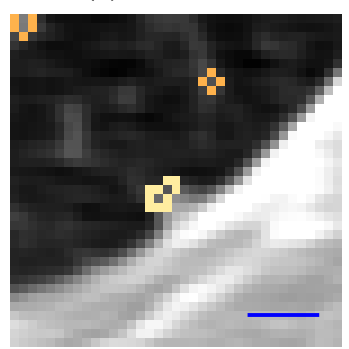

(e) 0520,378

Figure 4.5: 3D reconstruction of a juxta-pleural lung nodule candidate (orange) along with the ground-truth (yellow). The correspond initial, middle and final slices are shown. Each example is retrieved from a different slice (LIDC-IDRI case\#, slice\#). Blue scale bar corresponds to $5 \mathrm{~mm}$.

After the candidate detection step, any candidate with at least 1 voxel overlapping the groundtruth is considered a TP. Otherwise, it is considered as a FP. Nodules that correspond to more than one candidate are only considered once. The maximum sensitivity achieved by our system per nodule texture and agreement level among specialists is shown in Table 4.3 To achieve the results shown in Table 4.3, 3450 \pm 2272 FPs are detected per scan. Our detection system is able to identify the majority of the solid and sub-solid juxta-pleural nodules with radius $\leq 5 \mathrm{~mm}$. Fig. $4.6 \mathrm{a}$, Fig. 4.6b and Fig. 4.6c show examples of solid nodules, well contrasted with the non-parenchymal tissue, being properly detected. Fig. 4.6d shows a detected sub-solid nodule. Note that only the periphery of the nodule is not detected due to the lack of contrast.

The overall sensitivity of the proposed juxta-pleural nodule detection method is $92.1 \%$. Considering only solid and sub-solid nodule textures, the sensitivity increases to $95.3 \%$, which is near the maximum sensitivity limit referred. The performance of the system further improves if an agreement level $\geq 2$ is considered. On the other hand, the detection sensitivity for the non-solid nodules is poor, with only approximately half of these nodules being detected. Non-solid nodules, especially small ones, are quite challenging to detect due to the lack of contrast. For instance, the nodule shown in Fig. 4.6e is not detected. A different enhancement technique, such as a texturerelated approach, should be employed to detect these abnormalities.

The maximum sensitivities achieved by Retico et al. (2008); De Nunzio et al. (2011); Han et al. (2015) are shown in Table 4.4. Retico et al. (2008) considered only small solid nodules whereas Han et al. (2015) considered all the LIDC-IDRI radii range. De Nunzio et al. (2011) does not state either the nodule radius or nodule texture of their dataset. However, as their method is based on the localization of deformations caused by lung nodules, it is assumable that the searched 


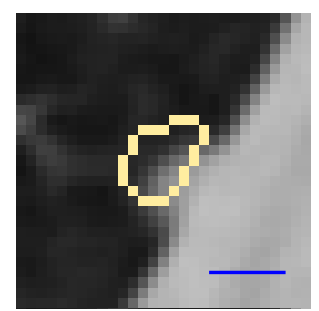

(a) 0005,89

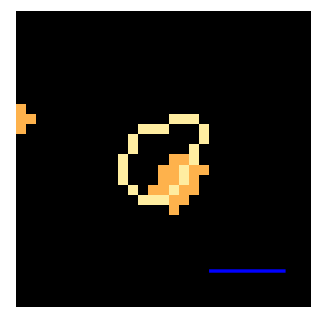

(f) 0005,50

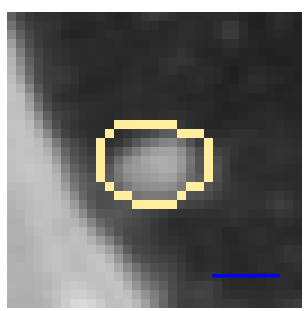

(b) 0040,44

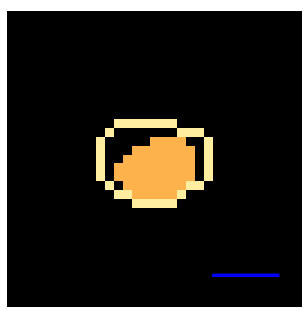

(g) 0040, 44

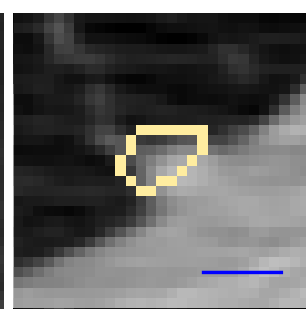

(c) 0520,374

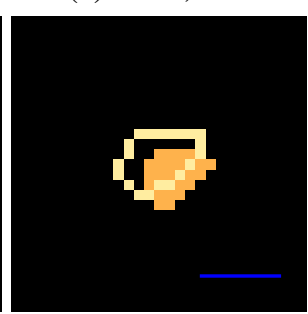

(h) 0520,374

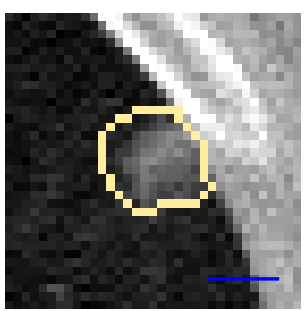

(d) 0312,118

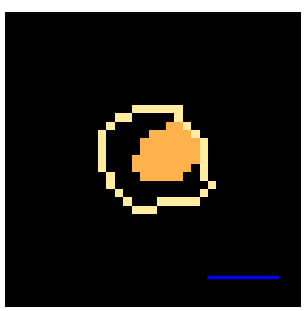

(i) 0312,118

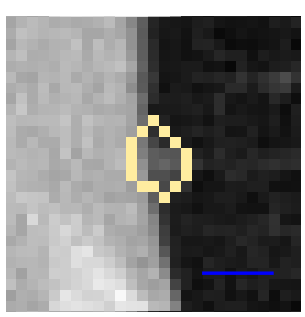

(e) 0721,85

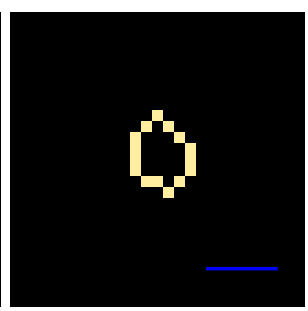

(j) 0721,85

Figure 4.6: Juxta-pleural lung nodules with radius $\leq 5 \mathrm{~mm}$ (first row) and respective detection (second row). Each example is retrieved from a scan and slice (LIDC-IDRI case\#, slice\#). Blue scale bar corresponds to $5 \mathrm{~mm}$.

nodules are solid. Their evaluation is not performed using the LIDC-IDRI and so the difficulty of the images and number of FPs produced are not directly comparable. Retico et al. (2008) achieved a maximum sensitivity of $94.4 \%$ and De Nunzio et al. (2011) of $92.3 \%$, whereas our system achieves $95.3 \%$ for sub-solid and solid nodules. Both Retico et al. (2008) and De Nunzio et al. (2011) search concavities in the lung volume segmentation to detect candidates. Consequently, their method fails if no deformation occurs. For instance, some juxta-pleural nodules are connected to the pleura with a subtle peduncle (Fig. 4.3b) and thus do not cause deformations of the lung segmentation border. In fact, De Nunzio et al. (2011) states that all the missed nodules are connected with peduncles. Our method, on the other hand, searches candidates inside the rectified lung volume. Consequently, the risk of failing the detection of juxta-pleural nodules that do not cause deformations on the mask is reduced.

Han et al. (2015) achieves $100 \%$ sensitivity. This work only considers solid and sub-solid nodules, which increases the system's performance. Furthermore, the lower number of scans might have allowed to avoid some of the most difficult juxta-pleural nodules. Note that, similarly to our work, the authors also perform morphological operations to rectify the lung volume and thus their system could miss the same nodules as ours. Statistically our results are more robust because a higher number of nodules and scans are used.

The maximum sensitivity of our candidate detection method is satisfactory when considering the difficult location of the juxta-pleural nodules. Our method studies a large number of nodules and scans in comparison with other works. Despite the good performance, specifically for subsolid and solid nodules, a more refined lung mask is needed to include the solid nodules that appear near the terminal regions of the lungs. Also, there is need to use a to better method to enhance sub-solid and specially non-solid nodules in order to improve our results. 
Table 4.5: Maximum achieved sensitivity values (\%) for the proposed method, after the false positive reduction step, per nodule texture and minimum agreement level between specialists.

\begin{tabular}{ccccc} 
& \multicolumn{4}{c}{ Nodule texture } \\
\cline { 2 - 5 } Agreement level & Non-solid & Sub-solid & Solid & Overall \\
\hline At least 4 & 20.0 & 80.0 & 87.8 & 84.3 \\
At least 3 & 14.3 & 88.2 & 87.6 & 85.2 \\
At least 2 & 13.3 & 69.0 & 85.9 & 80.9 \\
At least 1 & 25.0 & 60.5 & 78.3 & 72.5 \\
\hline Number of nodules & 42 & 45 & 423 & 510 \\
\hline
\end{tabular}

\subsection{False positive reduction}

The proposed cadidate detection step produces an average of $3450 \pm 2272 \mathrm{FPs} / \mathrm{scan}$. This number of FPs is significantly reduced by the fixed-rules based reduction and the supervised FP reduction. The performance is mostly affected by the fixed-rules based reduction, as discussed in this section.

\subsubsection{Fixed-rules based FP reduction}

The most obvious non-nodule candidates are eliminated via fixed-rules. First, ensuring a safety margin due to the possibility of small attached structures, all candidates with radius $\geq 6 \mathrm{~mm}$ along with those that dist more than $6 \mathrm{~mm}$ from the pleural wall are eliminated. After this step, the number of FPs/scan is reduced to $352 \pm 154$ and our maximum sensitivity also drops to approximately $86 \%$. The wrongly-eliminated nodules usually correspond to abnormalities that are connected to blood vessels or noisy structures, as exemplified in Fig. 4.7a Juxta-vascular nodules also require attention from a dedicated system and thus their detection by our system should not be prioritized. Then, the filtering using the radius $\leq 1.5 \mathrm{~mm}$ criteria reduces our sensitivity to $72.5 \%$ with $95.5 \pm$ 52.1 FPs/scan. The maximum sensitivity achieved after this step is shown in Table 4.5 .

The removal of candidates with radius $\leq 1.5 \mathrm{~mm}$ is a critical step of our system. Without this step the high number of FPs would not allow to achieve a good classification performance by either of the metrics referred in Section 3.6. On the other hand, our maximum sensitivity highly penalized by this reduction. The drop in the sensitivity value results from the poor segmentation of the nodules. Recall that average Dice coefficient prior to FP reduction is just $0.22 \pm 0.14$. Consequently, for the smaller nodules the segmentation is expected to be bellow the defined threshold, affecting the final sensitivity. In fact, sub-solid and non-solid nodules are the most affected by the fixed rules-based reduction, as visible in Table 4.5 and illustrated by Fig. 4.7b and Fig. 4.7c

The initial FP reduction step is not avoidable due to the large number of FPs that result from small blood vessels along the lung parenchyma. Some characteristic examples of non-nodule candidates are shown in Fig. 4.7d and Fig. 4.7e. Furthermore, although our sensitivity reduction is much higher than Han et al. (2015) (97\% with approx. $60 \mathrm{FPs} / \mathrm{scan})$, our fixed-rules are only nodule-dependent and do not rely on prior knowledge of the candidates properties. Despite the low overall sensitivity, our system is capable of retaining almost $80 \%$ of the solid nodules. 


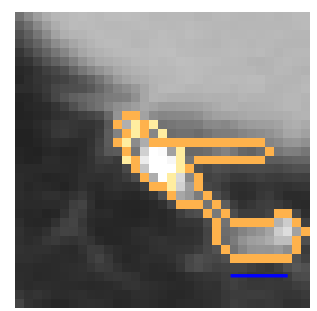

(a) 0063,61

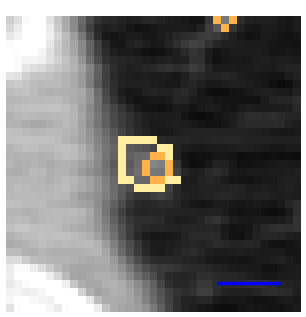

(b) 0677,374

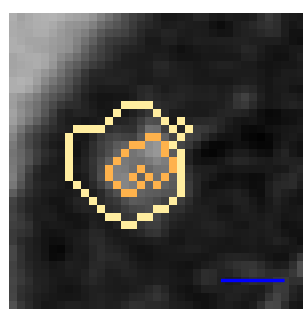

(c) 0743,17

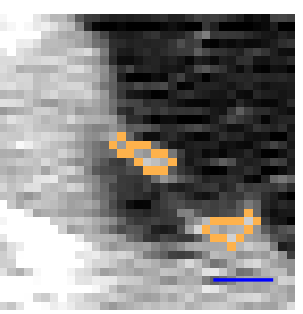

(d) 0820,215

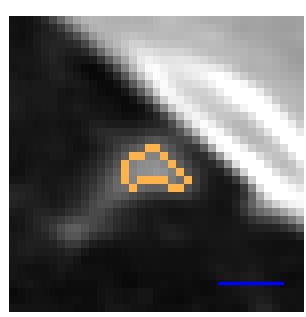

(e) 0634,132

Figure 4.7: Juxta-pleural lung nodule candidates marked with orange contour. The yellow contour corresponds to the ground-truth. Each example is retrieved from a scan and slice (LIDC-IDRI case\#, slice\#). Blue scale bar corresponds to $5 \mathrm{~mm}$.

Generically, there is the need to improve the nodule segmentation after the initial candidate detection. Improving the segmentation will allow to retain a larger number of nodules after the reduction with the fixed rules. A possible solution is to use a 3D active contour initialized from a sphere with the candidate's diameter, as suggested by Cascio et al. (2012), because it allows to constraint the segmentation and thus avoid the risk of leaking of region-growing based methods. Segmenting the vessel tree is also of interest, as some candidates occur in blood vessels that show as blob structures on certain slices. Furthermore, there is also the possibility to replace the fixed rules by a first and simpler supervised learning approach as performed by Murphy et al. (2009).

\subsubsection{Supervised learning FP reduction}

The candidates that remain after the fixed-rules based reductions are equally divided, scan-wise, in test and training sets. The SMV-rbf classifier is trained using the training set and tested in the rest of the candidates. This process is repeated $100 \times$ and the average ROC curve $( \pm$ standard deviation) is obtained. Fig. $4.8 \mathrm{a}$ shows the ROC curve of our classifier.

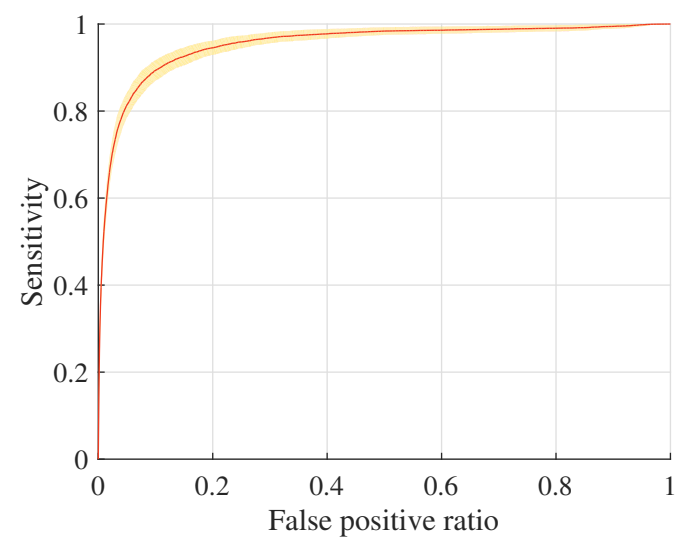

(a) Classifier ROC curve.

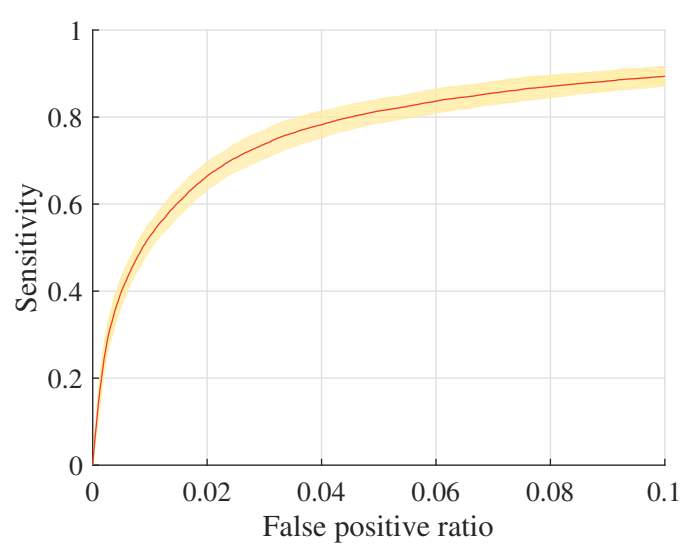

(b) Classifier ROC curve detail.

Figure 4.8: ROC curves of the proposed classifier for the detection of juxta-pleural nodules with radius $\leq 5 \mathrm{~mm}$ after the false positive reduction. The standard deviation is shown in yellow. Specialists' agreement level is at least 1. 
Table 4.6: Area under the ROC curve of the classifiers used to reduce the number of juxta-pleural nodules reported by Retico et al. (2008); De Nunzio et al. (2011); Han et al. (2015).

\begin{tabular}{ccccc} 
& Retico & De Nunzio & Han & Our method \\
\hline AUC & $0.88 \pm 0.07$ & 0.84 & $0.97 \pm 0.01$ & $0.95 \pm 0.01$ \\
\hline
\end{tabular}

For our method it is expected that the number of FPs is reduced by at least $10 \times$ in order to achieve results comparable with the literature. Consequently, a fast evolution of the ROC curve for the first 0.1 FP ratio is essential. From the observation of Fig. $4.8 \mathrm{~b}$ it is visible that reducing the number of FPs to $1 / 10$ our sensitivity drops approximately $10 \%$. It is thus noticeable that the fixed-rules based reduction is the limiting performance step of our method.

The fast evolution of the curve for low false positive ratios is related to the area under the ROC curve (AUC). Consequently, the performance of the supervised learning FP reduction step can be evaluated in terms of AUC. Table 4.6 shows the AUC obtained for the works currently being discussed. De Nunzio et al. (2011) used features related to intensity and geometry. Retico et al. (2008) also used hessian-related features. Han et al. (2015) used gradient and intensity features, achieving the highest AUC of the reviewed works. Gradient features appear to be important to the performance of the classification, being used by the works with highest AUC. Han et al. (2015) also reported a performance drop when considering geometric features. This is most likely due to the fact the their method already considers multiple geometry features for rule-based FP reduction.

The performance of our classifier is similar to Han et al. (2015), even though our candidate segmentation is most likely poorer and non-solid nodules are also considered. Feature selection to remove redundant features should further improve our results. Nevertheless, it is proven that the limiting step in our algorithm is the fixed-rules based FP reduction.

\subsection{Comparison with existing methods}

The overall performance of the proposed method in terms of FROC curve is shown in Fig. 4.9 , Note that our maximum sensitivity is $72.5 \%$, namely due to the poor FP reduction resultd using fixed-rules. With $4 \mathrm{FPs} / \mathrm{scan}$ our system achieves a sensitivity of $57.2 \%$ and $63.5 \%$ with 8 FPs/scan. For solid nodules, which correspond to the majority of the studied cases, our method achieves sensitivities of $61.8 \%$ and $68.5 \%$, respectively. Our average score (Section 3.6) is computed by averaging the values highlighted in Fig. 4.9b. Table 4.7 shows the final performance of our system in terms of sensitivity and score for the different nodule textures.

Table 4.7: Performance of the proposed juxta-pleural lung nodule detection method for different nodule textures.

\begin{tabular}{lccc|c} 
Nodule texture & Nodules & FPs/scan & Sensitivity $(\%)$ & Score \\
\hline All textures & 510 & 4 & 57.2 & 0.39 \\
Sub-solid and solid & 466 & 4 & 60.5 & 0.41 \\
Solid & 423 & 4 & 61.8 & 0.42
\end{tabular}


Table 4.8: Juxta-pleural lung nodule detection performance of different systems. $r$ is the nodule radius. A - Retico et al. (2008), B - De Nunzio et al. (2011), C - Han et al. (2015), D - Fujitalab, E - Region growing volume plateau, F - Channeler Ant model, G - Voxel-based neural approach, H - ISI-CAD, I - Philips Lung Nodule CAD (van Ginneken et al. (2010)).

\begin{tabular}{lcccccc|c} 
System & Dataset & Scans & Nodules & $r(\mathbf{m m})$ & FPs/scan & Sensitivity (\%) & Score \\
\hline A & Private & 42 & 25 & $\geq 2.5$ & 6 & 72.0 & - \\
B & Private & - & - & - & - & 66.5 & - \\
C & LIDC-IDRI & $\mathbf{2 0 5}$ & $\mathbf{3 2 3}$ & $\geq 1.5$ & $\mathbf{4 . 1}$ & $\mathbf{8 9 . 2}$ & - \\
D & ANODE09 & 50 & 60 & $\geq 4$ & 4 & 15.3 & 0.10 \\
E & ANODE09 & 50 & 60 & $\geq 4$ & 4 & 33.9 & 0.16 \\
F & ANODE09 & 50 & 60 & $\geq 4$ & 4 & 35.6 & 0.21 \\
G & ANODE09 & 50 & 60 & $\geq 4$ & 4 & 35.6 & 0.19 \\
H & ANODE09 & $\mathbf{5 0}$ & $\mathbf{6 0}$ & $\geq 4$ & $\mathbf{4}$ & $\mathbf{6 9 . 5}$ & $\mathbf{0 . 4 4}$ \\
I & ANODE09 & 50 & 60 & $\geq 4$ & 4 & 22.9 & 0.14 \\
\hline Ours & LIDC-IDRI & $\mathbf{3 1 5}$ & $\mathbf{5 1 0}$ & {$[1.5-5]$} & $\mathbf{4}$ & $\mathbf{5 7 . 4}$ & $\mathbf{0 . 3 9}$ \\
\hline
\end{tabular}

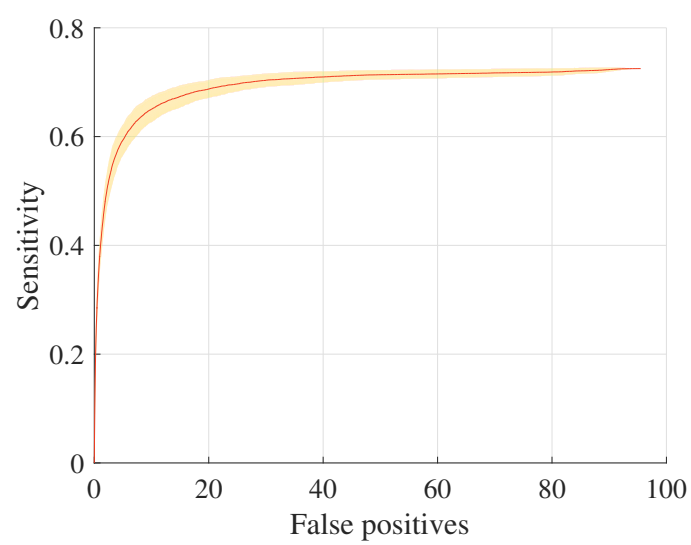

(a) FROC curve.

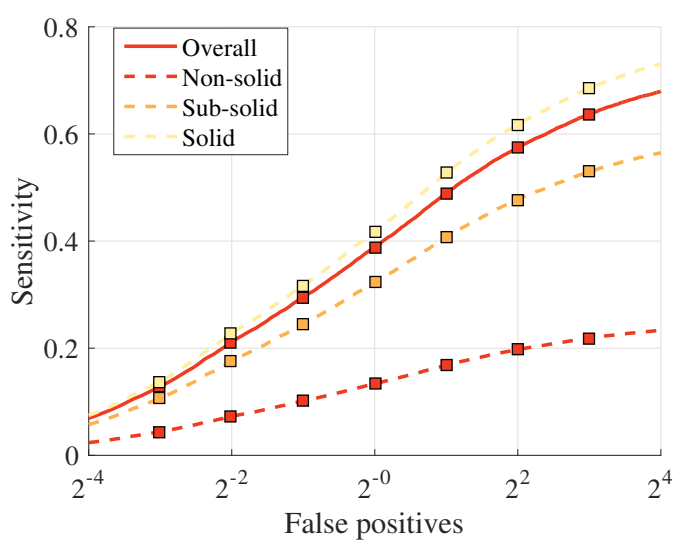

(b) FROC curve detail.

Figure 4.9: FROC curves of the proposed system for the detection of juxta-pleural nodules with radius $\leq 5 \mathrm{~mm}$ after the false positive reduction. Specialists' agreement level is at least 1 .

Table 4.8 shows a comparison of our results with those of Retico et al. (2008); De Nunzio et al. (2011); Han et al. (2015) as well as the works compared in van Ginneken et al. (2010) in terms of FPs/scan and average score. The majority of the nodules evaluated in these works has solid texture. As a note, please consider that the comparison between works that do not use the same set of nodules is difficult due to the differences between both ground-truth and images.

Sensitivity-wise, Han et al. (2015) have the best performance, achieving 89\% with $4 \mathrm{FPs} / \mathrm{scan}$. The used approach, vector quantization, is interesting for juxta-pleural detection as it exploits the intensity differences between structures. However, other methods such as the Region growing volume plateau and the Channeler Ant model also use intensity based approaches to detect their candidates. Despite that, their performance is significantly worse, with sensitivities bellow $50 \%$. The most likely reason for this is that these methods do not properly explore the boundary regions of the lung volume as they are region-growing based. Also, their FP reduction is not as effective. 
On the other hand, methods specifically designed to detect juxta-pleural nodules, Retico et al. (2008); De Nunzio et al. (2011) and Voxel-based neural approach do not out-perform intensitybased methods, even though they are dedicated. These approaches try to detect the deformations caused by the nodules on the lung mask. However, this method assumes that all juxta-pleural nodules will cause deformations, both 2D and 3D, that they are not attached by peduncles and that the natural noise created by the shape of the lungs' boundaries between slices is irrelevant. If these conditions are not met the methods tend to under-perform, explaining the obtained results. Finally, methods such as ISI-CAD search candidates by their shape characteristics. The performance of this method is affected by juxta-pleural nodules because they are not as rounded as isolated nodules. Despite that, both sensitivity and score are good in comparison to other methods, having achieved an accuracy and a score of $69.5 \%$ and 0.44 , respectively.

The proposed method is designed to combine intensity-based and shape-based approaches. Consequently, the structure of our system is closer to those of Han et al. (2015) and ISI-CAD. We avoid searching for deformations of the lungs' limits and instead search inside a rectified lung volume segmentation, which proved to be advantageous because we can also find nodules connected with peduncles, as well as sub-solid and non-solid nodules.

Our score and sensitivity are similar to the ones achieved by ISI-CAD when considering solid nodules. When comparing to Han et al. (2015), however, our performance is significantly worse. Our performance difference with these two best works can be partially explained by the differences between the two works. Han et al. (2015) did not do a proper selection of the nodules considered as juxta-pleural, as discussed before, which may be inadvertently improving their results. Furthermore, the evaluation of these two methods considered less scans and less nodules than ours, and consequently our results might have been hindered by more complicated cases. Furthermore, our method also focuses on a smaller average nodule radii, increasing the difficulty of the detection process (Setio et al. (2015)). Finally, our initial candidate reduction is worse than those of these methods. Han et al. (2015) use an adapted set of fixed-rules that significantly reduces the number of FPs without compromising the sensitivity. However, the rules are biased by the data in question. The ISI-CAD method uses k-NN classification to perform an initial candidate reduction. This is pertinent as it is less data-dependent than the fixed rules. However, using two consecutive classifiers would be impracticable in our method due to the high initial number of FP. A possible solution would be to replace the fixed-rules step by one that uses fixed-rules to remove the larger nodules and then a simple classifier to distinguish between small nodule and non-nodule structures. The remaining FP would then be analyzed by the proposed SVM-rbf classifier. 


\subsection{Concluding remarks}

The evaluation of the proposed method to detect juxta-pleural lung nodules in CT scans is presented in this section. We discuss the improvement of the lung volume segmentation, the performance of the candidate detection as well as the FP reduction. An overall comparison with state-of-the-art methods that refer their performance for juxta-pleural nodules is also presented.

A characterization of the juxta-pleural lung nodule dataset is performed. In comparison to the nodules available in the LIDC-IDRI, juxta-pleural nodules present a lower agreement level among the specialists and are more subtle. These characteristics, along with the inherent location of the nodules, lead to an increase in the detection difficulty.

The proposed refinement of the lung volume segmentation has proven to improve the performance of the system, both in terms of number and volume of the nodules included in the segmentation. The majority of the nodules not included corresponds to solid nodules that occur near the upper and lower limits of the lungs. A possible solution to include these nodules is to use an average 3D model of the lungs and perform the active contour refinement 3D-wise.

Our candidate detection method shows high sensitivity values for solid and sub-solid nodules. On the other hand, the lack of contrast of non-solid nodules hinders their detection. Furthermore, the overall segmentation of the nodules is poor. Improvements in the segmentation could be performed by changing the weight of the different LoG filters. However, this increases the risk of attachment between structures. The under-segmentation does not affect the detection sensitivity but affects the reduction of FPs.

The reduction of the number of FPs is divided in two steps. In the proposed method the step that most affects the system's performance is the FP reduction via fixed rules. Due to the the under-segmentation, the filtering of low-radii candidates significantly reduces our sensitivity. Consequently, a proper candidate segmentation has to be performed in order to improve the results. Due to the risk of leakage of region-growing approaches, 3D segmentation using active contours is most-likely a solution for this problem. The second step, which uses supervised learning, shows a performance similar to the state-of-the-art works. Further improvement of the classifier can be performed with feature selection to remove highly correlated features.

Our system shows a performance similar or better than other state-of-the-art works. In fact, the proposed method combines the basic idea of the two best performing algorithms, exploiting both the difference intensity between solid nodules and parenchymal tissue and the natural roundness of the nodules. The studied dataset can be considered of increased difficulty due to the small nodule radius and thus our method presents itself as a good starting point for a high performance system dedicated to the detection of juxta-pleural lung nodules. The implementation of the improvements referred previously is expected to further increase the overall performance of our system. 


\section{Chapter 5}

\section{Conclusion and Future work}

Second-opinion CADe systems are useful to increase the overall cancer detection performance of physicians. Several approaches regarding lung nodule detection in CT scans have already been proposed and showed good detection success. However, the performance of these systems drops for juxta-pleural nodules, which are abnormalities that occur near the pleural wall. Despite that, there are few works addressing specifically this type of nodule.

A juxta-pleural lung nodule detection method is proposed. For that purpose, a juxta-pleural lung nodule sub-dataset from the LIDC/IDRI dataset was created. This sub-dataset considers the majority of the LIDC/IDRI scans and was manually revised to only include juxta-pleural nodules.

Our method exploits the intensity difference between nodules and parenchymal tissue and the natural roundness of these structures. First, a lung volume segmentation properly rectified with an active contour-based approach to include juxta-pleural nodules is performed. This rectification improves the inclusion of juxta-pleural nodules of the C-BER group lung volume segmentation algorithm. Then, candidates are detected by selecting an appropriate threshold on a sliding window. The more subtle candidates are enhanced with Laplacian-of-Gaussian filtering. Then, FPs are reduced via fixed-rules and supervised learning.

The juxta-pleural lung nodule detection method achieves high sensitivity values prior to the FP reduction with fixed-rules and supervised learning. For this first step, missed nodules are essentially non-solid nodules, which detection is not trivial due to the lack of contrast. However, some solid nodules near the limits of the lungs are also not detected. There is thus need to improve the initial lung volume segmentation to include these most challenging nodules. Our overall sensitivity significantly drops after the fixed-rules based reduction. This happens essentially due to a poor segmentation of the candidates. It is demanding that a proper FP reduction is performed. There are numerous candidates occurring on blood vessels and other blob-like structures that must be removed from the candidate list prior to the supervised learning FP reduction. Due to the under-segmentation, these candidates end up having the same characteristics as the detected nodules. Consequently, their elimination also results in the elimination of TP candidates, affecting our results. With this in mind, the following future improvements are suggested: 
1. Lung volume segmentation: there is the need to improve the lung volume segmentation so that solid nodules that occur near the limits of the lungs are also detected. A possible approach is to use $3 \mathrm{D}$ active contour with an average $3 \mathrm{D}$ model of the lung mask.

2. FP reduction: a proper FP reduction is essential to obtain an algorithm with excellent performance. The proposed FP reduction schemes fails due to a poor 3D candidate segmentation and selection of blood vessels as candidates. Consequently, it is of interest to:

- Improve the candidate 3D segmentation. Due to the position of juxta-pleural nodules, region-growing based approaches are not recommended. A possible solution is to use $3 \mathrm{D}$ active contour considering as the initilization model a sphere with the same diameter of the candidate.

- Segment the blood vessel tree using, for instance, a region-growing based approach. This segmentation needs to be precise so that nodules are not included.

Despite the detected problems, our dedicated CADe has a performance similar to state-of-theart works and consequently shows great potential for the detection of juxta-pleural lung nodules. The implementation of the previously referred suggestions, and consequent performance improvement, will further increase the importance of the integration of the proposed method on a complete, multi-dedicated CAD system. 


\section{Appendix A}

\section{Juxta-pleural lung nodules}

The list of the juxta-pleural lung nodules available in the LIDC-IDRI dataset is presented in the next pages. The number of the LIDC-IDRI case, the first and last slice of nodule and the its radius are shown. The information is divided in 6 different pages for convenience. 
Table A.1: Juxta-pleural lung nodules available in the LIDC-IDRI dataset (1 out of 6)

\begin{tabular}{|c|c|c|c|c|c|c|c|c|}
\hline LIDC-IDRI & Slice range & Radius (mm) & LIDC-IDRI & Slice range & Radius (mm) & LIDC-IDRI & Slice range & Radius (mm) \\
\hline-1 & $87-95$ & 13,08 & -46 & $20-22$ & 3,51 & -118 & $66-69$ & 3,4 \\
\hline-2 & $172-199$ & 13,21 & & $33-36$ & 4,52 & & $69-72$ & 3,27 \\
\hline \multirow[t]{2}{*}{-3} & $82-89$ & 7,72 & & $38-39$ & 3,3 & -121 & $156-167$ & 8,69 \\
\hline & $82-85$ & 5,73 & & $39-45$ & 4,87 & -124 & $64-66$ & 3,14 \\
\hline-5 & $88-89$ & 3,03 & -53 & $33-41$ & 12,39 & -125 & $110-113$ & 3,38 \\
\hline-7 & $119-130$ & 8,53 & & $46-49$ & 6,2 & -126 & $88-90$ & 3,65 \\
\hline-9 & $103-105$ & 2,91 & -55 & $123-125$ & 5,44 & -128 & $171-178$ & 7,04 \\
\hline \multirow[t]{2}{*}{-10} & $68-71$ & 2,66 & -57 & $154-177$ & 11,97 & -129 & $61-63$ & 2,3 \\
\hline & $93-97$ & 3,67 & -58 & $87-90$ & 4,94 & & $112-115$ & 3,21 \\
\hline \multirow[t]{5}{*}{-11} & $28-32$ & 5,19 & & $104-111$ & 11,18 & & $117-122$ & 4,79 \\
\hline & $48-49$ & 2,47 & -59 & $100-103$ & 6,21 & -131 & $119-123$ & 2,61 \\
\hline & $58-60$ & 3,3 & -60 & $91-100$ & 10,46 & -132 & $13-14$ & 2,89 \\
\hline & $94-97$ & 3,46 & -61 & $37-42$ & 10,32 & & $23-31$ & 11,3 \\
\hline & $104-106$ & 3,14 & & $48-70$ & 16,75 & -133 & $36-44$ & 7,06 \\
\hline \multirow[t]{4}{*}{-12} & $66-68$ & 3,49 & & $86-100$ & 17,37 & & $131-143$ & 8,1 \\
\hline & $70-73$ & 4,48 & & $88-91$ & 6,17 & -135 & $66-67$ & 3,2 \\
\hline & $77-80$ & 4,7 & & $90-92$ & 5,21 & -136 & $26-31$ & 7,85 \\
\hline & $79-81$ & 3,78 & -63 & $60-61$ & 2,96 & & $64-65$ & 3,26 \\
\hline-19 & $254-277$ & 12,78 & & $89-94$ & 6,73 & & $72-73$ & 4,11 \\
\hline-21 & $61-64$ & 5,01 & -67 & $111-120$ & 5,67 & & $77-77$ & 2,82 \\
\hline-22 & $101-111$ & 14,66 & & $173-175$ & 3,03 & -137 & $31-32$ & 2,81 \\
\hline-23 & $113-124$ & 13,85 & -68 & $118-122$ & 3,34 & & $99-109$ & 11,44 \\
\hline \multirow[t]{2}{*}{-24} & $53-54$ & 3,34 & & $154-160$ & 4,78 & -139 & $25-39$ & 14,14 \\
\hline & $71-75$ & 7,32 & & $167-169$ & 2,49 & & $28-37$ & 14,6 \\
\hline-26 & $90-91$ & 2,6 & & $204-212$ & 5,63 & -141 & $149-191$ & 17,6 \\
\hline \multirow[t]{3}{*}{-27} & $83-84$ & 3,42 & & $211-220$ & 6,64 & & $207-212$ & 3,21 \\
\hline & $83-85$ & 4,5 & -70 & $70-71$ & 2,58 & & $316-320$ & 3,69 \\
\hline & $112-113$ & 4,36 & -73 & $56-58$ & 5,59 & -142 & $127-135$ & 6,59 \\
\hline-30 & $50-52$ & 4,11 & & $108-113$ & 9,05 & & $231-238$ & 4,87 \\
\hline-31 & $57-57$ & 2,77 & -75 & $86-88$ & 2,48 & -148 & $63-63$ & 2,22 \\
\hline-33 & $108-113$ & 5,98 & & $217-224$ & 5,94 & -149 & $53-56$ & 4,56 \\
\hline-34 & $97-100$ & 3,06 & -76 & $129-134$ & 4,09 & & $71-73$ & 3,67 \\
\hline-37 & $80-86$ & 8,59 & -81 & $94-103$ & 10,82 & & $85-85$ & 2,14 \\
\hline \multirow[t]{2}{*}{-39} & $132-136$ & 3,5 & -82 & $167-181$ & 10,28 & & $98-99$ & 2,94 \\
\hline & $163-167$ & 4 & -83 & $51-53$ & 3,27 & -158 & $51-57$ & 4,34 \\
\hline-40 & $43-45$ & 4,02 & & $114-118$ & 3,62 & & $128-133$ & 3,72 \\
\hline \multirow[t]{3}{*}{-41} & $46-54$ & 10,18 & -85 & $183-185$ & 2,77 & & $135-143$ & 4,21 \\
\hline & $56-57$ & 2,98 & -87 & $104-112$ & 11,63 & & $140-146$ & 5,08 \\
\hline & $124-126$ & 3,35 & -91 & $32-35$ & 2,82 & -160 & $64-68$ & 5,6 \\
\hline-42 & $78-80$ & 3,85 & & $57-63$ & 4,83 & & $72-74$ & 3,73 \\
\hline \multirow[t]{2}{*}{-43} & $83-89$ & 8,33 & -92 & $211-214$ & 2,87 & -161 & $37-43$ & 4,95 \\
\hline & $89-95$ & 9,46 & -95 & $187-203$ & 12,92 & & $82-90$ & 5,23 \\
\hline \multirow[t]{7}{*}{-45} & $24-28$ & 4,76 & -98 & $28-32$ & 6,19 & & $83-86$ & 3,26 \\
\hline & $41-42$ & 3,8 & -101 & $80-81$ & 3,79 & -162 & $75-76$ & 2,86 \\
\hline & $49-53$ & 6,43 & -102 & $43-44$ & 3,86 & -163 & $36-45$ & 11,57 \\
\hline & $62-64$ & 4,16 & -108 & $115-118$ & 4,24 & -164 & $158-162$ & 3,82 \\
\hline & $68-72$ & 5,04 & -109 & $109-117$ & 6,42 & & $215-227$ & 6,32 \\
\hline & $81-87$ & 8,31 & -111 & $93-96$ & 5,65 & -165 & $121-122$ & 3,97 \\
\hline & $86-89$ & 5,43 & -114 & $86-93$ & 6,41 & -166 & $50-64$ & 16,17 \\
\hline
\end{tabular}


Table A.2: Juxta-pleural lung nodules available in the LIDC-IDRI dataset (2 out of 6)

\begin{tabular}{|c|c|c|c|c|c|c|c|c|}
\hline LIDC-IDRI & Slice range & Radius (mm) & LIDC-IDRI & Slice range & Radius (mm) & LIDC-IDRI & Slice range & Radius (mm) \\
\hline-167 & $51-52$ & 3,13 & & $109-116$ & 8,62 & -312 & $48-56$ & 4,95 \\
\hline \multirow[t]{2}{*}{-169} & $96-98$ & 2,81 & -232 & $90-91$ & 3,02 & & $52-55$ & 3,46 \\
\hline & $127-133$ & 3,93 & -235 & $75-78$ & 5,86 & & $71-76$ & 3,67 \\
\hline-170 & $108-121$ & 11,21 & -236 & $98-102$ & 5,47 & & $117-120$ & 3,41 \\
\hline \multirow[t]{2}{*}{-171} & $54-54$ & 2,44 & -237 & $69-71$ & 4,84 & -315 & $16-18$ & 4,32 \\
\hline & $72-72$ & 2,88 & -240 & $60-66$ & 10,24 & & $36-39$ & 5,12 \\
\hline-173 & $76-78$ & 3,61 & -241 & $108-111$ & 3,46 & & $38-41$ & 5 \\
\hline-176 & $172-173$ & 2,79 & & $113-115$ & 3,02 & & $47-50$ & 6,85 \\
\hline-178 & $77-78$ & 2,82 & -243 & $56-58$ & 6,4 & & $50-51$ & 2,83 \\
\hline-179 & $87-90$ & 4,45 & -245 & $60-61$ & 3,19 & & $80-84$ & 4,68 \\
\hline-181 & $90-92$ & 3,08 & -246 & $94-96$ & 5,2 & -318 & $226-236$ & 4,61 \\
\hline \multirow[t]{2}{*}{-185} & $33-37$ & 5,29 & -249 & $28-34$ & 6,16 & -319 & $152-157$ & 4,55 \\
\hline & $65-66$ & 3,52 & & $80-96$ & 8,82 & -320 & $134-137$ & 3,58 \\
\hline \multirow[t]{2}{*}{-186} & $112-120$ & 13,29 & -250 & $89-91$ & 2,89 & -321 & $131-135$ & 3,76 \\
\hline & $118-123$ & 8,93 & -254 & $40-41$ & 2,87 & -323 & $40-41$ & 3,42 \\
\hline-187 & $119-126$ & 11,94 & -257 & $67-72$ & 7,31 & & $44-46$ & 3,62 \\
\hline-188 & $52-65$ & 11,88 & -258 & $92-94$ & 4,68 & -328 & $290-293$ & 2,63 \\
\hline \multirow[t]{2}{*}{-190} & $46-56$ & 12,16 & -259 & $30-35$ & 6,54 & -332 & $249-251$ & 3,33 \\
\hline & $89-95$ & 9,37 & -260 & $56-59$ & 6,62 & -337 & $58-64$ & 8,6 \\
\hline-191 & $85-95$ & 14,79 & & $119-121$ & 3,09 & -339 & $232-233$ & 2,05 \\
\hline \multirow[t]{4}{*}{-192} & $76-78$ & 2,44 & -264 & $70-77$ & 4,95 & -340 & $32-88$ & 15,8 \\
\hline & $116-121$ & 5,32 & -265 & $100-115$ & 12,99 & -341 & $74-77$ & 2,98 \\
\hline & $143-146$ & 3,94 & -267 & $33-58$ & 17,38 & & $104-105$ & 2,56 \\
\hline & $164-167$ & 3,42 & & $132-134$ & 3,74 & & $108-109$ & 1,99 \\
\hline \multirow[t]{4}{*}{-195} & $38-41$ & 5,46 & -273 & $48-48$ & 2,35 & & $133-136$ & 3,36 \\
\hline & $42-47$ & 7,23 & -278 & $132-135$ & 3,06 & -344 & $168-173$ & 2,65 \\
\hline & $52-59$ & 9,8 & -280 & $194-196$ & 2,5 & & $292-294$ & 1,88 \\
\hline & $84-91$ & 8,14 & -281 & $50-52$ & 4,52 & & $295-313$ & 6,65 \\
\hline-198 & $30-32$ & 3,54 & -282 & $58-60$ & 4,08 & & $358-365$ & 3,55 \\
\hline-199 & $148-149$ & 2,16 & -284 & $111-113$ & 4,17 & -345 & $127-131$ & 3,26 \\
\hline \multirow[t]{2}{*}{-200} & $46-47$ & 3,27 & -285 & $80-83$ & 5,24 & -347 & $35-41$ & 11,85 \\
\hline & $60-62$ & 3,18 & -288 & $75-76$ & 2,55 & -350 & $186-189$ & 3,3 \\
\hline \multirow[t]{3}{*}{-201} & $38-40$ & 2,96 & -289 & $52-60$ & 14,06 & & $241-242$ & 2,13 \\
\hline & $58-68$ & 10,78 & & $60-60$ & 2,29 & -355 & $59-68$ & 11,15 \\
\hline & $110-111$ & 3,28 & -291 & $81-85$ & 3,62 & -356 & $116-119$ & 4,43 \\
\hline-202 & $41-42$ & 3,76 & -297 & $348-354$ & 3,13 & & $118-120$ & 3,44 \\
\hline \multirow[t]{2}{*}{-203} & $41-43$ & 4,38 & -298 & $75-82$ & 4,6 & & $128-129$ & 2,63 \\
\hline & $110-124$ & 14,49 & -299 & $365-373$ & 4,51 & -358 & $234-239$ & 3,47 \\
\hline \multirow[t]{2}{*}{-207} & $66-70$ & 7,86 & & $389-395$ & 3,92 & & $236-239$ & 2,82 \\
\hline & $175-177$ & 3,03 & & $456-462$ & 3,48 & & $250-253$ & 3,11 \\
\hline \multirow[t]{2}{*}{-211} & $68-88$ & 11,4 & -300 & $197-200$ & 2,65 & & $260-264$ & 3,24 \\
\hline & $114-117$ & 3,14 & -302 & $280-284$ & 2,95 & -362 & $36-42$ & 7,59 \\
\hline-213 & $90-94$ & 5,4 & -303 & $62-72$ & 15,41 & -369 & $226-229$ & 3,72 \\
\hline-215 & $146-148$ & 3,23 & -305 & $131-135$ & 5,46 & -370 & $158-168$ & 3,99 \\
\hline \multirow[t]{3}{*}{-220} & $135-143$ & 8,34 & & $191-198$ & 3,72 & -371 & $112-120$ & 4,14 \\
\hline & $141-147$ & 6,2 & -308 & $413-432$ & 6,5 & -374 & $116-118$ & 2,64 \\
\hline & $155-169$ & 8,75 & -309 & $20-24$ & 4,19 & -377 & $183-187$ & 4,26 \\
\hline \multirow[t]{2}{*}{-229} & $93-100$ & 10,05 & -311 & $144-180$ & 13,15 & -381 & $22-24$ & 3,16 \\
\hline & $101-104$ & 6,46 & & $189-196$ & 5,92 & -384 & $321-323$ & 3,11 \\
\hline
\end{tabular}


Table A.3: Juxta-pleural lung nodules available in the LIDC-IDRI dataset (3 out of 6)

\begin{tabular}{|c|c|c|c|c|c|c|c|c|}
\hline LIDC-IDRI & Slice range & Radius (mm) & LIDC-IDRI & Slice range & Radius (mm) & LIDC-IDRI & Slice range & Radius (mm) \\
\hline & $445-448$ & 2,98 & & $56-57$ & 3,71 & & $130-134$ & 1,92 \\
\hline \multirow[t]{2}{*}{-385} & $334-351$ & 6,5 & & $83-83$ & 2,79 & & $134-136$ & 2,46 \\
\hline & $346-378$ & 10,51 & -445 & $58-62$ & 3,54 & & $177-180$ & 2,82 \\
\hline \multirow[t]{2}{*}{-388} & $56-59$ & 2,62 & -447 & $65-73$ & 7,89 & & $314-320$ & 2,35 \\
\hline & $182-186$ & 3,69 & & $70-73$ & 2,21 & & $316-319$ & 2,1 \\
\hline-390 & $40-43$ & 6,91 & & $98-99$ & 2,18 & & $335-341$ & 2,23 \\
\hline-392 & $195-196$ & 2,59 & -450 & $115-119$ & 3,74 & -488 & $388-394$ & 3,47 \\
\hline-394 & $129-130$ & 2,31 & & $155-156$ & 3,07 & & $399-404$ & 3,2 \\
\hline-399 & $114-118$ & 3,47 & & $164-169$ & 4,52 & -489 & $40-45$ & 7,95 \\
\hline-400 & $130-132$ & 3,46 & & $181-185$ & 3,62 & & $64-72$ & 10,93 \\
\hline \multirow[t]{4}{*}{-402} & $76-80$ & 4,23 & -452 & $53-57$ & 2,82 & & $93-98$ & 7,74 \\
\hline & $99-103$ & 3,59 & & $88-92$ & 2,68 & -491 & $155-167$ & 4,32 \\
\hline & $105-107$ & 2,94 & & $190-196$ & 4,03 & & $253-258$ & 2,66 \\
\hline & $231-252$ & 12,94 & -453 & $100-105$ & 5,51 & & $292-297$ & 3,17 \\
\hline-403 & $143-157$ & 5,38 & -460 & $212-217$ & 4 & & $310-316$ & 3,3 \\
\hline-406 & $294-299$ & 3,7 & -461 & $91-94$ & 2,4 & -493 & $117-140$ & 16,83 \\
\hline-407 & $84-91$ & 7,26 & & $99-102$ & 2,89 & -494 & $75-77$ & 3,82 \\
\hline \multirow[t]{2}{*}{-408} & $102-105$ & 3,13 & & $210-214$ & 3,04 & & $78-82$ & 3,69 \\
\hline & $106-108$ & 2,91 & -462 & $331-348$ & 7,32 & -496 & $79-84$ & 3,65 \\
\hline \multirow[t]{3}{*}{-411} & $60-68$ & 3,38 & -463 & $136-149$ & 4,5 & & $87-90$ & 2,85 \\
\hline & $137-144$ & 3,38 & -464 & $200-212$ & 9,55 & & $141-142$ & 2,26 \\
\hline & $147-157$ & 4,17 & -466 & $66-84$ & 10,46 & -497 & $139-145$ & 6,85 \\
\hline-413 & $74-75$ & 2,81 & & $66-70$ & 3,2 & -499 & $57-58$ & 2,8 \\
\hline \multirow[t]{7}{*}{-415} & $54-57$ & 3,7 & & $75-86$ & 8,62 & & $97-98$ & 2,68 \\
\hline & $65-67$ & 2,94 & & $175-177$ & 2,53 & & $107-107$ & 2,63 \\
\hline & $78-83$ & 4,32 & -468 & $78-84$ & 5,31 & -502 & $52-53$ & 2,92 \\
\hline & $88-90$ & 3,01 & -469 & 377 - 391 & 4,95 & -503 & $98-99$ & 2,59 \\
\hline & $89-98$ & 4,96 & -470 & $163-180$ & 12,11 & -509 & dez-14 & 6,15 \\
\hline & $129-135$ & 5,57 & -471 & $61-65$ & 5,09 & & $19-20$ & 3,15 \\
\hline & $153-175$ & 10,89 & & $72-75$ & 4,61 & -516 & $68-69$ & 2,09 \\
\hline-416 & $37-39$ & 3,74 & -473 & $86-90$ & 3,51 & -520 & $371-378$ & 3,11 \\
\hline-419 & $58-61$ & 3,92 & & $125-129$ & 3,29 & -523 & $45-55$ & 8,73 \\
\hline-420 & $101-103$ & 4,87 & & $145-147$ & 2,63 & & $51-63$ & 5,9 \\
\hline-423 & $166-179$ & 10,5 & -474 & $38-39$ & 2,36 & -524 & $291-294$ & 1,79 \\
\hline-424 & $71-74$ & 3,17 & & $82-85$ & 3,53 & -527 & $84-87$ & 7,13 \\
\hline \multirow[t]{2}{*}{-427} & $143-149$ & 3,85 & -476 & $106-113$ & 6,08 & -529 & $117-132$ & 9,84 \\
\hline & $349-356$ & 3,92 & -477 & $110-115$ & 3,73 & & $168-172$ & 3,07 \\
\hline \multirow[t]{2}{*}{-429} & $324-333$ & 5,76 & & $110-112$ & 2,26 & & $264-272$ & 4,79 \\
\hline & $363-365$ & 2,53 & & $182-184$ & 2,35 & & $330-336$ & 4,04 \\
\hline-431 & $57-62$ & 4,86 & -479 & $105-108$ & 2,63 & -530 & $65-69$ & 4,75 \\
\hline-432 & $445-450$ & 6,33 & & $144-150$ & 3,6 & -532 & $89-95$ & 3,56 \\
\hline-434 & $228-237$ & 5,44 & & $260-265$ & 2,8 & -534 & $66-81$ & 12,3 \\
\hline \multirow[t]{2}{*}{-435} & $55-56$ & 3,61 & -480 & $105-105$ & 2,71 & -538 & $322-326$ & 2,72 \\
\hline & $58-58$ & 2,79 & -481 & $131-136$ & 3,91 & -542 & $98-107$ & 3,35 \\
\hline \multirow[t]{2}{*}{-437} & $158-171$ & 5,91 & & $138-145$ & 4,67 & & $335-341$ & 2,67 \\
\hline & $204-211$ & 4,56 & & $142-145$ & 3,14 & -545 & $337-339$ & 2,64 \\
\hline \multirow[t]{2}{*}{-440} & $224-241$ & 7,25 & & $147-154$ & 3,64 & & $437-442$ & 3,72 \\
\hline & $335-350$ & 7,99 & -484 & $39-44$ & 9,41 & -547 & $73-78$ & 3,33 \\
\hline-442 & $42-43$ & 3,62 & -487 & $45-61$ & 4,24 & & $127-133$ & 4,54 \\
\hline
\end{tabular}


Table A.4: Juxta-pleural lung nodules available in the LIDC-IDRI dataset (4 out of 6)

\begin{tabular}{|c|c|c|c|c|c|c|c|c|}
\hline LIDC-IDRI & Slice range & Radius (mm) & LIDC-IDRI & Slice range & Radius (mm) & LIDC-IDRI & Slice range & Radius (mm) \\
\hline \multirow{2}{*}{-549} & $71-74$ & 3,13 & -638 & $111-114$ & 3,22 & & $191-196$ & 4,34 \\
\hline & $122-127$ & 3,4 & -639 & $301-310$ & 4,97 & & $193-198$ & 4,41 \\
\hline-551 & $344-348$ & 2,98 & -641 & $38-40$ & 2,88 & & $209-210$ & 2,18 \\
\hline \multirow[t]{2}{*}{-553} & $375-385$ & 4,44 & & $46-47$ & 2,94 & -687 & $54-56$ & 3,64 \\
\hline & $397-421$ & 8,38 & & $46-56$ & 8,3 & & $79-81$ & 2,78 \\
\hline-554 & $229-232$ & 2,74 & & $71-72$ & 2,96 & -688 & $143-147$ & 2,75 \\
\hline-555 & $147-150$ & 3,27 & & $81-84$ & 4,46 & & $151-163$ & 6,02 \\
\hline \multirow[t]{2}{*}{-559} & $41-43$ & 3,72 & & $93-96$ & 2,73 & & $155-177$ & 10,07 \\
\hline & $67-68$ & 2,59 & -643 & $37-37$ & 2,37 & -695 & $37-39$ & 4,76 \\
\hline-560 & $84-85$ & 3,11 & & $67-69$ & 3,86 & -698 & $333-357$ & 8,54 \\
\hline-563 & $99-100$ & 2,43 & -645 & $108-111$ & 4,33 & & $339-373$ & 13,32 \\
\hline-565 & $92-93$ & 2,85 & -648 & $254-272$ & 7,02 & -699 & $134-137$ & 3,56 \\
\hline-568 & $159-164$ & 5,21 & -649 & $67-70$ & 3,1 & -701 & $188-190$ & 2,56 \\
\hline-569 & $54-56$ & 3,52 & & $102-103$ & 2,38 & & $229-248$ & 9,01 \\
\hline-570 & $110-111$ & 3,66 & & $137-138$ & 2,37 & -702 & $190-211$ & 9,77 \\
\hline \multirow[t]{3}{*}{-571} & $50-53$ & 4,24 & & $200-204$ & 2,98 & & $340-387$ & 17,75 \\
\hline & $51-54$ & 3,64 & & $208-210$ & 2,63 & & $351-356$ & 3,72 \\
\hline & $59-61$ & 3,01 & -650 & $204-209$ & 4,11 & -705 & $89-105$ & 8,09 \\
\hline-572 & $66-77$ & 4,6 & -652 & $125-126$ & 2,97 & & $127-135$ & 5,2 \\
\hline-575 & $114-124$ & 10,97 & -654 & $95-98$ & 2,86 & -708 & $99-101$ & 3,63 \\
\hline \multirow[t]{2}{*}{-577} & $18-19$ & 2,75 & -655 & $134-140$ & 2,92 & -713 & $16-25$ & 13,14 \\
\hline & $22-28$ & 11,27 & & $206-214$ & 3,65 & & $59-59$ & 4,36 \\
\hline-581 & $45-46$ & 2,61 & & $212-220$ & 3,27 & & $82-83$ & 3,09 \\
\hline \multirow[t]{2}{*}{-582} & $499-501$ & 2,33 & & $288-313$ & 8,32 & -714 & $133-147$ & 7,4 \\
\hline & $504-505$ & 2,27 & & $490-500$ & 3,98 & -719 & $28-31$ & 7,38 \\
\hline-584 & $22-26$ & 5,17 & -660 & $93-95$ & 2,86 & -721 & $78-79$ & 3,19 \\
\hline \multirow[t]{3}{*}{-594} & $66-72$ & 3,94 & & $105-109$ & 3,78 & & $85-86$ & 2,72 \\
\hline & $80-98$ & 9,71 & & $106-109$ & 4,24 & -723 & $90-94$ & 3,82 \\
\hline & $86-87$ & 2,49 & & $120-126$ & 6,26 & -733 & $189-197$ & 3,69 \\
\hline \multirow[t]{2}{*}{-599} & $218-221$ & 1,82 & & $135-140$ & 4,78 & & $189-195$ & 3,72 \\
\hline & $392-396$ & 2,15 & & $184-187$ & 2,97 & & $275-277$ & 2,53 \\
\hline-604 & $223-241$ & 4,21 & -661 & $173-202$ & 16,52 & & $293-303$ & 4,32 \\
\hline-605 & $83-88$ & 5,37 & -662 & $152-154$ & 2,86 & -736 & $128-131$ & 2,6 \\
\hline \multirow[t]{2}{*}{-606} & $418-439$ & 5,43 & & $157-159$ & 2,47 & -739 & $265-270$ & 3,65 \\
\hline & $548-552$ & 2,16 & -663 & $292-296$ & 4,12 & -741 & $89-97$ & 7,9 \\
\hline-609 & $179-183$ & 3,46 & -664 & $367-373$ & 3,46 & & $90-91$ & 3,48 \\
\hline-610 & $142-147$ & 6,65 & -666 & $49-51$ & 3,03 & & $125-129$ & 4,17 \\
\hline \multirow[t]{3}{*}{-620} & $89-94$ & 2,83 & & $56-60$ & 4,66 & -743 & $16-18$ & 4,41 \\
\hline & $134-136$ & 2,47 & & $80-82$ & 2,89 & -747 & $78-80$ & 3,22 \\
\hline & $214-215$ & 2,27 & & $112-114$ & 3,21 & -748 & $55-56$ & 2,59 \\
\hline \multirow[t]{2}{*}{-628} & $86-89$ & 2,3 & -669 & $372-379$ & 3,6 & & $74-76$ & 3,42 \\
\hline & $142-143$ & 2,49 & -672 & $175-185$ & 4,35 & -749 & $32-36$ & 6,42 \\
\hline-633 & $50-64$ & 5,05 & -673 & $407-413$ & 2,92 & & $36-37$ & 3,95 \\
\hline-634 & $173-175$ & 2,93 & & $429-433$ & 2,75 & & $46-51$ & 8,57 \\
\hline \multirow[t]{2}{*}{-636} & $190-195$ & 3,52 & -677 & $117-118$ & 2,85 & -751 & $40-50$ & 14,86 \\
\hline & $219-223$ & 3,63 & -681 & $306-328$ & 11,47 & & $79-89$ & 9,12 \\
\hline \multirow[t]{3}{*}{-637} & $197-209$ & 4,27 & -686 & $88-89$ & 2,18 & & $84-92$ & 14,29 \\
\hline & $308-314$ & 3,24 & & $97-100$ & 3,6 & -753 & $56-63$ & 7,41 \\
\hline & $407-414$ & 3,2 & & $149-156$ & 5,97 & -754 & $97-99$ & 2,66 \\
\hline
\end{tabular}


Table A.5: Juxta-pleural lung nodules available in the LIDC-IDRI dataset (5 out of 6)

\begin{tabular}{|c|c|c|c|c|c|c|c|c|}
\hline LIDC-IDRI & Slice range & Radius (mm) & LIDC-IDRI & Slice range & Radius (mm) & LIDC-IDRI & Slice range & Radius (mm) \\
\hline & $126-130$ & 3,45 & -807 & $237-240$ & 3,53 & & $63-72$ & 11,64 \\
\hline & $180-182$ & 2,52 & -809 & $141-146$ & 4,59 & -863 & $188-199$ & 4,58 \\
\hline-756 & $326-344$ & 9,82 & -810 & $397-412$ & 5,76 & -864 & $129-132$ & 3,55 \\
\hline-757 & $420-428$ & 4,05 & -813 & $73-74$ & 2,24 & -866 & $53-56$ & 3,68 \\
\hline \multirow[t]{2}{*}{-761} & $131-135$ & 2,85 & -819 & $294-322$ & 12,47 & & $105-108$ & 3,88 \\
\hline & $137-144$ & 4,52 & -820 & $132-133$ & 2,31 & & $152-153$ & 2,16 \\
\hline \multirow[t]{5}{*}{-762} & $59-62$ & 2,76 & & $214-217$ & 3,1 & & $195-197$ & 2,58 \\
\hline & $60-66$ & 3,39 & -821 & $369-374$ & 2,62 & -868 & $48-50$ & 3,29 \\
\hline & $74-77$ & 2,9 & & $369-382$ & 4,62 & -871 & $174-179$ & 3,94 \\
\hline & $76-80$ & 3,15 & -829 & $131-169$ & 14,18 & & $189-192$ & 3,33 \\
\hline & $88-91$ & 3,53 & -831 & $412-415$ & 3,18 & & $191-194$ & 3,4 \\
\hline \multirow{3}{*}{-765} & $64-70$ & 7,11 & -832 & $49-49$ & 1,93 & & $198-200$ & 2,56 \\
\hline & $71-73$ & 5,59 & & $60-61$ & 3,17 & -874 & $311-317$ & 2,97 \\
\hline & $90-95$ & 5,54 & -836 & $36-37$ & 2,74 & -879 & $136-140$ & 3,22 \\
\hline \multirow[t]{4}{*}{-770} & $123-131$ & 4,64 & -837 & $366-392$ & 9,3 & & $221-222$ & 3,08 \\
\hline & $183-188$ & 3,58 & -838 & $118-124$ & 3,77 & -880 & $123-127$ & 4,07 \\
\hline & $220-223$ & 2,89 & & $172-179$ & 3,67 & & $173-175$ & 2,49 \\
\hline & $241-252$ & 5,23 & & $258-261$ & 2,3 & -882 & $135-140$ & 4,45 \\
\hline-772 & $112-117$ & 9,88 & -842 & $90-91$ & 2,56 & -883 & $162-169$ & 5,93 \\
\hline \multirow[t]{7}{*}{-775} & $77-82$ & 3,86 & -843 & $161-163$ & 2,5 & & $169-174$ & 4,07 \\
\hline & $92-94$ & 2,35 & & $193-201$ & 3,78 & & $180-185$ & 4,73 \\
\hline & $116-123$ & 4,83 & & $212-222$ & 5,24 & -890 & $50-70$ & 10,85 \\
\hline & $123-128$ & 3,54 & & $225-232$ & 3,38 & -894 & $64-66$ & 2,89 \\
\hline & $168-174$ & 5,16 & & $310-314$ & 2,5 & & $86-91$ & 4,48 \\
\hline & $223-252$ & 12,56 & -846 & $115-115$ & 2,65 & & $113-115$ & 2,72 \\
\hline & $248-252$ & 2,97 & -849 & $53-66$ & 3,82 & & $184-187$ & 3,8 \\
\hline \multirow[t]{3}{*}{-776} & $111-113$ & 2,54 & & $122-130$ & 4,12 & -896 & $29-31$ & 3,66 \\
\hline & $187-190$ & 2,86 & & $246-251$ & 3,14 & & $81-87$ & 6,26 \\
\hline & $197-204$ & 5,4 & & $259-265$ & 3,05 & -899 & $82-84$ & 3,53 \\
\hline \multirow[t]{2}{*}{-780} & $219-252$ & 11,74 & & $297-313$ & 6,8 & -902 & $87-101$ & 6,6 \\
\hline & $254-259$ & 3,32 & & $299-310$ & 5,74 & & $244-247$ & 2,57 \\
\hline-781 & $303-310$ & 3,5 & -850 & $43-56$ & 9,22 & -905 & $158-169$ & 8,24 \\
\hline \multirow[t]{4}{*}{-785} & $99-108$ & 7,06 & & $90-91$ & 1,99 & -906 & $29-43$ & 9,36 \\
\hline & $136-154$ & 12,93 & -854 & $125-131$ & 3,32 & & $50-52$ & 3,02 \\
\hline & $160-161$ & 2,76 & & $126-136$ & 4,79 & & $51-57$ & 4,83 \\
\hline & $168-172$ & 3,51 & & $167-172$ & 3,63 & -907 & $175-177$ & 2,43 \\
\hline-787 & $72-74$ & 3,29 & & $236-240$ & 2,48 & & $209-212$ & 2,76 \\
\hline \multirow[t]{2}{*}{-790} & $93-100$ & 6,4 & -855 & $44-45$ & 3,02 & -909 & $169-176$ & 4,27 \\
\hline & $121-155$ & 15,1 & & $60-61$ & 3,26 & -911 & $37-45$ & 8,26 \\
\hline \multirow[t]{2}{*}{-801} & $229-238$ & 3,56 & & $74-75$ & 3,18 & -914 & $102-104$ & 2,49 \\
\hline & $399-403$ & 3,52 & -857 & $107-115$ & 4,18 & -916 & $42-43$ & 2,81 \\
\hline-805 & $196-201$ & 4,9 & -858 & $146-150$ & 2,76 & & $62-63$ & 2,77 \\
\hline \multirow[t]{7}{*}{-806} & $112-116$ & 2,86 & & $165-175$ & 5,74 & -920 & $319-340$ & 11,49 \\
\hline & $247-252$ & 3,34 & & $216-220$ & 3,25 & -923 & $219-226$ & 4,07 \\
\hline & $261-267$ & 5,05 & & $283-287$ & 2,69 & -925 & $129-141$ & 7,07 \\
\hline & $296-302$ & 4,44 & & $286-298$ & 6,83 & -928 & $149-152$ & 2,39 \\
\hline & $303-311$ & 4,12 & -859 & $87-91$ & 3 & -932 & $20-23$ & 3,88 \\
\hline & $328-337$ & 5,01 & -860 & $102-106$ & 5,17 & -935 & $48-50$ & 3,27 \\
\hline & $428-435$ & 3,67 & -861 & $51-53$ & 4,06 & & $50-50$ & 2,46 \\
\hline
\end{tabular}


Table A.6: Juxta-pleural lung nodules available in the LIDC-IDRI dataset (6 out of 6)

\begin{tabular}{|c|c|c|}
\hline LIDC-IDRI & Slice range & Radius (mm) \\
\hline & $56-57$ & 4,14 \\
\hline & $58-58$ & 2,55 \\
\hline & $67-69$ & 5,79 \\
\hline \multirow{2}{*}{-939} & $191-194$ & 2,55 \\
\hline & $368-390$ & 9,75 \\
\hline-941 & $236-244$ & 5,33 \\
\hline-943 & $151-156$ & 3,2 \\
\hline-944 & $53-55$ & 3,9 \\
\hline-946 & $46-49$ & 6,59 \\
\hline-950 & $213-214$ & 2,26 \\
\hline \multirow[t]{2}{*}{-956} & $130-131$ & 2,71 \\
\hline & $150-154$ & 3,67 \\
\hline-957 & $36-37$ & 4,22 \\
\hline-958 & $22-26$ & 5,27 \\
\hline \multirow[t]{3}{*}{-962} & $17-17$ & 2,99 \\
\hline & $18-18$ & 1,98 \\
\hline & $59-61$ & 5,27 \\
\hline-963 & $213-220$ & 3,5 \\
\hline \multirow[t]{2}{*}{-968} & $109-130$ & 11,3 \\
\hline & $186-189$ & 2,61 \\
\hline-971 & $50-57$ & 3,94 \\
\hline \multirow[t]{2}{*}{-973} & $127-133$ & 5,87 \\
\hline & $144-150$ & 6,26 \\
\hline \multirow[t]{2}{*}{-976} & $45-63$ & 15,1 \\
\hline & $49-50$ & 2,86 \\
\hline-977 & $35-36$ & 3,18 \\
\hline-982 & $480-493$ & 6,62 \\
\hline \multirow[t]{3}{*}{-985} & $145-154$ & 3,93 \\
\hline & $163-167$ & 2,21 \\
\hline & $342-347$ & 2,38 \\
\hline-987 & $190-195$ & 3,42 \\
\hline-991 & $160-162$ & 2,82 \\
\hline \multirow[t]{3}{*}{-994} & $171-179$ & 4,15 \\
\hline & $198-206$ & 5,74 \\
\hline & $433-449$ & 7,82 \\
\hline-997 & $183-186$ & 2,33 \\
\hline \multirow[t]{7}{*}{-998} & $32-34$ & 4,11 \\
\hline & $36-39$ & 5,32 \\
\hline & $50-52$ & 5,82 \\
\hline & $57-62$ & 7,22 \\
\hline & $65-66$ & 3,59 \\
\hline & $68-69$ & 4,76 \\
\hline & $70-71$ & 3,85 \\
\hline \multirow{3}{*}{-1003} & $55-59$ & 5,09 \\
\hline & $67-78$ & 8,93 \\
\hline & $86-99$ & 12,35 \\
\hline \multirow[t]{2}{*}{-1004} & $368-395$ & 11,95 \\
\hline & $403-416$ & 5,3 \\
\hline \multirow{2}{*}{-1005} & $115-121$ & 4,16 \\
\hline & $180-182$ & 2,69 \\
\hline-1012 & $61-64$ & 4,88 \\
\hline
\end{tabular}




\section{References}

Ahn, M. I., Gleeson, T. G., Chan, I. H., et al. Perifissural Nodules Seen at CT Screening for Lung Cancer 1. Radiology, 2010, 254(3):949-956. doi: 10.1148/radiol.09090031. URL http: //pubs.rsna.org/doi/abs/10.1148/radiol.09090031.

Ajanki, A. 2007, kNN Classification. URLhttps://commons.wikimedia.org/wiki/Fil e\{으 3AKnnClassification.svg.

Alilou, M., Kovalev, V., Snezhko, E., et al. A comprehensive framework for automatic detection of pulmonary nodules in lung CT images. Image Analysis \& Stereology, 2014, 33(1):13. doi: 10.5566/ias.v33.p13-27. URL http://www.ias-iss.org/ojs/IAS/article/view/ 1081 .

ANODE09. 2009, Automatic Nodule Detection 2009. URL http://anode09.grand-chall enge.org/,

Armato, S. G., McLennan, G., Bidaut, L., et al. The Lung Image Database Consortium (LIDC) and Image Database Resource Initiative (IDRI): A Completed Reference Database of Lung Nodules on CT Scans. Medical Physics, 2011, 38(2):915. doi: 10.1118/1.3528204. URL http://scit ation.aip.org/content/aapm/journal/medphys/38/2/10.1118/1.3528204.

Armato III, S. G., McLennan, G., McNitt-Gray, M. F., et al. Lung Image Database Consortium: Developing a Resource for the Medical Imaging Research Community1. Radiology, 2004, 232 (3):739-748. doi: 10.1148/radiol.2323032035. URL/http://pubs.rsna.org/doi/abs/ 10.1148 /radiol.2323032035.

Bach, P. B., Mirkin, J. N., Oliver, T. K., et al. Benefits and Harms of CT Screening for Lung Cancer. JAMA, 2012, 307(22):2418. doi: 10.1001/jama.2012.5521. URL http: / / jama . jam anetwork.com/article.aspx?doi=10.1001/jama.2012.5521.

Badura, P. and Pietka, E. Soft computing approach to 3D lung nodule segmentation in CT. Computers in Biology and Medicine, 2014, 53:230-243. doi: 10.1016/j.compbiomed.2014.08.005. URL http://linkinghub.elsevier.com/retrieve/pii/s0010482514002078.

Brown, M. S., Lo, P., Goldin, J. G., et al. Toward clinically usable CAD for lung cancer screening with computed tomography. European Radiology, 2014, 24(11):27192728. doi: 10.1007/s00330-014-3329-0. URL http://link.springer.com/10.1007/ s00330-014-3329-0.

Camarlinghi, N., Gori, I., Retico, A., et al. Combination of computer-aided detection algorithms for automatic lung nodule identification. International Journal of Computer Assisted Radiology and Surgery, 2012, 7(3):455-464. doi: 10.1007/s11548-011-0637-6. URL http://link .s pringer.com/10.1007/s11548-011-0637-6. 
Cascio, D., Magro, R., Fauci, F., et al. Automatic detection of lung nodules in CT datasets based on stable 3D mass-spring models. Computers in Biology and Medicine, 2012, 42(11):10981109. doi: 10.1016/j.compbiomed.2012.09.002. URL http:// linkinghub.elsevier. com/retrieve/pii/s0010482512001400.

Castellino, R. A. Computer aided detection (CAD): an overview. Cancer Imaging, 2005, 5(1):1719. doi: 10.1102/1470-7330.2005.0018. URL http://www. cancerimaging.org/2005/ $5 / 1 / 0018 /$.

Chan, T. F. and Vese, L. A. Active contours without edges. IEEE Transactions on Image Processing, 2001, 10(2):266-277. doi: 10.1109/83.902291.

Chang, C.-C. and Lin, C.-J. \{LIBSVM \}: A library for support vector machines. ACM Transactions on Intelligent Systems and Technology, 2011, 2(3):27:1—-27:27.

Choi, W.-J. and Choi, T.-S. Automated Pulmonary Nodule Detection System in Computed Tomography Images: A Hierarchical Block Classification Approach. Entropy, 2013, 15(2):507-523. doi: 10.3390/e15020507. URL http://www.mdpi.com/1099-4300/15/2/507/.

Choi, W.-J. and Choi, T.-S. Automated pulmonary nodule detection based on three-dimensional shape-based feature descriptor. Computer Methods and Programs in Biomedicine, 2014, 113 (1):37-54. doi: 10.1016/j.cmpb.2013.08.015. URL http://linkinghub.elsevier.co $\mathrm{m} /$ retrieve/pii/s0169260713002940.

Davidian, D. 1995, Feed-forward neural network. URL http://www.google.com/patents /US5438646.

De Angelis, R., Sant, M., Coleman, M. P., et al. Cancer survival in Europe 1999-2007 by country and age: results of EUROCARE-5-a population-based study. The Lancet Oncology, 2014, 15 (1):23-34. doi: 10.1016/S1470-2045(13)70546-1. URL http:// linkinghub.elsevier. com/retrieve/pii/S1470204513705461.

de Carvalho Filho, A. O., de Sampaio, W. B., Silva, A. C., et al. Automatic detection of solitary lung nodules using quality threshold clustering, genetic algorithm and diversity index. Artificial Intelligence in Medicine, 2014, 60(3):165-177. doi: 10.1016/j.artmed.2013.11.002. URL ht tp://linkinghub.elsevier.com/retrieve/pii/s0933365713001541.

De Nunzio, G., Massafra, A., Cataldo, R., et al. Approaches to juxta-pleural nodule detection in CT images within the MAGIC-5 Collaboration. Nuclear Instruments and Methods in Physics Research Section A: Accelerators, Spectrometers, Detectors and Associated Equipment, 2011, 648:S103-S106. doi: 10.1016/j.nima.2010.12.082. URL http://linkinghub.elsevie r.com/retrieve/pii/s0168900210028238.

Dice, L. Measures of the amount of ecologic association between species. Ecology, 1945, 26: 297-302. doi: doi:10.2307/1932409.

El-Baz, A., Beache, G. M., Gimel'farb, G., et al. Computer-Aided Diagnosis Systems for Lung Cancer: Challenges and Methodologies. International Journal of Biomedical Imaging, 2013, 2013:1-46. doi: 10.1155/2013/942353. URL http://www.hindawi.com/journals/i jbi/2013/942353/. 
Flach, P., Hernández-Orallo, J., and Ferri, C. A coherent interpretation of auc as a measure of aggregated classification performance. International Conference on Machine Learning, 2011, (June 2011):657-664. doi: 10.1145/347090.347126. URL http://www . icml-2011.org/ papers/385\{_\}icmlpaper.pdf.

Getreuer, P. Chan-Vese Segmentation. Image Processing On Line, 2012, 2:214-224. doi: 10. 5201/ipol.2012.g-cv. URL http://www.ipol.im/pub/art/2012/g-cv/.

Glass, G. V. and Hopkins, K. D. Statistical Methods in Education and Psychology. Statistical Methods in Education and Psychology. Allyn and Bacon, 1996, 3 edition. URL https://bo oks.google.pt/books?id=SFmdAAAAMAAJ.

Goldman, L. W. Principles of CT: Multislice CT. Journal of Nuclear Medicine Technology, 2008, 36(2):57-68. doi: 10.2967/jnmt.107.044826. URL http://tech.snmjournals.org/c gi/doi/10.2967/jnmt.107.044826.

Golosio, B., Masala, G. L., Piccioli, A., et al. A novel multithreshold method for nodule detection in lung CT. Medical Physics, 2009, 36(8):3607. doi: 10.1118/1.3160107. URL http://scit ation.aip.org/content/aapm/journal/medphys/36/8/10.1118/1.3160107,

Gray, H., Gray, H., and Lewis, W. H. Anatomy of the human body. Lea \& Febiger,, Philadelphia,, 1918, 20th edition. doi: 10.5962/bhl.title.20311. URL http://www.biodiversitylibr ary.org/bibliography/20311.

Guo, W. and Li, Q. High performance lung nodule detection schemes in CT using local and global information. Medical Physics, 2012, 39(8):5157. doi: 10.1118/1.4737109. URL http://scitation.aip.org/content/aapm/journal/medphys/39/8/10. $1118 / 1.4737109$.

Han, H., Li, L., Han, F., et al. Fast and Adaptive Detection of Pulmonary Nodules in Thoracic CT Images Using a Hierarchical Vector Quantization Scheme. IEEE Journal of Biomedical and Health Informatics, 2015, 19(2):648-659. doi: 10.1109/JBHI.2014.2328870. URL http: //ieeexplore.ieee.org/lpdocs/epic03/wrapper.htm?arnumber $=6825802$.

Hearst, M., Dumais, S., Osman, E., et al. Support vector machines. IEEE Intelligent Systems, 1998, 13(4):18-28. doi: 10.1109/5254.708428. URL/http://ieeexplore.ieee.org/lp docs/epic03/wrapper.htm?arnumber $=708428$.

Holmes, E. J., Forrest-Hay, A. C., and Misra, R. R. Interpretation of Emergency Head CT. Cambridge University Press, 2008, Cambridge. doi: 10.1017/CBO9780511544804. URL https://books.google.pt/books?id=TYg6QRSnvw0Chttp://ebooks.cam bridge.org/ref/id/CB09780511544804.

Jacobs, C., van Rikxoort, E. M., Twellmann, T., et al. Automatic detection of subsolid pulmonary nodules in thoracic computed tomography images. Medical Image Analysis, 2014, 18(2):374-384. doi: 10.1016/j.media.2013.12.001. URL http://dx.doi.org/10.1016/j.media.2013.12.001http://linkinghub .elsevier.com/retrieve/pii/s1361841513001722.

Kong, H., Akakin, H. C., and Sarma, S. E. A generalized laplacian of gaussian filter for blob detection and its applications. IEEE Transactions on Cybernetics, 2013, 43(6):1719-1733. doi: 10.1109/TSMCB.2012.2228639. 
Li, Q., Sone, S., and Doi, K. Selective enhancement filters for nodules, vessels, and airway walls in two- and three-dimensional CT scans. Medical Physics, 2003, 30(8):2040. doi: 10.1118/1.1581411. URL http://scitation.aip.org/content/aapm/journal/m edphys/30/8/10.1118/1.1581411.

Lopez Torres, E., Fiorina, E., Pennazio, F., et al. Large scale validation of the M5L lung CAD on heterogeneous CT datasets. Medical Physics, 2015, 42(4):1477-1489. doi: 10.1118/ 1.4907970. URL http://www.ncbi.nlm.nih.gov/pubmed/25832038http://scit ation.aip.org/content/aapm/journal/medphys/42/4/10.1118/1.4907970.

Marr, D. and Hildreth, E. Theory of Edge Detection. Proceedings of the Royal Society of London B: Biological Sciences, 1980, 207(1167):187-217. doi: 10.1098/rspb.1980.0020. URL http: //rspb.royalsocietypublishing.org/content/207/1167/187.

Messay, T., Hardie, R. C., and Rogers, S. K. A new computationally efficient CAD system for pulmonary nodule detection in CT imagery. Medical Image Analysis, 2010, 14(3): 390-406. doi: 10.1016/j.media.2010.02.004. URL http://dx.doi.org/10.1016/ j.media.2010.02.004http://linkinghub.elsevier.com/retrieve/pii/s 1361841510000198 .

Murphy, K., van Ginneken, B., Schilham, A., et al. A large-scale evaluation of automatic pulmonary nodule detection in chest CT using local image features and k-nearest-neighbour classification. Medical Image Analysis, 2009, 13(5):757-770. doi: 10.1016/j.media.2009.07. 001. URL http://dx.doi.org/10.1016/j.media.2009.07.001http://linkin ghub.elsevier.com/retrieve/pii/S1361841509000516.

NIH. 2015, What is cancer? URL/http://www. cancer.gov/about-cancer/what-is-c ancer.

Novo, J., Rouco, J., Mendonça, A., et al. Reliable lung segmentation methodology by including juxtapleural nodules, volume 8815 of Lecture Notes in Computer Science. Springer International Publishing, 2014, Cham. doi: 10.1007/978-3-319-11755-3. URL http://link.spr inger.com/10.1007/978-3-319-11755-3

Novo, J., Goncalves, L., Mendonça, A. M., et al. 3D lung nodule candidate detection in multiple scales. In 2015 14th IAPR International Conference on Machine Vision Applications (MVA), pages 61-64. 2015, IEEE. doi: 10.1109/MVA.2015.7153133. URL http://ieeexplore.i eee.org/lpdocs/epic03/wrapper.htm?arnumber=7153133.

Okada, K., Ramesh, V., Krishnan, A., et al. Robust Pulmonary Nodule Segmentation in CT: Improving Performance for Juxtapleural Cases. In Medical image computing and computerassisted intervention: MICCAI, International Conference on Medical Image Computing and Computer-Assisted Intervention, volume 8, 2005. pages 781-789. doi: 10.1007/11566489_ 96. URL http://www.ncbi.nlm.nih.gov/pubmed/16686031http://link.sprin ger.com/10.1007/11566489\{_\}96.

OpenStaxCollege-Anatomy\&Physiology. 2013, 2313 The Lung Pleurea. URL http://images.medicinenet.com/images/appictures/pleurisy-pleur itis-s4-how-pleura-works.jpg.

Opfer, R. and Wiemker, R. Performance analysis for computer-aided lung nodule detection on LIDC data. In Jiang, Y. and Sahiner, B., editors, Proceedings of SPIE, volume 6515, 2007, 
pages 65151C-65151C-9. doi: 10.1117/12.708210. URL http://proceedings.spiedi gitallibrary.org/proceeding.aspx?articleid=1336879.

Otsu, N. A Threshold Selection Method from Gray-Level Histograms. IEEE Transactions on Systems, Man, and Cybernetics, 1979, 9(1):62-66. doi: 10.1109/TSMC.1979. 4310076. URL http://ieeexplore.ieee.org/lpdocs/epic03/wrapper.htm?ar number $=4310076$.

Pedregosa, F., Varoquaux, G., Gramfort, A., et al. Scikit-learn: Machine Learning in $\{\mathrm{P}\}$ ython. Journal of Machine Learning Research, 2011, 12:2825-2830.

Platt, J. Sequential minimal optimization: A fast algorithm for training support vector machines. Advances in Kernel MethodsSupport Vector Learning, 1998, 208:1-21. doi: 10.1.1. 43.4376. URL http://citeseerx.ist.psu.edu/viewdoc/download?doi=10.1. $1.55 .560\{\&\}$ rep $=r e p 1\{\&\}$ type $=p d f$.

$\mathrm{Pu}$, J., Zheng, B., Leader, J. K., et al. An automated CT based lung nodule detection scheme using geometric analysis of signed distance field. Medical Physics, 2008, 35(8):3453. doi: 10.1118/1.2948349. URL http://link.aip.org/link/MPHYA6/v35/i8/p3453/ s1\{\&\}Agg=doihttp://scitation.aip.org/content/aapm/journal/medphys /35/8/10.1118/1.2948349.

Retico, A., Delogu, P., Fantacci, M., et al. Lung nodule detection in low-dose and thin-slice computed tomography. Computers in Biology and Medicine, 2008, 38(4):525-534. doi: 10.1016/j.compbiomed.2008.02.001. URL http://linkinghub.elsevier.com/retr ieve/pii/s0010482508000206.

Retico, A., Fantacci, M., Gori, I., et al. Pleural nodule identification in low-dose and thin-slice lung computed tomography. Computers in Biology and Medicine, 2009, 39(12):1137-1144. doi: 10.1016/j.compbiomed.2009.10.005. URL http: //www.ncbi.nlm.nih.gov/pubmed/19883906http://linkinghub.elsevie r.com/retrieve/pii/s0010482509001814

Ridler, T. and Calvard, S. Picture Thresholding Using an Iterative Selection Method. IEEE Transactions on Systems, Man, and Cybernetics, 1978, 8(8):630-632. doi: 10.1109/TSMC.1978. 4310039. URL http://ieeexplore.ieee.org/lpdocs/epic03/wrapper.htm?ar number $=4310039$.

Setio, A. A. A., Jacobs, C., Gelderblom, J., et al. Automatic detection of large pulmonary solid nodules in thoracic CT images. Medical Physics, 2015, 42(10):5642-5653. doi: 10.1118/1.4929562. URL http://scitation.aip.org/content/aapm/journal/m edphys/42/10/10.1118/1.4929562.

Siegel, R. L., Miller, K. D., and Jemal, A. Cancer statistics, 2015. CA: A Cancer Journal for Clinicians, 2015, 65(1):5-29. doi: 10.3322/caac.21254. URL http://doi.wiley.com/ $10.3322 / \mathrm{caac} .21254$.

Smola, A. and Vishwanathan, S. Introduction to machine learning. Cambridge University Press, 2014, 1107:105-128. URL http://alex.smola.org/drafts/thebook.pdf

Soret, M., Bacharach, S. L., and Buvat, I. Partial-Volume Effect in PET Tumor Imaging. Journal of Nuclear Medicine, 2007, 48(6):932-945. doi: 10.2967/jnumed.106.035774. URL http: //jnm.snmjournals.org/cgi/doi/10.2967/jnumed.106.035774. 
Sun, S., Guo, Y., Guan, Y., et al. Juxta-Vascular Nodule Segmentation Based on Flow Entropy and Geodesic Distance. IEEE Journal of Biomedical and Health Informatics, 2014, 18(4): 1355-1362. doi: 10.1109/JBHI.2014.2303511. URL http://ieeexplore.ieee.org/lp docs/epic03/wrapper.htm?arnumber $=6728607$.

Swensen, S. J., Jett, J. R., Hartman, T. E., et al. Lung Cancer Screening with CT: Mayo Clinic Experience1. Radiology, 2003, 226(3):756-761. doi: 10.1148/radiol.2263020036. URL http://dx.doi.org/10.1148/radiol.2263020036http://pubs.rsna.or g/doi/abs/10.1148/radiol.2263020036.

Tan, M., Deklerck, R., Jansen, B., et al. A novel computer-aided lung nodule detection system for CT images. Medical Physics, 2011, 38(10):5630. doi: 10.1118/1. 3633941. URL http://scitation.aip.org/content/aapm/journal/medphys /38/10/10.1118/1.3633941.

Tan, M., Deklerck, R., Cornelis, J., et al. Phased searching with NEAT in a Time-Scaled Framework: Experiments on a computer-aided detection system for lung nodules. Artificial Intelligence in Medicine, 2013, 59(3):157-167. doi: 10.1016/j.artmed.2013.07. 002. URL http://dx.doi.org/10.1016/j.artmed.2013.07.002http://linkin ghub.elsevier.com/retrieve/pii/s0933365713000985.

Taşc1, E. and Uğur, A. Shape and Texture Based Novel Features for Automated Juxtapleural Nodule Detection in Lung CTs. Journal of Medical Systems, 2015, 39(5):46. doi: 10.1007/ s10916-015-0231-5. URL http://www.ncbi.nlm.nih.gov/pubmed/25732079htt $\mathrm{p}$ ://link.springer.com/10.1007/s10916-015-0231-5.

Teramoto, A. and Fujita, H. Fast lung nodule detection in chest CT images using cylindrical nodule-enhancement filter. International Journal of Computer Assisted Radiology and Surgery, 2013, 8(2):193-205. doi: 10.1007/s11548-012-0767-5. URL http://link.springer.co $\mathrm{m} / 10.1007 / \mathrm{s} 11548-012-0767-5$.

Terzopoulos, D. and Fleischer, K. Deformable models. The Visual Computer, 1988, 4(6): 306-331. doi: 10.1007/BF01908877. URL http://link.springer.com/10.1007/BF 01908877

TheCancerImagingArchiveTeam. Data From LIDC-IDRI. doi: 10.7937/K9/TCIA.2015.LO9Q L9SX. URL https://wiki.cancerimagingarchive.net/display/Public/LID C-IDRI.

Valente, I. R. S., Cortez, P. C., Neto, E. C., et al. Automatic 3D pulmonary nodule detection in CT images: A survey. Computer Methods and Programs in Biomedicine, 2016, 124:91-107. doi: 10.1016/j.cmpb.2015.10.006. URL http:// linkinghub.elsevier.com/retrieve/p ii/S0169260715300298.

van Ginneken, B., Armato, S. G., de Hoop, B., et al. Comparing and combining algorithms for computer-aided detection of pulmonary nodules in computed tomography scans: The ANODE09 study. Medical Image Analysis, 2010, 14(6):707-722. doi: 10.1016/ j.media.2010.05.005. URL http://linkinghub.elsevier.com/retrieve/pii/S 1361841510000587 .

van Ginneken, B., Setio, A. A. A., Jacobs, C., et al. Off-the-shelf convolutional neural network features for pulmonary nodule detection in computed tomography scans. In 2015 IEEE 12th 
International Symposium on Biomedical Imaging (ISBI), pages 286-289. 2015, IEEE. doi: 10.1109/ISBI.2015.7163869. URL http://ieeexplore.ieee.org/lpdocs/epic03/ wrapper.htm?arnumber $=7163869$.

WolframMathematica. Feedforward Neural Networks. URL http://reference.wolfram. com/applications/neuralnetworks/NeuralNetworkTheory/2.5.1.html.

WorldHealthOrganization. 2015, WHO I Cancer. URL http://www . who. int/mediacentr e/factsheets/fs297/en/.

Wu, X., Kumar, V., Ross Quinlan, J., et al. Top 10 algorithms in data mining. Knowledge and Information Systems, 2008, 14(1):1-37. doi: 10.1007/s10115-007-0114-2. URL http://li nk.springer.com/10.1007/s10115-007-0114-2

Yu-Jen Chen, Y.-J., Hua, K.-L., Hsu, C.-H., et al. Computer-aided classification of lung nodules on computed tomography images via deep learning technique. OncoTargets and Therapy, 2015, page 2015. doi: 10.2147/OTT.S80733. URL http://www.dovepress.com/computer-aided-classification-of-lung-nod ules-on-computed-tomography-i-peer-reviewed-article-OTT. 